\title{
SEMIDIRECT PRODUCTS OF C*-QUANTUM GROUPS: MULTIPLICATIVE UNITARIES APPROACH
}

\author{
RALF MEYER, SUTANU ROY, AND STANISŁAW LECH WORONOWICZ
}

\begin{abstract}
C}^{*}$-quantum groups with projection are the noncommutative analogues of semidirect products of groups. Radford's Theorem about Hopf algebras with projection suggests that any $\mathrm{C}^{*}$-quantum group with projection decomposes uniquely into an ordinary $\mathrm{C}^{*}$-quantum group and a "braided" $\mathrm{C}^{*}$-quantum group. We establish this on the level of manageable multiplicative unitaries.
\end{abstract}

\section{INTRODUCTION}

Many important Lie groups like the Poincaré group or the group of motions of Euclidean space are defined as semidirect products of smaller building blocks. What is the quantum group analogue of a semidirect product? Such a notion should be useful to understand quantum deformations of semidirect products.

For a semidirect product of groups, we need two groups $G$ and $H$ and an action of $G$ on $H$ by group automorphisms. Since non-commutative quantum groups cannot act on other quantum groups by automorphisms, we need a different point of view: semidirect product groups are the same as groups with a projection. A semidirect product of groups $G \ltimes H$ comes with a canonical group homomorphism

$$
p: G \ltimes H \rightarrow G \ltimes H, \quad(g, h) \mapsto\left(g, 1_{H}\right),
$$

which is idempotent, that is, $p^{2}=p$. Its kernel and image are $H \subseteq G \ltimes H$ and $G \subseteq G \ltimes H$, respectively. The conjugation action of $G$ on $H$ needed for a semidirect product is the restriction of the conjugation action of $G \ltimes H$ on itself. Therefore, an idempotent group homomorphism $p: K \rightarrow K$ on a group $K$ is equivalent to a semidirect product decomposition of $K$.

Now consider a quantum group with a projection, that is, with an idempotent quantum group endomorphism. What corresponds to the building blocks $G$ and $H$ in a semidirect product of groups? If "quantum group" means "Hopf algebra," then a theorem by Radford [15] answers this question. Here we consider $\mathrm{C}^{*}$-quantum groups, meaning $\mathrm{C}^{*}$-bialgebras coming from manageable multiplicative unitaries (see $[21,28]$ ). More precisely, we work on the level of the multiplicative unitaries themselves to avoid analytical difficulties.

Let us first recall Radford's Theorem. It splits a Hopf algebra $C$ with a projection $p: C \rightarrow C$ into two pieces $A$ and $B$. The "image" of the projection $A$ is a Hopf algebra as well. The "kernel" of the projection $B$ is only a Hopf algebra in a certain braided monoidal category, namely, the category of Yetter-Drinfeld modules

2000 Mathematics Subject Classification. 46L89 (81R50 18D10).

Key words and phrases. quantum group, braided quantum group, semidirect product, bosonisation, multiplicative unitary, braided multiplicative unitary, quantum E(2) group.

Supported by the German Research Foundation (Deutsche Forschungsgemeinschaft (DFG)) through the Research Training Group 1493. The second author was also supported by a FieldsOntario postdoctoral fellowship. The third author was partially supported by the Alexander von Humboldt-Stiftung and the National Science Center (NCN), grant 2015/17/B/ST1/00085. 
over $A$. The tensor product of two Yetter-Drinfeld algebras is again a YetterDrinfeld algebra, for the diagonal Yetter-Drinfeld module structure and a certain deformed multiplication. The comultiplication on $B$ is a homomorphism to the deformed tensor product $B \otimes B$.

Radford's Theorem contains two constructions. One puts together $A$ and $B$ into their "semidirect product" $C$ and describes the projection $p$ on $C$. The other splits $C$ into the two factors $A$ and $B$, with the Hopf algebra structure on $A$ and the $A$-Yetter-Drinfeld algebra and braided Hopf algebra structure on $B$. The first construction is called "bosonisation" by Majid [8. The analogue of this construction for $\mathrm{C}^{*}$-quantum groups is described in 12 , except for the projection that we expect on this semidirect product. In particular, the appropriate analogues of YetterDrinfeld algebras and their deformed tensor product $\otimes$ are described in 12 for arbitrary $\mathrm{C}^{*}$-quantum groups. For regular $\mathrm{C}^{*}$-quantum groups with Haar weights, this is already done by Nest and Voigt [13].

The "projections" on $\mathrm{C}^{*}$-quantum groups that we use are morphisms as introduced in [10,14. That is, a quantum group morphism from $\left(C, \Delta_{C}\right)$ to $\left(A, \Delta_{A}\right)$ is a bicharacter in $\mathcal{U} \mathcal{M}(\hat{C} \otimes A)$. Several equivalent descriptions of such morphisms are given in [10, including functors between the categories of $\mathrm{C}^{*}$-algebra coactions that preserve the underlying $\mathrm{C}^{*}$-algebra, and Hopf ${ }^{*}$-homomorphisms between the associated universal quantum groups. These are more general than Hopf *-homomorphisms between the reduced quantum group $\mathrm{C}^{*}$-algebras.

Thus a $\mathrm{C}^{*}$-quantum group with projection consists of a $\mathrm{C}^{*}$-quantum group $\left(C, \Delta_{C}\right)$ with a unitary multiplier $\mathrm{P} \in \mathcal{U} \mathcal{M}(\hat{C} \otimes C)$ with certain properties. To express these, we use a manageable multiplicative unitary $\mathbb{W} \in \mathcal{U}(\mathcal{H} \otimes \mathcal{H})$ that generates $C$; in particular, $\mathbb{W}$ satisfies the pentagon equation

$$
\mathbb{W}_{23} \mathbb{W}_{12}=\mathbb{W}_{12} \mathbb{W}_{13} \mathbb{W}_{23} \quad \text { in } \mathcal{U}(\mathcal{H} \otimes \mathcal{H} \otimes \mathcal{H})
$$

Then $C$ and $\hat{C}$ act faithfully on $\mathcal{H}$. Write $\mathbb{P}$ for $\mathrm{P}$ viewed as an operator on $\mathcal{H} \otimes \mathcal{H}$. The condition that $\mathrm{P}$ is a bicharacter is equivalent to

$$
\mathbb{P}_{23} \mathbb{W}_{12}=\mathbb{W}_{12} \mathbb{P}_{13} \mathbb{P}_{23} \quad \text { and } \quad \mathbb{W}_{23} \mathbb{P}_{12}=\mathbb{P}_{12} \mathbb{P}_{13} \mathbb{W}_{23} \quad \text { in } \mathcal{U}(\mathcal{H} \otimes \mathcal{H} \otimes \mathcal{H})
$$

The condition that $\mathrm{P}$ is idempotent for the composition of quantum group homomorphisms is equivalent to the pentagon equation for $\mathbb{P}$ :

$$
\mathbb{P}_{23} \mathbb{P}_{12}=\mathbb{P}_{12} \mathbb{P}_{13} \mathbb{P}_{23} \quad \text { in } \mathcal{U}(\mathcal{H} \otimes \mathcal{H} \otimes \mathcal{H}) .
$$

Thus a $\mathrm{C}^{*}$-quantum group with projection is determined by two unitaries $\mathbb{W}, \mathbb{P} \in$ $\mathcal{U}(\mathcal{H} \otimes \mathcal{H})$ that satisfy (1.1)-(1.3); in addition, $\mathbb{W}$ must be manageable. Equation (1.3) means that $\mathbb{P}$ is a multiplicative unitary in its own right. It is manageable if $\mathbb{W}$ is. The $\mathrm{C}^{*}$-quantum group $\left(A, \Delta_{A}\right)$ it generates is the image of the projection.

It is much more difficult to describe the other factor $B$. As a $\mathrm{C}^{*}$-algebra, it should be the generalised fixed-point algebra for a canonical coaction of $\left(A, \Delta_{A}\right)$ on $\left(C, \Delta_{C}\right)$. In the group case, this says that $\mathrm{C}_{0}(H)$ is the generalised fixed-point algebra for the left or right translation action of $G$ on $\mathrm{C}_{0}(G \ltimes H)$. Unless $G$ is compact, this requires Rieffel's generalisation of fixed-point algebras to group actions that are "proper" in a suitable sense (see [9, 16]). Buss [2,3] has generalised this theory to locally compact quantum groups. We only need the special case of quantum homogeneous spaces, which is also treated by Vaes [24. All these approaches need some regularity assumptions on $\left(A, \Delta_{A}\right)$ and are technically difficult.

We may avoid these difficulties by staying on the level of multiplicative unitaries. We already described a $\mathrm{C}^{*}$-quantum group with projection through two multiplicative unitaries $\mathbb{W}, \mathbb{P} \in \mathcal{U}(\mathcal{H} \otimes \mathcal{H})$ on the same Hilbert space that are linked by the conditions (1.2). We find that any such pair comes from a "braided multiplicative unitary" over the $\mathrm{C}^{*}$-quantum group $\left(A, \Delta_{A}\right)$ generated by $\mathbb{P}$. 
A braided multiplicative unitary is a unitary $\mathbb{F} \in \mathcal{U}(\mathcal{L} \otimes \mathcal{L})$ for a Hilbert space $\mathcal{L}$ with a Yetter-Drinfeld module structure over $\left(A, \Delta_{A}\right)$. That is, $\mathcal{L}$ carries corepresentations $\mathrm{U} \in \mathcal{U}(\mathbb{K}(\mathcal{L}) \otimes A)$ and $\mathrm{V} \in \mathcal{U}(\mathbb{K}(\mathcal{L}) \otimes \hat{A})$ that are linked by a YetterDrinfeld commutation relation. In addition, $\mathbb{F}$ is equivariant for the tensor product corepresentations $\mathrm{U} \oplus \mathrm{U}$ and $\mathrm{V} \oplus \mathrm{V}$ on $\mathcal{L} \otimes \mathcal{L}$ and satisfies the braided pentagon equation:

$$
\mathbb{F}_{23} \mathbb{F}_{12}=\mathbb{F}_{12}\left(\mathcal{L}_{\chi^{\mathcal{L}}}\right)_{23} \mathbb{F}_{12}\left(\mathcal{L}_{\chi^{\mathcal{L}}}\right)_{23}^{*} \mathbb{F}_{23} \quad \text { in } \mathcal{U}(\mathcal{L} \otimes \mathcal{L} \otimes \mathcal{L})
$$

Here $\mathcal{L}_{\chi^{\mathcal{L}}}$ denotes the braiding operator on the tensor product of the Yetter-Drinfeld Hilbert space $\mathcal{L}$ with itself, see [12].

Since $A$ and $\hat{A}$ are represented faithfully on $\mathcal{H}$, the unitaries $\mathrm{U}$ and $\mathrm{V}$ are determined by their images $\mathbb{U}$ and $\mathbb{V}$ in $\mathcal{U}(\mathcal{L} \otimes \mathcal{H})$. It is convenient to replace $\mathbb{V}$ by $\hat{\mathbb{V}}:=\Sigma \mathbb{V}^{*} \Sigma \in \mathcal{U}(\mathcal{H} \otimes \mathcal{L})$. We also write $\mathbb{W}$ instead of $\mathbb{P}$; the multiplicative unitary for the semidirect product quantum group will be denoted by $\mathbb{W}^{C}$.

Thus a braided multiplicative unitary is a family of four unitaries $\mathbb{W} \in \mathcal{U}(\mathcal{H} \otimes \mathcal{H})$, $\mathbb{U} \in \mathcal{U}(\mathcal{L} \otimes \mathcal{H}), \hat{\mathbb{V}} \in \mathcal{U}(\mathcal{H} \otimes \mathcal{L})$, and $\mathbb{F} \in \mathcal{U}(\mathcal{L} \otimes \mathcal{L})$ for two Hilbert spaces $\mathcal{H}$ and $\mathcal{L}$; these unitaries satisfy seven conditions: the pentagon condition for $\mathbb{W}$; one corepresentation condition each for $\mathbb{U}$ and $\hat{\mathbb{V}}$, which link them to $\mathbb{W}$; the YetterDrinfeld condition linking $\mathbb{U}$ and $\hat{\mathbb{V}}$; the equivariance of $\mathbb{F}$ with respect to $\mathbb{U} \oplus \mathbb{U}$ and $\hat{\mathbb{V}} \oplus \hat{\mathbb{V}}$; and the braided pentagon equation for $\mathbb{F}$. We show that given these four unitaries subject to these seven conditions, the unitary

$$
\mathbb{W}_{1234}^{C}:=\mathbb{W}_{13} \mathbb{U}_{23} \hat{\mathbb{V}}_{34}^{*} \mathbb{F}_{24} \hat{\mathbb{V}}_{34} \quad \text { in } \mathcal{U}(\mathcal{H} \otimes \mathcal{L} \otimes \mathcal{H} \otimes \mathcal{L})
$$

is multiplicative. Furthermore, the unitaries $\mathbb{W}^{C}$ and $\mathbb{P}:=\mathbb{W}_{13} \mathbb{U}_{23}$ on $\mathcal{H} \otimes \mathcal{L} \otimes$ $\mathcal{H} \otimes \mathcal{L}$ satisfy the conditions (1.1)-(1.3) that characterise $\mathrm{C}^{*}$-quantum groups with projection. The only analytic issue is to prove that $\mathbb{W}^{C}$ is manageable if the braided multiplicative unitary is manageable in a suitable sense. Otherwise, the claim is proved by a direct computation. This has to be lengthy, however, because all seven conditions on our four unitaries must play their role.

Conversely, let $\mathbb{W}^{C}, \mathbb{P} \in \mathcal{U}(\mathcal{H} \otimes \mathcal{H})$ be unitaries satisfying the conditions (1.1)(1.3), with $\mathbb{W}^{C}$ manageable. Then we construct a braided multiplicative unitary based on the unitary $\mathbb{W}=\mathbb{P} \in \mathcal{U}(\mathcal{H} \otimes \mathcal{H})$, that is, we construct a Hilbert space $\mathcal{L}$ and unitaries $\mathbb{U} \in \mathcal{U}(\mathcal{L} \otimes \mathcal{H}), \hat{\mathbb{V}} \in \mathcal{U}(\mathcal{H} \otimes \mathcal{L})$, and $\mathbb{F} \in \mathcal{U}(\mathcal{L} \otimes \mathcal{L})$ satisfying the conditions for a braided multiplicative unitary, and we check that this braided multiplicative unitary is manageable. When we construct a pair $\left(\mathbb{W}^{C}, \mathbb{P}\right)$ out of this data as in (1.5), then we do not get back the same data we started with because the underlying Hilbert spaces have changed. We show, however, that the resulting $\mathrm{C}^{*}$-quantum groups with projection are the same. This isomorphism is also implemented by a quantum group isomorphism in the category constructed in [10].

When we start with a manageable braided multiplicative unitary, form the crossed product as in (1.5) and go back, we also get a different braided multiplicative unitary, which should be "equivalent" to the one we started with. Since we do not discuss how a braided multiplicative unitary generates a braided $\mathrm{C}^{*}$-quantum bialgebra, we cannot yet express this equivalence.

We treat one example of a braided multiplicative unitary in detail, namely, the one that defines the simplified quantum $\mathrm{E}(2)$ group, a variant of the quantum $\mathrm{E}(2)$ group introduced by Woronowicz in [26. We write down the braided multiplicative unitary and check that it is manageable. Similar computations appear in [1,26]. 


\section{Projections on Quantum Groups}

A $\mathrm{C}^{*}$-quantum group is, by definition, a $\mathrm{C}^{*}$-bialgebra that is generated by a manageable multiplicative unitary, see 21,28. We do not assume a $\mathrm{C}^{*}$-quantum group to have Haar weights. We fix a $\mathrm{C}^{*}$-quantum group $\mathbb{H}=\left(C, \Delta_{C}\right)$ and let $\mathbb{W} \in \mathcal{U}(\mathcal{H} \otimes \mathcal{H})$ be a manageable multiplicative unitary on a Hilbert space $\mathcal{H}$ that generates it. Let $\hat{\mathbb{H}}=\left(\hat{C}, \hat{\Delta}_{C}\right)$ be the dual quantum group.

A bialgebra morphism $\left(A, \Delta_{A}\right) \rightarrow\left(C, \Delta_{C}\right)$ between two $\mathrm{C}^{*}$-bialgebras is a $\mathrm{C}^{*}$-algebra morphism $f: A \rightarrow C$ (that is, a nondegenerate *-homomorphism $A \rightarrow \mathcal{M}(C)$ ) making the following diagram commute:

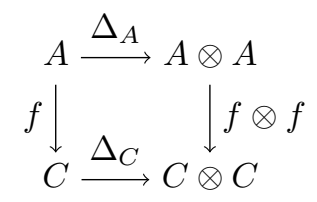

This notion of morphism is too restrictive, however, because a group homomorphism $G \rightarrow H$ need not induce a morphism $\mathrm{C}_{\mathrm{r}}^{*}(G) \rightarrow \mathrm{C}_{\mathrm{r}}^{*}(H)$. When we speak of morphisms of $\mathrm{C}^{*}$-quantum groups, we will mean those introduced by $\mathrm{Ng}$ [14], and we shall use the equivalent characterisations of these morphisms in [10].

Definition 2.1. A $\mathrm{C}^{*}$-quantum group with projection is a $\mathrm{C}^{*}$-quantum group with an idempotent quantum group endomorphism.

Before we make this definition explicit, we consider the commutative case. It allows us to view $\mathrm{C}^{*}$-quantum groups with projection as $\mathrm{C}^{*}$-quantum group analogues of semidirect products of groups.

Proposition 2.2. Let $\left(C, \Delta_{C}\right)$ be a commutative $\mathrm{C}^{*}$-quantum group with projection. Then $C \cong \mathrm{C}_{0}(G \ltimes H)$ for a semidirect product group, with the corresponding comultiplication, and the projection on $C$ comes from the group homomorphism $G \ltimes H \rightarrow G \ltimes H,(g, h) \mapsto\left(g, 1_{H}\right)$; here $G$ and $H$ are locally compact groups and $G$ acts continuously on $H$ by automorphisms. Conversely, any semidirect product group gives a commutative $\mathrm{C}^{*}$-quantum group with projection in this way.

Proof. Since $C$ is commutative, $C \cong \mathrm{C}_{0}(K)$ for a locally compact group $K$. A quantum group homomorphism from $C$ to itself is equivalent to a group homomorphism $K \rightarrow K$, and the composition of quantum group homomorphisms also corresponds to the composition of group homomorphisms. Thus a projection on $C$ corresponds to a group homomorphism $p: K \rightarrow K$ with $p \circ p=p$. Let $G \subseteq K$ and $H \subseteq K$ be the image and kernel of $p$, respectively; these are locally compact groups as well. Since $H$ is a normal subgroup, conjugation in $K$ lets $G \subseteq K$ act continuously on $H$ by automorphisms. The continuous maps $m: G \times H \rightarrow K,(g, h) \mapsto g \cdot h$, and $n: K \rightarrow G \times H, k \mapsto\left(p(k), p\left(k^{-1}\right) k\right)$, are inverse to each other and hence homeomorphisms. The multiplication is given by

$$
m\left(g_{1}, h_{1}\right) \cdot m\left(g_{2}, h_{2}\right)=g_{1} h_{1} g_{2} h_{2}=g_{1} g_{2}\left(g_{2}^{-1} h_{1} g_{2}\right) h_{2}=m\left(g_{1} g_{2},\left(g_{2}^{-1} h_{1} g_{2}\right) h_{2}\right) .
$$

Thus the homeomorphism $m$ is also a group isomorphism $K \cong G \ltimes H$. The converse assertion is routine to check.

Now we make Definition 2.1] explicit in several different ways, corresponding to some of the equivalent characterisations of quantum group morphisms in [10]. First we use unitaries satisfying pentagon equations. 
Proposition 2.3. $A \mathrm{C}^{*}$-quantum group with projection is given by a Hilbert space $\mathcal{H}$ and two unitaries $\mathbb{P}, \mathbb{W} \in \mathcal{U}(\mathcal{H} \otimes \mathcal{H})$ that satisfy

$$
\begin{aligned}
\mathbb{W}_{23} \mathbb{W}_{12} & =\mathbb{W}_{12} \mathbb{W}_{13} \mathbb{W}_{23}, \\
\mathbb{P}_{23} \mathbb{W}_{12} & =\mathbb{W}_{12} \mathbb{P}_{13} \mathbb{P}_{23}, \\
\mathbb{W}_{23} \mathbb{P}_{12} & =\mathbb{P}_{12} \mathbb{P}_{13} \mathbb{W}_{23}, \\
\mathbb{P}_{23} \mathbb{P}_{12} & =\mathbb{P}_{12} \mathbb{P}_{13} \mathbb{P}_{23} \quad \text { in } \mathcal{U}(\mathcal{H} \otimes \mathcal{H} \otimes \mathcal{H}) .
\end{aligned}
$$

In addition, $\mathbb{W}$ is manageable as a multiplicative unitary.

All four equations in Proposition 2.3 are variants of the pentagon equation.

Proof. [10, Lemma 3.2] describes a quantum group morphism from $\mathbb{H}$ to itself by a unitary $\mathbb{P} \in \mathcal{U}(\mathcal{H} \otimes \mathcal{H})$ on the same Hilbert space $\mathcal{H}$ on which the manageable multiplicative unitary $\mathbb{W}$ lives, subject to the two conditions (1.2), which are the second and third equation in our statement. The first equation is the pentagon equation for $\mathbb{W}$. The fourth equation says that the quantum group endomorphism associated to $\mathbb{P}$ is idempotent by [10, Definition 3.5].

Our first goal is to prove the following structural result:

Proposition 2.4. Any idempotent endomorphism $p: \mathbb{H} \rightarrow \mathbb{H}$ of a $\mathrm{C}^{*}$-quantum group $\mathbb{H}$ splits. That is, there are a $\mathrm{C}^{*}$-quantum group $\mathbb{G}$ and quantum group morphisms $a: \mathbb{G} \rightarrow \mathbb{H}, b: \mathbb{H} \rightarrow \mathbb{G}$ with $a \circ b=p$ and $b \circ a=\mathrm{id}_{\mathbb{G}}$.

The $\mathrm{C}^{*}$-quantum group $\mathbb{G}$ is called the image of the idempotent endomorphism $p$. We first construct this image, then we describe $a$ and $b$ and then we prove $a \circ b=p$ and $b \circ a=\operatorname{id}_{\mathbb{G}}$. The proof of Proposition 2.4 will be finished by Lemma 2.7.

The fourth equation in Proposition 2.3 says that $\mathbb{P}$ is a multiplicative unitary.

Proposition 2.5. The multiplicative unitary $\mathbb{P} \in \mathcal{U}(\mathcal{H} \otimes \mathcal{H})$ is manageable.

Proof. The multiplicative unitary $\mathbb{W}$ is manageable by assumption. This requires the existence of certain auxiliary operators $Q$ and $\widetilde{\mathbb{W}}$. We use the same operator $Q$ for $\mathbb{P}$. [28, Theorem 1.6] gives a unitary $\widetilde{\mathbb{P}} \in \mathcal{U}(\overline{\mathcal{H}} \otimes \mathcal{H})$ with

$$
(x \otimes u|\mathbb{P}| z \otimes y)=\left(\bar{z} \otimes Q u|\widetilde{\mathbb{P}}| \bar{x} \otimes Q^{-1} y\right)
$$

for all $x, z \in \mathcal{H}, u \in \mathcal{D}(Q)$ and $y \in \mathcal{D}\left(Q^{-1}\right)$. Lemma A.2 shows that $\mathbb{P}$ commutes with $Q \otimes Q$. So $\widetilde{\mathbb{P}}$ and $Q$ witness the manageability of the multiplicative unitary $\mathbb{P}$ (see [28, Definition 1.2]).

Proposition 2.5] shows that $\mathbb{P}$ generates a $\mathrm{C}^{*}$-quantum group $\mathbb{G}=\left(A, \Delta_{A}\right)$, which is called the image of $\mathbb{P}$. Let $\hat{\mathbb{G}}=\left(\hat{A}, \hat{\Delta}_{A}\right)$ be its dual.

The unitary $\mathbb{P}$ is the image of a unitary multiplier $\mathrm{P} \in \mathcal{U}(\hat{C} \otimes C)$ by [10, Lemma 3.2]. Hence slices of $\mathbb{P}$ are multipliers of $\hat{C} \subseteq \mathbb{B}(\mathcal{H})$ and $C \subseteq \mathbb{B}(\mathcal{H})$, respectively. These slices generate $\hat{A}$ and $A$, respectively, so $A \subseteq \mathcal{M}(C)$ and $\hat{A} \subseteq \mathcal{M}(\hat{C})$.

Lemma 2.6. The embeddings $i: A \rightarrow \mathcal{M}(C)$ and $j: \hat{A} \rightarrow \mathcal{M}(\hat{C})$ are bialgebra morphisms $\mathbb{G} \rightarrow \mathbb{H}$ and $\hat{\mathbb{G}} \rightarrow \hat{\mathbb{H}}$.

Proof. First we claim that $i$ and $j$ are $\mathrm{C}^{*}$-algebra morphisms, that is, $i(A) \cdot C=C$ and $j(\hat{A}) \cdot \hat{C}=\hat{C}$. The third condition in Proposition 2.3 is equivalent to

$$
\mathbb{P}_{12}^{*} \mathbb{W}_{23} \mathbb{P}_{12}=\mathbb{P}_{13} \mathbb{W}_{23} \quad \text { in } \mathcal{U}(\mathcal{H} \otimes \mathcal{H} \otimes \mathcal{H}) .
$$

When we slice the first two legs on both sides by $\omega_{1} \otimes \omega_{2}$ for $\omega_{1}, \omega_{2} \in \mathbb{B}(\mathcal{H})_{*}$ and close in norm, we get $C=A \cdot C$. The same argument works for $j$. 
The conditions in Proposition 2.3 also imply

$$
\begin{aligned}
& \mathbb{W}_{23} \mathbb{P}_{12} \mathbb{W}_{23}^{*}=\mathbb{P}_{12} \mathbb{P}_{13}=\mathbb{P}_{23} \mathbb{P}_{12} \mathbb{P}_{23}^{*}, \\
& \mathbb{W}_{12}^{*} \mathbb{P}_{23} \mathbb{W}_{12}=\mathbb{P}_{13} \mathbb{P}_{23}=\mathbb{P}_{12}^{*} \mathbb{P}_{23} \mathbb{P}_{12}
\end{aligned}
$$

Since $\left(\operatorname{id}_{D} \otimes \Delta_{C}\right)(x)=\mathbb{W}_{23} x_{12} \mathbb{W}_{23}^{*}$ for all $x \in D \otimes C$ and $\left(\operatorname{id}_{D} \otimes \Delta_{A}\right)(x)=\mathbb{P}_{23} x_{12} \mathbb{P}_{23}^{*}$ for all $x \in D \otimes A$, the first equation says that $\operatorname{id}_{\hat{A}} \otimes \Delta_{C}$ and $\operatorname{id}_{\hat{A}} \otimes \Delta_{A}$ agree on $\mathbb{P}$. Since slices of $\mathbb{P}$ generate $A$, this implies $\left.\Delta_{C}\right|_{A}=\Delta_{A}$, that is, $i$ is a bialgebra morphism. So is $j$ by a similar argument.

The bialgebra morphisms $i$ and $j$ give quantum group morphisms

$$
\begin{array}{ll}
V_{i}=(\mathrm{id} \otimes i)\left(\mathrm{W}^{A}\right) \in \mathcal{U}(\hat{A} \otimes C) & \text { from } A \text { to } C, \\
\hat{V}_{j}=(j \otimes \mathrm{id})\left(\mathrm{W}^{A}\right) \in \mathcal{U}(\hat{C} \otimes A) & \text { from } C \text { to } A .
\end{array}
$$

The quantum groups $\mathbb{G}$ and $\mathbb{H}$ may be generated by the multiplicative unitaries $\mathbb{P}$ and $\mathbb{W}$ on the same Hilbert space $\mathcal{H}$. Then the unitaries $V_{i}$ and $\hat{V}_{j}$ are both represented by the same unitary $\mathbb{P}$ on $\mathcal{H} \otimes \mathcal{H}$; the conditions in Proposition 2.3 allow us to view $\mathbb{P}$ as a quantum group homomorphism $\mathbb{G} \rightarrow \mathbb{H}, \mathbb{H} \rightarrow \mathbb{G}, \mathbb{H} \rightarrow \mathbb{H}$, or as the identity quantum group homomorphism on $\mathbb{G}$.

Lemma 2.7. The composite quantum group homomorphism $V_{i} \circ \hat{V}_{j}: \mathbb{H} \rightarrow \mathbb{G} \rightarrow \mathbb{H}$ is the given projection $\mathrm{P} \in \mathcal{U}(\hat{C} \otimes C)$ on $\mathbb{H}$. The other composite $\mathbb{G} \rightarrow \mathbb{H} \rightarrow \mathbb{G}$ is the identity on $\mathbb{G}$.

Proof. The composition of quantum group homomorphisms is described in [10] by a pentagon-like equation. The two claims in the lemma are both equivalent to the pentagon equation for $\mathbb{P}$.

The description of a projection on a $\mathrm{C}^{*}$-quantum group by a pair of bialgebra morphisms $(i, j)$ is unwieldy because it mixes quantum groups and their duals and because the composition $\mathbb{G} \rightarrow \mathbb{H} \rightarrow \mathbb{G}$ is computed only indirectly.

The quantum group morphism $\mathbb{H} \rightarrow \mathbb{G}$ is usually not representable by a bialgebra morphism $C \rightarrow A$. We may, however, also represent the quantum group morphism $j$ by a bialgebra morphism $\hat{j}^{\mathrm{u}}: C^{\mathrm{u}} \rightarrow A^{\mathrm{u}}$ between the universal quantum groups, see [10, Theorem 4.8]. Similarly, $i$ lifts to a bialgebra morphism $i^{\mathrm{u}}: A^{\mathrm{u}} \rightarrow C^{\mathrm{u}}$. A $\mathrm{C}^{*}$-quantum group with projection is equivalent to a $\mathrm{C}^{*}$-quantum group $\mathbb{H}$ with a bialgebra morphism $p: C^{\mathrm{u}} \rightarrow C^{\mathrm{u}}$ satisfying $p \circ p=p$ by [10, Theorem 4.8]. Our analysis above shows that for any such $p$ there are a $\mathrm{C}^{*}$-quantum group $\left(A, \Delta_{A}\right)$ and bialgebra morphisms $\hat{j}^{\mathrm{u}}: C^{\mathrm{u}} \rightarrow A^{\mathrm{u}}$ and $i^{\mathrm{u}}: A^{\mathrm{u}} \rightarrow C^{\mathrm{u}}$ with $p=i^{\mathrm{u}} \circ \hat{j}^{\mathrm{u}}$ and $\hat{j}^{\mathrm{u}} \circ i^{\mathrm{u}}=$ $\operatorname{id}_{A}$. Thus a quantum group with projection is equivalent to two $\mathrm{C}^{*}$-quantum groups with bialgebra morphisms $\hat{j}^{\mathrm{u}}: C^{\mathrm{u}} \rightarrow A^{\mathrm{u}}$ and $i^{\mathrm{u}}: A^{\mathrm{u}} \rightarrow C^{\mathrm{u}}$ with $\hat{j}^{\mathrm{u}} \circ i^{\mathrm{u}}=\operatorname{id}_{A}$.

Next we replace $\hat{j}$ by right and left quantum group morphisms:

Proposition 2.8. $A \mathrm{C}^{*}$-quantum group with projection is equivalent to two $\mathrm{C}^{*}$-quantum groups $\mathbb{H}=\left(C, \Delta_{C}\right)$ and $\mathbb{G}=\left(A, \Delta_{A}\right)$ with morphisms $i: A \rightarrow C$ and $\Delta_{R}: C \rightarrow C \otimes A$ such that the following diagrams commute:
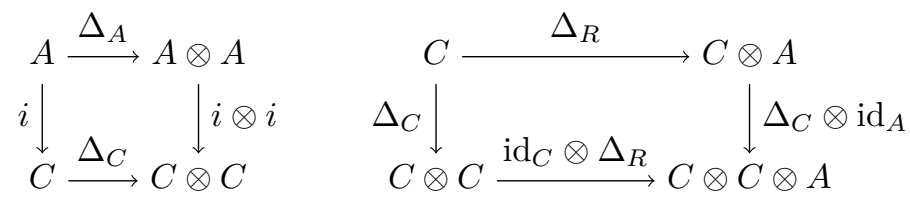


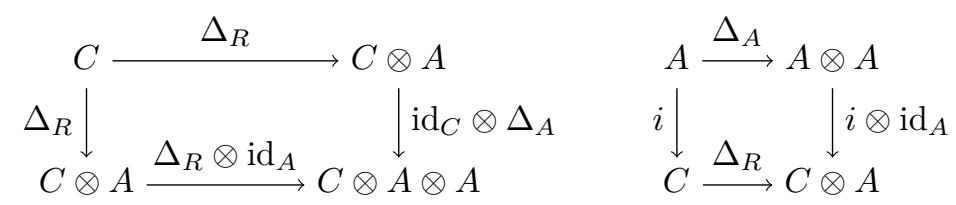

Another equivalent set of data is a pair of morphisms $i: A \rightarrow C$ and $\Delta_{L}: C \rightarrow A \otimes C$ with commutative diagrams

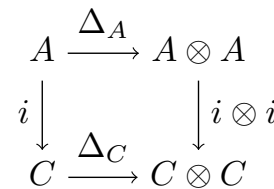

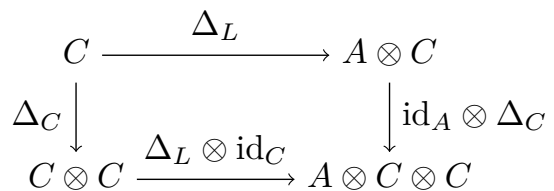

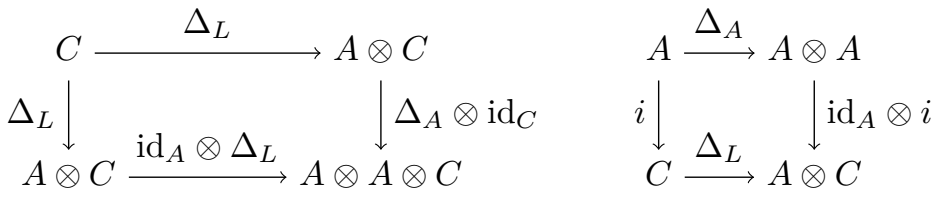

Finally, the quantum group with projection is equivalent to a triple of morphisms $i: A \rightarrow C, \Delta_{R}: C \rightarrow C \otimes A$ and $\Delta_{L}: C \rightarrow A \otimes C$ satisfying all the above conditions and, in addition,

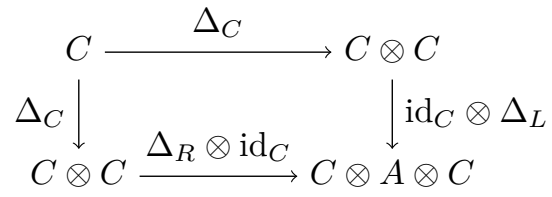

Then the following diagram also commutes:

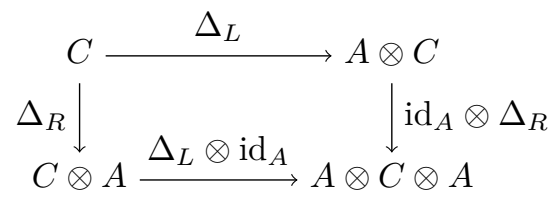

Proof. We have already seen that any projection on a $\mathrm{C}^{*}$-quantum group $\mathbb{H}$ has an image $\mathbb{G}$ and that there are a bialgebra morphism $i: A \rightarrow C$ and a quantum group morphism $\hat{j}: \mathbb{H} \rightarrow \mathbb{G}$ with $\hat{j} \circ i=\mathrm{id}_{\mathbb{G}}$ and $i \circ \hat{j}=p$, where $p$ denotes the given projection on $\mathbb{H}$. Now we describe $\hat{j}$ by a right quantum group morphism $\Delta_{R}$ as in 10, Definition 5.1].

The first diagram above says that $i$ is a bialgebra morphism. The second and third diagram together say that $\Delta_{R}$ is a right quantum group homomorphism from $C$ to $A$. The fourth diagram says that the composite $A \rightarrow C \rightarrow A$ of these quantum group morphisms is the identity map. Therefore, the other composite $C \rightarrow A \rightarrow C$ is idempotent, hence a projection. Thus $i$ and $\Delta_{R}$ give a projection on $\mathbb{H}$ with image $\mathbb{G}$. Conversely, any projection on a $\mathrm{C}^{*}$-quantum group $\mathbb{H}$ has an image by Proposition 2.4, which gives $i$ and $\Delta_{R}$ as above.

Replacing right by left quantum group morphisms shows that pairs $\left(i, \Delta_{L}\right)$ as above are also equivalent to $\mathrm{C}^{*}$-quantum groups with projection. Of the two diagrams that relate $\Delta_{R}$ and $\Delta_{L}$, the first one characterises when the right and left quantum group homomorphisms $\Delta_{R}$ and $\Delta_{L}$ describe the same quantum group morphism, and the second one commutes automatically, see [10, Lemma 5.7]. 
Let $A$ and $B$ be $\mathrm{C}^{*}$-algebras and $T \in \mathcal{U}(A \otimes B)$. Then $B$ is generated by $T$ in the sense of [27, Definition 4.1] if, for any representation $\xi: B \rightarrow \mathbb{B}(\mathcal{H})$ and any $\mathrm{C}^{*}$-algebra $C \subset \mathbb{B}(\mathcal{H})$, the condition $\left(\operatorname{id}_{A} \otimes \xi\right) T \in \mathcal{M}(A \otimes C)$ implies that $\xi \in \operatorname{Mor}(B, C)$.

Definition 2.9 ([4, Definition 3.2]). Let $\mathbb{I}=\left(C, \Delta_{C}\right)$ and $\mathbb{G}=\left(A, \Delta_{A}\right)$ be quantum groups. We call $\mathbb{G}$ a closed quantum subgroup of $\mathbb{I}$ in the sense of Woronowicz if there is a bicharacter $\mathrm{V} \in \mathcal{U}(\hat{C} \otimes A)$ that generates $\mathbb{G}$.

In the situation of Proposition 2.8, $\left(A, \Delta_{A}\right)$ is indeed a closed quantum subgroup of $\left(C, \Delta_{C}\right)$ because the bicharacter $\left(j \otimes \mathrm{id}_{A}\right)\left(\mathrm{W}^{A}\right) \in \mathcal{U}(\hat{C} \otimes A)$ generates $A$. This is to be expected because $\left(A, \Delta_{A}\right)$ is even a retract of $\left(C, \Delta_{C}\right)$ in the category of quantum group morphisms.

2.1. Semidirect products. In this section, we are going to show that the semidirect product construction in [12, Section 6] gives examples of $\mathrm{C}^{*}$-quantum groups with projection. Since we do not use this construction in the rest of the article, we do not recall the notation and setup from [12]. Readers unfamiliar with the semidirect product construction in 12 may skip this section.

Let $\mathbb{G}=\left(A, \Delta_{A}\right)$ be a $C^{*}$-quantum group. Let $(B, \beta, \hat{\beta})$ be an $A$-Yetter-Drinfeld algebra, that is, $\beta: B \rightarrow B \otimes A$ and $\hat{\beta}: B \rightarrow B \otimes \hat{A}$ are continuous coactions of $A$ and $\hat{A}$ that satisfy the compatibility condition in [12, Definition 5.11]. The twisted tensor product $B \otimes B=B \bigotimes_{\mathrm{W}} B$ is defined in [12]. We also require a coassociative comultiplication $\Delta_{B}: B \rightarrow B \otimes B$. Then [12, Theorem 6.8] describes a coassociative comultiplication $\Delta_{C}$ on $C:=A \otimes B$ and shows that the $\mathrm{C}^{*}$-bialgebra $\mathbb{H}=\left(C, \Delta_{C}\right)$ is bisimplifiable if $\left(B, \Delta_{B}\right)$ is bisimplifiable. Furthermore, $\Delta_{C}$ is injective if and only if $\Delta_{B}$ is injective. It is not studied in [12] when $\left(C, \Delta_{C}\right)$ is a $\mathrm{C}^{*}$-quantum group: by our definition, this would require a multiplicative unitary that generates it. If $C$ is unital, then this automatically exists and we are dealing with a compact quantum group. In the non-compact case, we need some sort of multiplicative unitary for $B$ to get one for $C$.

For now, we disregard this issue. We want to describe a projection on $\mathbb{H}$ with image $\mathbb{G}$, and the description of projections in Proposition 2.8 makes sense in our situation. Thus we are going to define morphisms

$$
i: A \rightarrow C, \quad \Delta_{R}: C \rightarrow C \otimes A, \quad \Delta_{L}: C \rightarrow A \otimes C
$$

with the properties listed in Proposition 2.8. If we know for some reason that $\mathbb{H}$ is a $\mathrm{C}^{*}$-quantum group, that is, comes from a manageable multiplicative unitary, then $\left(i, \Delta_{L}, \Delta_{R}\right)$ as in Proposition 2.8 give a projection on $\mathbb{H}$ with image $\mathbb{G}$. Actually, we only need either $\Delta_{L}$ or $\Delta_{R}$ for this purpose. We provide both, however, and check all conditions in Proposition 2.8.

The morphism $i: A \rightarrow A \otimes B=C$ is the canonical embedding from the twisted tensor product, which is denoted $j_{1}$ or $\iota_{A}$ in [12]. The right coaction $\Delta_{R}: C \rightarrow C \otimes A$ is the one constructed in [12, Lemma 6.5]. It is the unique one for which the embeddings $i=\iota_{A}: A \rightarrow C$ and $\iota_{B}: B \rightarrow C$ are equivariant; that is,

$$
\Delta_{R}\left(\iota_{A}(a) \cdot \iota_{B}(b)\right)=\left(\iota_{A} \otimes \operatorname{id}_{A}\right)\left(\Delta_{A}(a)\right) \cdot\left(\iota_{B} \otimes \operatorname{id}_{A}\right)(\beta(b)) .
$$

To construct $\Delta_{L}$, we equip $A \otimes A$ with the right $A$-coaction $\operatorname{id}_{A} \otimes \Delta_{A}$ on the second tensor factor; this is a continuous $A$-coaction, and $\Delta_{A}: A \rightarrow A \otimes A$ is an $A$-equivariant morphism. Therefore, there is an $A$-equivariant morphism $\Delta_{A} \otimes$ $\operatorname{id}_{B}: A \otimes B \rightarrow(A \otimes A) \otimes B$. We let $\Delta_{L}$ be the composite of $\Delta_{A} \otimes$ id $_{B}$ with the isomorphism $(A \otimes A) \otimes B \cong A \otimes(A \otimes B)=A \otimes C$ from [12, Lemma 3.14]. We may also rewrite

$$
A \bigotimes_{\mathrm{W}} B \cong B \bigotimes_{\widehat{\mathrm{W}}} A
$$


by [12, Proposition 5.1]. This is exactly the reduced crossed product for the $\hat{A}$-coaction on $B$ by [12, Section 6.3]. After this identification, $\Delta_{L}$ becomes the dual coaction on the reduced crossed product as described in [12, Section 6.3].

Proposition 2.10. The morphisms $i, \Delta_{R}$ and $\Delta_{L}$ constructed above make all the diagrams in Proposition 2.8 commute.

Proof. Of the ten diagrams in Proposition 2.8, the last one commutes automatically if the others do, and the first and fifth one are the same. So we have to check eight commuting diagrams. The maps $\Delta_{C}, \Delta_{R}$ and $\Delta_{L}$ are defined to have certain composites with $\iota_{A}$ and $\iota_{B}$ :

$$
\begin{array}{ll}
\Delta_{C} \circ \iota_{A}=\left(\iota_{A} \otimes \iota_{A}\right) \Delta_{A}, & \Delta_{C} \circ \iota_{B}=\Psi_{23} \circ \Delta_{B}, \\
\Delta_{R} \circ \iota_{A}=\left(\iota_{A} \otimes \mathrm{id}_{A}\right) \Delta_{A}, & \Delta_{R} \circ \iota_{B}=\left(\iota_{B} \otimes \mathrm{id}_{A}\right) \beta, \\
\Delta_{L} \circ \iota_{A}=\left(\operatorname{id}_{A} \otimes \iota_{A}\right) \Delta_{A}, & \Delta_{L} \circ \iota_{B}=1_{A} \otimes \iota_{B},
\end{array}
$$

where $\Psi_{23}: B \otimes B \rightarrow C \otimes C$ is the restriction of the map $\Psi$ in [12, Proposition 6.6] to the second two legs; that is, $\Psi_{23} j_{1}(b)=\left(\iota_{B} \otimes \iota_{A}\right) \beta(b)$ and $\Psi_{23} j_{2}(b)=\left(1 \otimes \iota_{B}\right)(b)$ for all $b \in B$.

In particular, $\left(\iota_{A} \otimes \iota_{A}\right) \circ \Delta_{A}=\Delta_{C} \circ \iota_{A}$ says that the first and fifth diagram commute, $\Delta_{R} \circ \iota_{A}=\left(\iota_{A} \otimes \mathrm{id}_{A}\right) \Delta_{A}$ says that the fourth diagram commutes, and $\Delta_{L} \circ \iota_{A}=\left(\mathrm{id}_{A} \otimes \iota_{A}\right) \Delta_{A}$ says that the eighth diagram commutes.

The remaining diagrams in Proposition 2.8 involve equalities of two maps defined on $C$. Two maps $f, f^{\prime}$ defined on $C$ are equal if and only if $f \circ \iota_{A}=f^{\prime} \circ \iota_{A}$ and $f \circ \iota_{B}=f^{\prime} \circ \iota_{B}$. For all remaining diagrams, it is trivial to check that they commute after composing with $\iota_{A}$ because of the explicit formulas above. The third and seventh diagram do not involve $\Delta_{C}$, so the composites with $\iota_{B}$ are also given explicitly, which makes them trivial to check; in fact, they say simply that $\Delta_{R}$ and $\Delta_{L}$ are a right and a left coaction, respectively, which is already checked in $[12]$.

The condition on $B$ for the sixth diagram is also trivial because $\Delta_{L}$ only does something complicated on $\iota_{A}(A)$ and $\Delta_{C}$ maps $\iota_{B}(B)$ into $\iota_{B}(B) \otimes C$.

For the second diagram, we must check $\left(\Delta_{C} \otimes \operatorname{id}_{A}\right) \Delta_{R} \iota_{B}=\left(\operatorname{id}_{C} \otimes \Delta_{R}\right) \Delta_{C} \iota_{B}$. Since $\Delta_{B}$ is $A$-equivariant, $\left(\Delta_{B} \otimes \operatorname{id}_{A}\right) \circ \beta=(\beta \bowtie \beta) \circ \Delta_{B}$. Using the definition of $\Delta_{C}$, we may rewrite our goal as $\left(\Psi_{23} \otimes \operatorname{id}_{A}\right)(\beta \bowtie \beta) \Delta_{B}=\left(\operatorname{id}_{C} \otimes \Delta_{R}\right) \Psi_{23} \Delta_{B}$. From this, we may cancel the factor $\Delta_{B}$, so it suffices to check that

$$
\left(\Psi_{23} \otimes \operatorname{id}_{A}\right)(\beta \bowtie \beta)=\left(\operatorname{id}_{C} \otimes \Delta_{R}\right) \Psi_{23}
$$

This is an equality of maps $B \otimes B \rightarrow C \otimes C \otimes A$, which we may check on both legs separately. On the first leg, this reduces to the condition $\left(\operatorname{id}_{B} \otimes \Delta_{A}\right) \beta=\left(\beta \otimes \mathrm{id}_{A}\right) \beta$ that says that $\beta$ is a coaction, and on the second leg this is trivial. This finishes the proof that the second diagram commutes

In the condition from the ninth diagram on $B$, we may cancel the factor $\Delta_{B}$ from $\Delta_{C}$, so it suffices to check that $\left(\operatorname{id}_{C} \otimes \Delta_{L}\right) \Psi_{23}=\left(\Delta_{R} \otimes \operatorname{id}_{C}\right) \Psi_{23}$ as maps $B \otimes B \rightarrow C \otimes A \otimes C$. This is once again checked separately on the two factors $B$. So we must check that the maps $\operatorname{id}_{C} \otimes \Delta_{L}$ and $\Delta_{R} \otimes \operatorname{id}_{C}$ take the same values both on $\left(\iota_{B} \otimes \iota_{A}\right) \beta(b)$ and on $1 \otimes \iota_{B}(b)$ for all $b \in B$. This reduces to the coaction condition for $\beta$ on $\left(\iota_{B} \otimes \iota_{A}\right) \beta(b)$ and is trivial on $1 \otimes \iota_{B}(b)$.

\section{Braided Multiplicative Unitaries}

The definition of a braided multiplicative unitary is as complicated as the definition of a braided $\mathrm{C}^{*}$-quantum group. Recall that the latter is relative to a $\mathrm{C}^{*}$-quantum group $\mathbb{G}=\left(A, \Delta_{A}\right)$ which generates the braiding. The underlying $\mathrm{C}^{*}$-algebra $B$ of a braided $\mathrm{C}^{*}$-quantum group carries continuous coactions $\beta$ and $\hat{\beta}$ 
of $\mathbb{G}$ and $\hat{\mathbb{G}}$, respectively, which satisfy the Yetter-Drinfeld compatibility condition which characterises coactions of the quantum codouble of $\mathbb{G}$. Finally, there is the comultiplication $\Delta_{B}: B \rightarrow B \otimes B$, which is equivariant with respect to $\beta$ and $\hat{\beta}$ and coassociative. Thus a braided $\mathrm{C}^{*}$-quantum group contains four coactions or comultiplications $\Delta_{A}, \beta, \hat{\beta}, \Delta_{B}$, which must satisfy seven algebraic conditions:

(1) $\Delta_{A}$ is coassociative;

(2) $\beta$ is a coaction of $\left(A, \Delta_{A}\right)$;

(3) $\hat{\beta}$ is a coaction of $\left(\hat{A}, \hat{\Delta}_{A}\right)$;

(4) $\beta$ and $\hat{\beta}$ satisfy the Drinfeld commutation relation, so that they give a coaction of the quantum codouble;

(5) $\Delta_{B}$ is equivariant with respect to the coaction $\beta$;

(6) $\Delta_{B}$ is equivariant with respect to the coaction $\hat{\beta}$;

(7) $\Delta_{B}$ is coassociative.

The tensor product $\otimes$ is not symmetric unless $\mathbb{G}$ is trivial. Thus $X \nabla^{\prime} Y:=Y \otimes X$ gives another equally reasonable tensor product. We may also consider braided quantum groups where the comultiplication takes values in $B \nabla^{\prime} B$ instead of $B \otimes B$. Actually, these $C^{*}$-algebras are canonically isomorphic through the flip map, which interchanges the two factors $B$. Thus there are two kinds of braided $\mathrm{C}^{*}$-quantum group, and taking the "coopposite," that is, composing $\Delta_{B}$ with the flip map $\Sigma$ and leaving everything else the same, gives a bijection between the two types.

Remark 3.1. The definition above simplifies somewhat if $\mathbb{G}$ is quasitriangular. Then a corepresentation $\beta$ determines a corepresentation $\hat{\beta}$ so as to form a coaction of the quantum codouble. Since $\hat{\beta}$ is a coaction constructed naturally from $\beta$, the conditions (3), (4) and (6) above are redundant. A similar simplification occurs for braided multiplicative unitaries. Since we are concerned with the general theory here, we do not explore this situation any further.

When we turn to multiplicative unitaries, we replace $\mathrm{C}^{*}$-algebras by Hilbert spaces on which they act faithfully; comultiplications and coactions are replaced by unitaries on appropriate tensor product Hilbert spaces that implement the coactions through conjugation. So to specify a braided multiplicative unitary, we need two Hilbert spaces and four unitaries that satisfy seven conditions, which correspond to the seven conditions for the comultiplications and coactions listed above. Moreover, there are two slightly different kinds of braided multiplicative unitaries, depending on whether we use the "standard" braiding or its opposite; which braiding is standard and which is opposite is, of course, a mere convention. The following definition contains the details:

Definition 3.2. Let $\mathcal{H}$ and $\mathcal{L}$ be Hilbert spaces and let $\mathbb{W} \in \mathcal{U}(\mathcal{H} \otimes \mathcal{H})$ be a manageable multiplicative unitary; in particular, $\mathbb{W}$ satisfies the pentagon equation

$$
\mathbb{W}_{23} \mathbb{W}_{12}=\mathbb{W}_{12} \mathbb{W}_{13} \mathbb{W}_{23}
$$

A top-braided multiplicative unitary on $\mathcal{L}$ relative to $\mathbb{W}$ is given by unitaries

$$
\mathbb{U} \in \mathcal{U}(\mathcal{L} \otimes \mathcal{H}), \quad \hat{\mathbb{V}} \in \mathcal{U}(\mathcal{H} \otimes \mathcal{L}), \quad \mathbb{F} \in \mathcal{U}(\mathcal{L} \otimes \mathcal{L})
$$

which satisfy the following conditions:

- $\mathbb{U}$ is a right corepresentation of $\mathbb{W}$ :

$$
\mathbb{W}_{23} \mathbb{U}_{12}=\mathbb{U}_{12} \mathbb{U}_{13} \mathbb{W}_{23} \quad \text { in } \mathcal{U}(\mathcal{L} \otimes \mathcal{H} \otimes \mathcal{H})
$$

- $\hat{\mathbb{V}}$ is a left corepresentation of $\mathbb{W}$ :

$$
\hat{\mathbb{V}}_{23} \mathbb{W}_{12}=\mathbb{W}_{12} \hat{\mathbb{V}}_{13} \hat{\mathbb{V}}_{23} \quad \text { in } \mathcal{U}(\mathcal{H} \otimes \mathcal{H} \otimes \mathcal{L}) ;
$$


- the corepresentations $\mathbb{U}$ and $\hat{\mathbb{V}}$ are Drinfeld compatible:

$$
\mathbb{U}_{23} \mathbb{W}_{13} \hat{\mathbb{V}}_{12}=\hat{\mathbb{V}}_{12} \mathbb{W}_{13} \mathbb{U}_{23} \quad \text { in } \mathcal{U}(\mathcal{H} \otimes \mathcal{L} \otimes \mathcal{H})
$$

- $\mathbb{F}$ is invariant with respect to the right corepresentation $\mathbb{U} \oplus \mathbb{U}:=\mathbb{U}_{13} \mathbb{U}_{23}$ of $\mathbb{W}$ on $\mathcal{L} \otimes \mathcal{L}$ :

$$
\mathbb{U}_{13} \mathbb{U}_{23} \mathbb{F}_{12}=\mathbb{F}_{12} \mathbb{U}_{13} \mathbb{U}_{23} \quad \text { in } \mathcal{U}(\mathcal{L} \otimes \mathcal{L} \otimes \mathcal{H})
$$

- $\mathbb{F}$ is invariant with respect to the left corepresentation $\hat{\mathbb{V}} \oplus \hat{\mathbb{V}}:=\hat{\mathbb{V}}_{13} \hat{\mathbb{V}}_{12}$ of $\mathbb{W}$ on $\mathcal{L} \otimes \mathcal{L}$ :

$$
\hat{\mathbb{V}}_{13} \hat{\mathbb{V}}_{12} \mathbb{F}_{23}=\mathbb{F}_{23} \hat{\mathbb{V}}_{13} \hat{\mathbb{V}}_{12} \quad \text { in } \mathcal{U}(\mathcal{H} \otimes \mathcal{L} \otimes \mathcal{L}) ;
$$

- $\mathbb{F}$ satisfies the top-braided pentagon equation

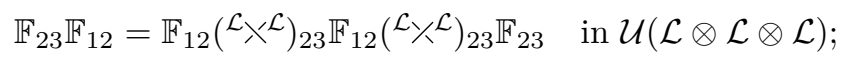

here the braiding $\mathcal{L}_{\chi^{\mathcal{L}}} \in \mathcal{U}(\mathcal{L} \otimes \mathcal{L})$ and $\mathcal{L}_{\chi^{\mathcal{L}}}=\left(\mathcal{L}_{\chi^{\mathcal{L}}}\right)^{*}$ are defined as $\mathcal{L}_{\chi^{\mathcal{L}}}=$ $Z \Sigma$ for the flip $\Sigma, x \otimes y \mapsto y \otimes x$, and the unique unitary $Z \in \mathcal{U}(\mathcal{L} \otimes \mathcal{L})$ that satisfies

$$
Z_{13}=\hat{\mathbb{V}}_{23} \mathbb{U}_{12}^{*} \hat{\mathbb{V}}_{23}^{*} \mathbb{U}_{12} \quad \text { in } \mathcal{U}(\mathcal{L} \otimes \mathcal{H} \otimes \mathcal{L}) .
$$

A bottom-braided multiplicative unitary on $\mathcal{L}$ relative to $\mathbb{W}$ is given by the same unitaries $\mathbb{U}, \hat{\mathbb{V}}, \mathbb{F}$ satisfying (3.4)-(3.8) and the bottom-braided pentagon equation

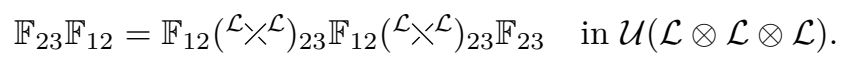

Two corepresentations $\mathbb{U}$ and $\hat{\mathbb{V}}$ on a Hilbert space $\mathcal{L}$ satisfying (3.6) are equivalent to a corepresentation of the quantum codouble of the quantum group associated to $\mathbb{W}$. It is shown in [12 that these corepresentations form a braided monoidal category. Our conventions differ from those in 12 because we use a left corepresentation $\hat{\mathbb{V}}$ instead of the corresponding right corepresentation $\mathbb{V}:=\Sigma \hat{\mathbb{V}}^{*} \Sigma$. The compatibility condition (3.6) and the definition of the braiding operator above are equivalent to those in [12] up to this change of notation. The operator $Z$ in (3.10) exists because $\mathbb{W}$ is manageable. It is shown in [12] that the operators ${ }^{\mathcal{L}_{1}} \gamma^{\mathcal{L}_{2}}$ defined as above form a braiding on the tensor category of triples $(\mathcal{L}, \mathbb{U}, \hat{\mathbb{V}})$; the operators $\mathcal{L}_{1}$ 人 $^{\mathcal{L}_{2}}$ give the opposite braiding.

In a braided monoidal category, the leg numbering notation should use the braiding operators. This explains why we replace $\mathbb{F}_{13}$ by $\left(\mathcal{L}_{\chi^{\mathcal{L}}}\right)_{23} \mathbb{F}_{12}\left(\mathcal{L}_{\chi^{\mathcal{L}}}\right)_{23}$ or

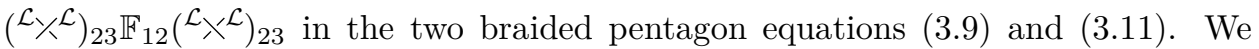
should also have replaced $\mathbb{F}_{23}$ by $\mathcal{L}_{\chi^{\mathcal{L}} \otimes \mathcal{L}} \mathbb{F}_{12} \mathcal{L}{ } \mathcal{L} \otimes \mathcal{L}$; the braiding operator $\mathcal{L}_{\chi^{\mathcal{L}} \otimes \mathcal{L}}$ is defined as $Z^{\prime} \Sigma^{\mathcal{L}, \mathcal{L} \otimes \mathcal{L}}$, where $Z^{\prime}$ is the unique operator on $(\mathcal{L} \otimes \mathcal{L}) \otimes \mathcal{L}$ with

$$
Z_{134}^{\prime}=(\hat{\mathbb{V}} \oplus \hat{\mathbb{V}})_{234} \mathbb{U}_{12}^{*}(\hat{\mathbb{V}} \oplus \hat{\mathbb{V}})_{234}^{*} \mathbb{U}_{12} \quad \text { in } \mathcal{U}(\mathcal{L} \otimes \mathcal{H} \otimes \mathcal{L} \otimes \mathcal{L}) .
$$

Since we are dealing with a braided monoidal category, we also have

$$
\mathcal{L}_{\chi^{\mathcal{L}} \otimes \mathcal{L}}=\mathcal{L}_{\chi^{\mathcal{L}}} \mathcal{L}_{23} \mathcal{L}_{\chi^{\mathcal{L}}}{ }_{12}, \quad \mathcal{L} \otimes \mathcal{L}_{\chi^{\mathcal{L}}}=\mathcal{L}_{\chi^{\mathcal{L}}}{ }_{12} \mathcal{L}_{\chi^{\mathcal{L}}} \mathcal{L}_{23} .
$$

Since $\mathbb{F}$ is invariant with respect to both corepresentations, it commutes with any operator that is constructed in a natural way out of them, such as $Z^{\prime}$. This implies

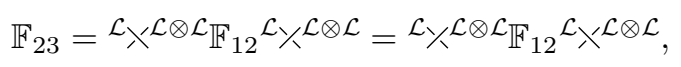

so here the braiding has no effect. This also implies

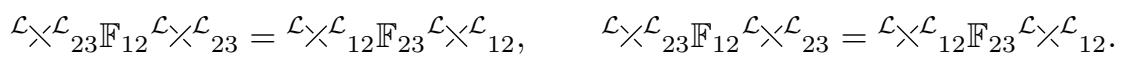


Such equations are easier to digest as pictures:

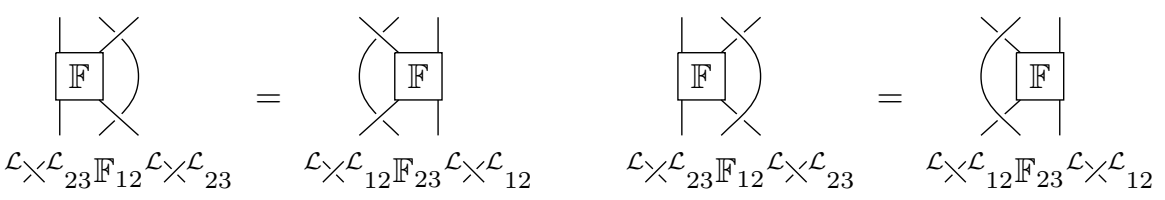

The top-braided pentagon equation (3.9) uses the version of $\mathbb{F}_{13}$ where $\mathbb{F}$ acts on the two top strands, whereas the bottom-braided pentagon equation (3.11) uses the version of $\mathbb{F}_{13}$ where $\mathbb{F}$ acts on the two bottom strands; this explains our notation.

The braided pentagon equation is the usual pentagon equation if and only if $\mathbb{F}$ commutes with $\Sigma Z \Sigma$. Sufficient conditions for this are $Z=1, \mathbb{U}=1$ or $\hat{\mathbb{V}}=1$.

From now on, we restrict attention to top-braided multiplicative unitaries, so braided multiplicative unitary means top-braided multiplicative unitary.

Definition 3.12. The dual of a braided multiplicative unitary $(\mathbb{U}, \hat{\mathbb{V}}, \mathbb{F})$ over $\mathbb{W}$ is $(\mathbb{V}, \hat{\mathbb{U}}, \widehat{\mathbb{F}})$ over $\widehat{\mathbb{W}}$, where $\widehat{\mathbb{W}}:=\Sigma \mathbb{W}^{*} \Sigma, \mathbb{V}:=\Sigma \hat{\mathbb{V}}^{*} \Sigma, \hat{\mathbb{U}}:=\Sigma \mathbb{U}^{*} \Sigma$, and

$$
\widehat{\mathbb{F}}:=\mathcal{L}_{\mathcal{L}} \mathcal{L}_{\mathbb{F}^{*}} \mathcal{L}_{\nearrow} \mathcal{L} \in \mathcal{U}(\mathcal{L} \otimes \mathcal{L}) .
$$

The braiding operator $\mathcal{L}_{\chi^{\mathcal{L}}}$ for $(\widehat{\mathbb{W}}, \mathbb{V}, \hat{\mathbb{U}})$ is the opposite braiding $\mathcal{L}_{\aleph^{\mathcal{L}}}$ for $(\mathbb{W}, \mathbb{U}, \hat{\mathbb{V}})$. Therefore, the dual of the dual is the braided multiplicative unitary that we started with, even if the braiding is not symmetric.

Proposition 3.13. Let $(\mathbb{U}, \hat{\mathbb{V}}, \mathbb{F})$ be a top-braided multiplicative unitary over $\mathbb{W}$. Its dual $(\mathbb{V}, \widehat{\mathbb{U}}, \widehat{\mathbb{F}})$ is a top-braided multiplicative unitary over $\widehat{\mathbb{W}}:=\Sigma \mathbb{W}^{*} \Sigma$.

Proof. It is well-known that the dual $\widehat{\mathbb{W}}$ is again a multiplicative unitary, that $\mathbb{U}$ is a right corepresentation of $\mathbb{W}$ if and only if $\hat{\mathbb{U}}$ is a left corepresentation of $\widehat{\mathbb{W}}$, and that $\hat{\mathbb{V}}$ is a left corepresentation of $\mathbb{W}$ if and only if $\mathbb{V}$ is a right corepresentation of $\widehat{\mathbb{W}}$. Routine computations show that the Drinfeld compatibility condition and the invariance conditions are also preserved by the duality. The top-braided (or bottom-braided) pentagon equation for the dual is equivalent to the top-braided (or bottom-braided) pentagon equation for the original braided multiplicative unitary because the duality replaces the braiding by the opposite braiding.

Now we define when a braided multiplicative unitary $(\mathbb{W}, \mathbb{U}, \hat{\mathbb{V}}, \mathbb{F})$ is manageable. This requires $\mathbb{W}$ to be manageable, that is, there are a strictly positive operator $Q$ on $\mathcal{H}$ and a unitary $\widetilde{\mathbb{W}} \in \mathcal{U}(\overline{\mathcal{H}} \otimes \mathcal{H})$ with $\mathbb{W}^{*}(Q \otimes Q) \mathbb{W}=Q \otimes Q$ and

$$
(x \otimes u|\mathbb{W}| z \otimes y)=\left(\bar{z} \otimes Q u|\widetilde{\mathbb{W}}| \bar{x} \otimes Q^{-1} y\right)
$$

for all $x, z \in \mathcal{H}, u \in \mathcal{D}(Q)$ and $y \in \mathcal{D}\left(Q^{-1}\right.$ ) (see [28, Definition 1.2]). Here $\overline{\mathcal{H}}$ is the conjugate Hilbert space, and an operator is strictly positive if it is positive and self-adjoint with trivial kernel. The condition $\mathbb{W}^{*}(Q \otimes Q) \mathbb{W}=Q \otimes Q$ means that the unitary $\mathbb{W}$ commutes with the unbounded operator $Q \otimes Q$.

Definition 3.15. Let $\mathbb{W} \in \mathcal{U}(\mathcal{H} \otimes \mathcal{H})$ be a manageable multiplicative unitary and let $Z$ and $Q$ be as above. A braided multiplicative unitary $(\mathbb{U}, \hat{\mathbb{V}}, \mathbb{F})$ over $\mathbb{W}$ is manageable if there are a strictly positive operator $Q_{\mathcal{L}}$ on $\mathcal{L}$ and a unitary $\widetilde{\mathbb{F}} \widetilde{Z}^{*} \in$ $\mathcal{U}(\overline{\mathcal{L}} \otimes \mathcal{L})$ such that

$$
\begin{aligned}
\mathbb{U}\left(Q_{\mathcal{L}} \otimes Q\right) \mathbb{U}^{*} & =Q_{\mathcal{L}} \otimes Q, \\
\hat{\mathbb{V}}\left(Q \otimes Q_{\mathcal{L}}\right) \hat{\mathbb{V}}^{*} & =Q \otimes Q_{\mathcal{L}}, \\
\mathbb{F}\left(Q_{\mathcal{L}} \otimes Q_{\mathcal{L}}\right) \mathbb{F}^{*} & =Q_{\mathcal{L}} \otimes Q_{\mathcal{L}}, \\
\left(x \otimes u\left|Z^{*} \mathbb{F}\right| y \otimes v\right) & =\left(\bar{y} \otimes Q_{\mathcal{L}}(u)\left|\widetilde{\mathbb{F}} \widetilde{Z}^{*}\right| \bar{x} \otimes Q_{\mathcal{L}}^{-1}(v)\right)
\end{aligned}
$$


for all $x, y \in \mathcal{L}, u \in \mathcal{D}\left(Q_{\mathcal{L}}\right)$ and $v \in \mathcal{D}\left(Q_{\mathcal{L}}^{-1}\right)$.

We have written $\widetilde{\mathbb{F}} \widetilde{Z}^{*}$ and not $\widetilde{\mathbb{F}}$ in (3.19) to make the formula more symmetric and to clarify the manageability of the dual of a braided multiplicative unitary.

We now describe the operator $\widetilde{Z}$ that we want to use. The corepresentation $\mathbb{U}$ of $\mathbb{W}$ on $\mathcal{L}$ induces a contragradient corepresentation on $\overline{\mathcal{L}}$. This is of the form $\widetilde{\mathbb{U}}^{*}$, where $\widetilde{\mathbb{U}} \in \mathcal{U}(\overline{\mathcal{L}} \otimes \mathcal{H})$ satisfies a variant of (3.14), see [28, Theorem 1.6] and [21, Proposition 10]. Since $\widetilde{\mathbb{U}}^{*}$ is a right corepresentation of $\mathbb{W}$ on $\overline{\mathcal{L}}$, there is a unique unitary $\widetilde{Z} \in \mathcal{U}(\overline{\mathcal{L}} \otimes \mathcal{L})$ that satisfies

$$
\widetilde{Z}_{13}=\hat{\mathbb{V}}_{23} \widetilde{\mathbb{U}}_{12} \hat{\mathbb{V}}_{23}^{*} \widetilde{\mathbb{U}}_{12}^{*} \quad \text { in } \mathcal{U}(\overline{\mathcal{L}} \otimes \mathcal{H} \otimes \mathcal{L})
$$

We use this unitary in (3.19). Of course, it does not matter which unitary $\widetilde{Z}$ we use because we may absorb it in $\widetilde{\mathbb{F}}$.

Proposition 3.21. The dual of a manageable braided multiplicative unitary is again manageable.

Proof. Let $(\mathbb{U}, \hat{\mathbb{V}}, \mathbb{F})$ be a manageable top-braided multiplicative unitary over $\mathbb{W}$, let $Z$ and $\widetilde{Z}$ be as in (3.10) and (3.20). Let $\widetilde{\mathbb{W}}, Q$ witness the manageability of $\mathbb{W}$ and let $\widetilde{\mathbb{F}}$ and $Q_{\mathcal{L}}$ witness the manageability of $(\mathbb{U}, \hat{\mathbb{V}}, \mathbb{F})$.

On $\mathcal{L} \otimes \mathcal{H} \otimes \mathcal{L}$, both $\mathbb{U}_{12}$ and $\hat{\mathbb{V}}_{23}$ commute with $Q_{\mathcal{L}} \otimes Q \otimes Q_{\mathcal{L}}$ by (3.16) and (3.17). Hence so does $Z$ by (3.10). Thus

$$
Z\left(Q_{\mathcal{L}} \otimes Q_{\mathcal{L}}\right) Z^{*}=Q_{\mathcal{L}} \otimes Q_{\mathcal{L}}
$$

Together with (3.18), this implies that $Z^{*} \mathbb{F}$ commutes with $Q_{\mathcal{L}} \otimes Q_{\mathcal{L}}$. This together with (3.19) implies that $\widetilde{\mathbb{F}} \widetilde{Z}^{*}$ commutes with $Q_{\mathcal{L}}^{\top} \otimes Q_{\mathcal{L}}^{-1}$, compare the proof of Lemma A.2 or [28, Proposition 1.4.(1)].

The unitary $\widetilde{\mathbb{U}}$ commutes with $Q_{\mathcal{L}}^{\top} \otimes Q^{-1}$, compare Lemma A.2 or [28, Proposition 1.4.(1)]. This together with (3.17) and (3.20) implies

$$
\widetilde{Z}\left(Q_{\mathcal{L}}^{\top} \otimes Q_{\mathcal{L}}^{-1}\right) \widetilde{Z}^{*}=Q_{\mathcal{L}}^{\top} \otimes Q_{\mathcal{L}}^{-1}
$$

compare the proof of (3.22). Hence

$$
\widetilde{\mathbb{F}}\left(Q_{\mathcal{L}}^{\top} \otimes Q_{\mathcal{L}}^{-1}\right) \widetilde{\mathbb{F}}^{*}=Q_{\mathcal{L}}^{\top} \otimes Q_{\mathcal{L}}^{-1}
$$

because $\widetilde{\mathbb{F}} \widetilde{Z}^{*}$ commutes with $Q_{\mathcal{L}}^{\top} \otimes Q_{\mathcal{L}}^{-1}$ as well.

If $y \in \mathcal{D}\left(Q_{\mathcal{L}}\right), x \in \mathcal{D}\left(Q_{\mathcal{L}}^{-1}\right)$, and $u, v \in \mathcal{L}$, then

$$
\left(x \otimes u\left|Z^{*} \mathbb{F}\right| y \otimes v\right)=\left(\overline{Q_{\mathcal{L}}(y)} \otimes u\left|\widetilde{\mathbb{F}} \widetilde{Z}^{*}\right| \overline{Q_{\mathcal{L}}^{-1}(x)} \otimes v\right)
$$

this is proved like [28, Proposition 1.4 (2)]. We rewrite this using the unitaries $\widetilde{\hat{Z}}, \widetilde{\mathbb{F}} \in \mathcal{U}(\overline{\mathcal{L}} \otimes \mathcal{L})$ defined by

$$
\widetilde{\hat{Z}}:=\left(\Sigma \widetilde{Z}^{*} \Sigma\right)^{\top \otimes \top}, \quad \widetilde{\widehat{\mathbb{F}}}:=\left(\Sigma \widetilde{\mathbb{F}}^{*} \Sigma\right)^{\top \otimes \top} .
$$


By definition, $\hat{Z}^{*} \widehat{\mathbb{F}}=\Sigma \mathbb{F}^{*} Z \Sigma$ and $\widetilde{\widehat{\mathbb{F}}} \hat{Z}^{*}=\left(\Sigma \widetilde{Z} \widetilde{\mathbb{F}}^{*} \Sigma\right)^{\top \otimes T}$. Thus (3.25) gives

$$
\begin{aligned}
\left(x \otimes u\left|\hat{Z}^{*} \widehat{\mathbb{F}}\right| y \otimes v\right) & =\left(x \otimes u\left|\Sigma \mathbb{F}^{*} Z \Sigma\right| y \otimes v\right) \\
& =\overline{\left(y \otimes v\left|\Sigma Z^{*} \mathbb{F} \Sigma\right| x \otimes u\right)} \\
& =\overline{\left(v \otimes y\left|Z^{*} \mathbb{F}\right| u \otimes x\right)} \\
& =\overline{\left(\overline{Q_{\mathcal{L}}(u)} \otimes y\left|\widetilde{\mathbb{F}} \widetilde{Z}^{*}\right| \overline{Q_{\mathcal{L}}^{-1}(v)} \otimes x\right)} \\
& \left.=\overline{\left(Q_{\mathcal{L}}^{-1}(v)\right.} \otimes x\left|\widetilde{Z} \widetilde{\mathbb{F}}^{*}\right| \overline{Q_{\mathcal{L}}(u)} \otimes y\right) \\
& =\left(x \otimes \overline{Q_{\mathcal{L}}^{-1}(v)}\left|\Sigma \widetilde{Z} \widetilde{\mathbb{F}} \widetilde{\mathbb{F}}^{*}\right| y \otimes \overline{Q_{\mathcal{L}}(u)}\right) \\
& =\left(\bar{y} \otimes Q_{\mathcal{L}}(u)\left|\left(\Sigma \widetilde{Z} \widetilde{\mathbb{F}}^{*} \Sigma\right)^{\top} \otimes \mathrm{T}\right| \bar{x} \otimes Q_{\mathcal{L}}^{-1}(v)\right) \\
& =\left(\bar{y} \otimes Q_{\mathcal{L}}(u)\left|\widetilde{\mathbb{F}} \widetilde{\tilde{Z}}^{*}\right| \bar{x} \otimes Q_{\mathcal{L}}^{-1}(v)\right) .
\end{aligned}
$$

Since the unitary $Z$ for the dual braided multiplicative unitary becomes $\hat{Z}$, the operators $Q_{\mathcal{L}}$ and $\widetilde{\mathbb{F}}$ witness the manageability of $\widehat{\mathbb{F}}$.

3.1. Semidirect product multiplicative unitaries. In this section, we construct a semidirect product multiplicative unitary $\mathbb{W}^{C}$ and a projection $\mathbb{P}$ out of a braided multiplicative unitary $(\mathbb{U}, \hat{\mathbb{V}}, \mathbb{F})$ over a multiplicative unitary $\mathbb{W}$. We show that the semidirect product multiplicative unitary $\mathbb{W}^{C}$ is manageable if the braided multiplicative unitary $(\mathbb{U}, \hat{\mathbb{V}}, \mathbb{F})$ is manageable.

The formulas and proofs below are explicit but lengthy because all four unitaries $\mathbb{W}, \mathbb{U}, \hat{\mathbb{V}}, \mathbb{F}$ must enter in the definitions of $\mathbb{W}^{C}$ and $\mathbb{P}$ and all seven conditions on them must be used in the proofs.

Theorem 3.26. Let $(\mathbb{U}, \hat{\mathbb{V}}, \mathbb{F})$ be a braided multiplicative unitary over a multiplicative unitary $\mathbb{W}$. Define $\mathbb{W}_{1234}^{C}, \mathbb{P} \in \mathcal{U}(\mathcal{H} \otimes \mathcal{L} \otimes \mathcal{H} \otimes \mathcal{L})$ by

$$
\begin{aligned}
\mathbb{W}_{1234}^{C} & :=\mathbb{W}_{13} \mathbb{U}_{23} \hat{\mathbb{V}}_{34}^{*} \mathbb{F}_{24} \hat{\mathbb{V}}_{34}, \\
\mathbb{P}_{1234} & :=\mathbb{W}_{13} \mathbb{U}_{23} .
\end{aligned}
$$

Then $\mathbb{W}^{C}$ and $\mathbb{P}$ satisfy the four pentagon-like equations in Proposition 2.3. Thus they give a $\mathrm{C}^{*}$-quantum group with projection when $\mathbb{W}^{C}$ is manageable.

Proof. We first verify the pentagon equation (3.3) for $\mathbb{W}_{1234}^{C}$. Let

$$
X X X=\mathbb{W}_{3456}^{C} \mathbb{W}_{1234}^{C}\left(\mathbb{W}^{C}\right)_{3456}^{*} .
$$

We will rewrite this in several steps using the conditions in Definition 3.2 We use $\{\ldots\}$ to highlight which part of the formula we are modifying in the following step. Definition (3.27) gives

$$
X X X=\mathbb{W}_{35}\left\{\mathbb{U}_{45} \hat{\mathbb{V}}_{56}^{*} \mathbb{F}_{46} \hat{\mathbb{V}}_{56}\right\}\left\{\mathbb{W}_{13} \mathbb{U}_{23}\right\} \hat{\mathbb{V}}_{34}^{*} \mathbb{F}_{24} \hat{\mathbb{V}}_{34} \hat{\mathbb{V}}_{56}^{*} \mathbb{F}_{46}^{*} \hat{\mathbb{V}}_{56} \mathbb{U}_{45}^{*} \mathbb{W}_{35}^{*} .
$$

Since $\mathbb{U}_{45} \hat{\mathbb{V}}_{56}^{*} \mathbb{F}_{46} \hat{\mathbb{V}}_{56}$ and $\mathbb{W}_{13} \mathbb{U}_{23}$ commute,

$$
X X X=\left\{\mathbb{W}_{35} \mathbb{W}_{13}\right\} \mathbb{U}_{23} \mathbb{U}_{45} \hat{\mathbb{V}}_{56}^{*} \mathbb{F}_{46}\left\{\hat{\mathbb{V}}_{56}\right\} \hat{\mathbb{V}}_{34}^{*} \mathbb{F}_{24} \hat{\mathbb{V}}_{34}\left\{\hat{\mathbb{V}}_{56}^{*}\right\} \mathbb{F}_{46}^{*} \hat{\mathbb{V}}_{56} \mathbb{U}_{45}^{*} \mathbb{W}_{35}^{*} .
$$

Now we use the pentagon equation (3.3) for $\mathbb{W}$ and commute $\hat{\mathbb{V}}_{56}$ with $\hat{\mathbb{V}}_{34}^{*} \mathbb{F}_{24} \hat{\mathbb{V}}_{34}$ :

$$
X X X=\mathbb{W}_{13} \mathbb{W}_{15}\left\{\mathbb{W}_{35} \mathbb{U}_{23}\right\} \mathbb{U}_{45} \hat{\mathbb{V}}_{56}^{*}\left\{\mathbb{F}_{46} \hat{\mathbb{V}}_{34}^{*}\right\} \mathbb{F}_{24}\left\{\hat{\mathbb{V}}_{34} \mathbb{F}_{46}^{*}\right\} \hat{\mathbb{V}}_{56} \mathbb{U}_{45}^{*} \mathbb{W}_{35}^{*} .
$$

Equations (3.4) and (3.8) turn this into

$$
\mathbb{W}_{13} \mathbb{W}_{15} \mathbb{U}_{23} \mathbb{U}_{25} \mathbb{W}_{35} \mathbb{U}_{45}\left\{\hat{\mathbb{V}}_{56}^{*} \hat{\mathbb{V}}_{34}^{*}\right\} \hat{\mathbb{V}}_{36}^{*} \mathbb{F}_{46}\left\{\hat{\mathbb{V}}_{36} \mathbb{F}_{24} \hat{\mathbb{V}}_{36}^{*}\right\} \mathbb{F}_{46}^{*} \hat{\mathbb{V}}_{36}\left\{\hat{\mathbb{V}}_{34} \hat{\mathbb{V}}_{56}\right\} \mathbb{U}_{45}^{*} \mathbb{W}_{35}^{*}
$$

Commuting $\hat{\mathbb{V}}_{56}$ with $\hat{\mathbb{V}}_{34}$ and $\hat{\mathbb{V}}_{36}$ with $\mathbb{F}_{24}$ gives

$$
X X X=\mathbb{W}_{13} \mathbb{W}_{15} \mathbb{U}_{23} \mathbb{U}_{25}\left\{\mathbb{W}_{35} \mathbb{U}_{45} \hat{\mathbb{V}}_{34}^{*}\right\} \hat{\mathbb{V}}_{56}^{*} \hat{\mathbb{V}}_{36}^{*} \mathbb{F}_{46} \mathbb{F}_{24} \mathbb{F}_{46}^{*} \hat{\mathbb{V}}_{36} \hat{\mathbb{V}}_{56}\left\{\hat{\mathbb{V}}_{34} \mathbb{U}_{45}^{*} \mathbb{W}_{35}^{*}\right\} .
$$


Now (3.6) gives

$$
X X X=\mathbb{W}_{13} \mathbb{W}_{15} \mathbb{U}_{23} \mathbb{U}_{25} \hat{\mathbb{V}}_{34}^{*} \mathbb{U}_{45}\left\{\mathbb{W}_{35} \hat{\mathbb{V}}_{56}^{*} \hat{\mathbb{V}}_{36}^{*}\right\} \mathbb{F}_{46} \mathbb{F}_{24} \mathbb{F}_{46}^{*}\left\{\hat{\mathbb{V}}_{36} \hat{\mathbb{V}}_{56} \mathbb{W}_{35}^{*}\right\} \mathbb{U}_{45}^{*} \hat{\mathbb{V}}_{34}
$$

We transform this using (3.5):

$$
X X X=\mathbb{W}_{13} \mathbb{W}_{15} \mathbb{U}_{23} \mathbb{U}_{25} \hat{\mathbb{V}}_{34}^{*} \mathbb{U}_{45} \hat{\mathbb{V}}_{56}^{*}\left\{\mathbb{W}_{35}\right\} \mathbb{F}_{46} \mathbb{F}_{24} \mathbb{F}_{46}^{*}\left\{\mathbb{W}_{35}^{*}\right\} \hat{\mathbb{V}}_{56} \mathbb{U}_{45}^{*} \hat{\mathbb{V}}_{34}
$$

We commute $\mathbb{W}_{35}$ with $\mathbb{F}_{46} \mathbb{F}_{24} \mathbb{F}_{46}^{*}$ :

$$
X X X=\mathbb{W}_{13} \mathbb{W}_{15} \mathbb{U}_{23} \mathbb{U}_{25} \hat{\mathbb{V}}_{34}^{*} \mathbb{U}_{45} \hat{\mathbb{V}}_{56}^{*}\left\{\mathbb{F}_{46} \mathbb{F}_{24} \mathbb{F}_{46}^{*}\right\} \hat{\mathbb{V}}_{56} \mathbb{U}_{45}^{*} \hat{\mathbb{V}}_{34} .
$$

Now we use the braided pentagon equation (3.9) and the definition of the braiding through $Z$ :

$$
X X X=\mathbb{W}_{13} \mathbb{W}_{15} \mathbb{U}_{23}\left\{\mathbb{U}_{25} \hat{\mathbb{V}}_{34}^{*}\right\} \mathbb{U}_{45}\left\{\hat{\mathbb{V}}_{56}^{*} \mathbb{F}_{24}\right\} Z_{46} \mathbb{F}_{26} Z_{46}^{*} \hat{\mathbb{V}}_{56} \mathbb{U}_{45}^{*} \hat{\mathbb{V}}_{34}
$$

Now we commute $\mathbb{U}_{25}$ with $\hat{\mathbb{V}}_{34}^{*}, \hat{\mathbb{V}}_{56}^{*}$ with $\mathbb{F}_{24}$ :

$$
X X X=\mathbb{W}_{13} \mathbb{W}_{15} \mathbb{U}_{23} \hat{\mathbb{V}}_{34}^{*} \mathbb{U}_{25} \mathbb{U}_{45} \mathbb{F}_{24}\left\{\hat{\mathbb{V}}_{56}^{*} Z_{46}\right\} \mathbb{F}_{26}\left\{Z_{46}^{*} \hat{\mathbb{V}}_{56} \mathbb{U}_{45}^{*}\right\} \hat{\mathbb{V}}_{34} .
$$

Equation (3.10) implies $\mathbb{U}_{45} \hat{\mathbb{V}}_{56}^{*} Z_{46}=\hat{\mathbb{V}}_{56}^{*} \mathbb{U}_{45}$, so this becomes

$$
X X X=\mathbb{W}_{13} \mathbb{W}_{15} \mathbb{U}_{23} \hat{\mathbb{V}}_{34}^{*}\left\{\mathbb{U}_{25} \mathbb{U}_{45} \mathbb{F}_{24} \mathbb{U}_{45}^{*}\right\} \hat{\mathbb{V}}_{56}^{*}\left\{\mathbb{U}_{45} \mathbb{F}_{26} \mathbb{U}_{45}^{*}\right\} \hat{\mathbb{V}}_{56} \hat{\mathbb{V}}_{34} .
$$

Now we use (3.7) and commute $\mathbb{F}_{26}$ with $\mathbb{U}_{45}$ :

$$
X X X=\mathbb{W}_{13}\left\{\mathbb{W}_{15}\right\}\left\{\mathbb{U}_{23} \hat{\mathbb{V}}_{34}^{*} \mathbb{F}_{24}\right\}\left\{\mathbb{U}_{25} \hat{\mathbb{V}}_{56}^{*} \mathbb{F}_{26} \hat{\mathbb{V}}_{56}\right\}\left\{\hat{\mathbb{V}}_{34}\right\} .
$$

Finally, we commute $\mathbb{W}_{15}$ with $\mathbb{U}_{23} \hat{\mathbb{V}}_{34}^{*} \mathbb{F}_{24}$ and $\mathbb{W}_{15} \mathbb{U}_{25} \hat{\mathbb{V}}_{56}^{*} \mathbb{F}_{26} \hat{\mathbb{V}}_{56}$ with $\hat{\mathbb{V}}_{34}$ to get

$$
X X X=\left\{\mathbb{W}_{13} \mathbb{U}_{23} \hat{\mathbb{V}}_{34}^{*} \mathbb{F}_{24} \hat{\mathbb{V}}_{34}\right\}\left\{\mathbb{W}_{15} \mathbb{U}_{25} \hat{\mathbb{V}}_{56}^{*} \mathbb{F}_{26} \hat{\mathbb{V}}_{56}\right\}=\mathbb{W}_{1234}^{C} \mathbb{W}_{1256}^{C} .
$$

This is the desired pentagon equation for $\mathbb{W}_{1234}^{C}$.

Next we show that $\mathbb{P}$ satisfies the pentagon equation:

$$
\begin{aligned}
\mathbb{P}_{3456} \mathbb{P}_{1234} \mathbb{P}_{3456} & =\mathbb{W}_{35} \mathbb{U}_{45} \mathbb{W}_{13} \mathbb{U}_{23} \mathbb{U}_{45}^{*} \mathbb{W}_{35}^{*}=\mathbb{W}_{35} \mathbb{W}_{13} \mathbb{U}_{23} \mathbb{W}_{35}^{*} \\
& =\mathbb{W}_{13} \mathbb{W}_{15} \mathbb{W}_{35} \mathbb{U}_{23} \mathbb{W}_{35}^{*}=\mathbb{W}_{13} \mathbb{W}_{15} \mathbb{U}_{23} \mathbb{U}_{25}=\mathbb{W}_{13} \mathbb{U}_{23} \mathbb{W}_{15} \mathbb{U}_{25} \\
& =\mathbb{P}_{1234} \mathbb{P}_{1256} .
\end{aligned}
$$

The first and last equalities are the definition of $\mathbb{P}$; the second step commutes $\mathbb{U}_{45}$ with $\mathbb{W}_{13} \mathbb{U}_{23}$; the third step uses the pentagon equation (3.3) for $\mathbb{W}$; the fourth step uses (3.4); the fifth step commutes $\mathbb{W}_{15}$ with $\mathbb{U}_{23}$.

Next we prove $\mathbb{P}_{3456} \mathbb{W}_{1234}^{C}=\mathbb{W}_{1234}^{C} \mathbb{P}_{1256} \mathbb{P}_{3456}$ or, equivalently, $\mathbb{P}_{3456} \mathbb{W}_{1234}^{C} \mathbb{P}_{3456}^{*}=$ $\mathbb{W}_{1234}^{C} \mathbb{P}_{1256}$ :

$$
\begin{aligned}
\mathbb{P}_{3456} \mathbb{W}_{1234}^{C} \mathbb{P}_{3456}^{*} & =\mathbb{W}_{35} \mathbb{U}_{45} \mathbb{W}_{13} \mathbb{U}_{23} \hat{\mathbb{V}}_{34}^{*} \mathbb{F}_{24} \hat{\mathbb{V}}_{34} \mathbb{U}_{45}^{*} \mathbb{W}_{35}^{*} \\
& =\mathbb{W}_{35} \mathbb{W}_{13} \mathbb{U}_{23} \mathbb{U}_{45} \hat{\mathbb{V}}_{34}^{*} \mathbb{F}_{24} \hat{\mathbb{V}}_{34} \mathbb{U}_{45}^{*} \mathbb{W}_{35}^{*} \\
& =\mathbb{W}_{13} \mathbb{W}_{15} \mathbb{W}_{35} \mathbb{U}_{23} \mathbb{U}_{45} \hat{\mathbb{V}}_{34}^{*} \mathbb{F}_{24} \hat{\mathbb{V}}_{34} \mathbb{U}_{45}^{*} \mathbb{W}_{35}^{*} \\
& =\mathbb{W}_{13} \mathbb{W}_{15} \mathbb{U}_{23} \mathbb{U}_{25} \mathbb{W}_{35} \mathbb{U}_{45} \hat{\mathbb{V}}_{34}^{*} \mathbb{F}_{24} \hat{\mathbb{V}}_{34} \mathbb{U}_{45}^{*} \mathbb{W}_{35}^{*} \\
& =\mathbb{W}_{13} \mathbb{U}_{23} \mathbb{W}_{15} \mathbb{U}_{25} \hat{\mathbb{V}}_{34}^{*} \mathbb{U}_{45} \mathbb{W}_{35} \mathbb{F}_{24} \mathbb{W}_{35}^{*} \mathbb{U}_{45}^{*} \hat{\mathbb{V}}_{34} \\
& =\mathbb{W}_{13} \mathbb{U}_{23} \hat{\mathbb{V}}_{34}^{*} \mathbb{W}_{15} \mathbb{U}_{25} \mathbb{U}_{45} \mathbb{F}_{24} \mathbb{U}_{45}^{*} \hat{\mathbb{V}}_{34} \\
& =\mathbb{W}_{13} \mathbb{U}_{23} \hat{\mathbb{V}}_{34}^{*} \mathbb{W}_{15} \mathbb{F}_{24} \mathbb{U}_{25} \hat{\mathbb{V}}_{34} \\
& =\mathbb{W}_{13} \mathbb{U}_{23} \hat{\mathbb{V}}_{34}^{*} \mathbb{F}_{24} \hat{\mathbb{V}}_{34} \mathbb{W}_{15} \mathbb{U}_{25}=\mathbb{W}_{1234}^{C} \mathbb{P}_{1256} .
\end{aligned}
$$

The first and last equalities are the definitions of $\mathbb{W} C$ and $\mathbb{P}$; the second, sixth, and eighth steps commute unitaries in different legs; the third step uses the pentagon equation (3.3) for $\mathbb{W}$; the fourth step uses (3.4); the fifth step uses (3.6) and commutes unitaries in different legs; the seventh step uses (3.7). 
Finally, we prove $\mathbb{W}_{3456}^{C} \mathbb{P}_{1234}=\mathbb{P}_{1234} \mathbb{P}_{1256} \mathbb{W}_{3456}^{C}$ by computing $\mathbb{P}_{1234}^{*} \mathbb{W}_{3456}^{C} \mathbb{P}_{1234}$ :

$$
\begin{aligned}
\mathbb{P}_{1234}^{*} \mathbb{W}_{3456}^{C} \mathbb{P}_{1234} & =\mathbb{U}_{23}^{*} \mathbb{W}_{13}^{*} \mathbb{W}_{35} \mathbb{U}_{45} \hat{\mathbb{V}}_{56}^{*} \mathbb{F}_{46} \hat{\mathbb{V}}_{56} \mathbb{W}_{13} \mathbb{U}_{23} \\
& =\mathbb{U}_{23}^{*} \mathbb{W}_{13}^{*} \mathbb{W}_{35} \mathbb{W}_{13} \mathbb{U}_{45} \hat{\mathbb{V}}_{56}^{*} \mathbb{F}_{46} \hat{\mathbb{V}}_{56} \mathbb{U}_{23} \\
& =\mathbb{U}_{23}^{*} \mathbb{W}_{15} \mathbb{W}_{35} \mathbb{U}_{45} \hat{\mathbb{V}}_{56}^{*} \mathbb{F}_{46} \hat{\mathbb{V}}_{56} \mathbb{U}_{23} \\
& =\mathbb{W}_{15} \mathbb{U}_{23}^{*} \mathbb{W}_{35} \mathbb{U}_{23} \mathbb{U}_{45} \hat{\mathbb{V}}_{56}^{*} \mathbb{F}_{46} \hat{\mathbb{V}}_{56} \\
& =\mathbb{W}_{15} \mathbb{U}_{25} \mathbb{W}_{35} \mathbb{U}_{45} \hat{\mathbb{V}}_{56}^{*} \mathbb{F}_{46} \hat{\mathbb{V}}_{56}=\mathbb{P}_{1256} \mathbb{W}_{3456}^{C} .
\end{aligned}
$$

The first and last steps are the definitions of $\mathbb{W}^{C}$ and $\mathbb{P}$; the second and fourth steps commute unitaries in different legs; the third step uses the pentagon equation (3.3) for $\mathbb{W}$; the fifth step uses (3.4).

Theorem 3.29. Let $\mathbb{W}$ be a manageable multiplicative unitary and let $(\mathbb{U}, \hat{\mathbb{V}}, \mathbb{F})$ be a manageable braided multiplicative unitary over $\mathbb{W}$. Then the multiplicative unitaries $\mathbb{W}^{C}:=\mathbb{W}_{13} \mathbb{U}_{23} \hat{\mathbb{V}}_{34}^{*} \mathbb{F}_{24} \hat{\mathbb{V}}_{34}$ and $\mathbb{P}:=\mathbb{W}_{13} \mathbb{U}_{23}$ are manageable.

Proof. Let $\widetilde{\mathbb{W}}$ and $Q$ witness the manageability of $\mathbb{W}$, and let $\widetilde{\mathbb{F}}$ and $Q_{\mathcal{L}}$ witness the manageability of $\mathbb{F}$. The construction of the unitary $\widetilde{\mathbb{U}}$ in (A.1) works for any right corepresentation of $\mathbb{W}$ by the same argument; in particular, it works for $\mathbb{U}$, so we get $\widetilde{\mathbb{U}} \in \mathcal{U}(\overline{\mathcal{L}} \otimes \mathcal{H})$ with

$$
\langle x \otimes u|\mathbb{U}| z \otimes y\rangle=\left\langle\bar{z} \otimes Q u|\widetilde{U}| \bar{x} \otimes Q^{-1} y\right\rangle
$$

for all $x, z \in \mathcal{L}, u \in \mathcal{D}(Q)$ and $y \in \mathcal{D}\left(Q^{-1}\right)$.

Let $Q^{C}:=Q \otimes Q_{\mathcal{L}} \in \mathcal{U}(\mathcal{H} \otimes \mathcal{L})$ and

$$
\widetilde{\mathbb{W}}_{1234}^{C}:=\widetilde{\mathbb{W}}_{13} \hat{\mathbb{V}}_{34}^{*} \widetilde{\mathbb{F}}_{24} \widetilde{\mathbb{U}}_{23} \hat{\mathbb{V}}_{34} \in \mathcal{U}(\overline{\mathcal{H}} \otimes \overline{\mathcal{L}} \otimes \mathcal{H} \otimes \mathcal{L}) .
$$

We claim that these operators witness the manageability of $\mathbb{W}^{C}$. It is clear that $Q^{C}$ is strictly positive.

The operators $\mathbb{W}_{13}, \hat{\mathbb{V}}_{34}, \mathbb{U}_{23}$ and $\mathbb{F}_{24}$ all commute with $Q^{C} \otimes Q^{C}=Q \otimes Q_{\mathcal{L}} \otimes$ $Q \otimes Q_{\mathcal{L}}$ by the manageability assumptions. Hence $\mathbb{W}^{C}$ commutes with $Q^{C} \otimes Q^{C}$. It remains to check (3.14) for $\mathbb{W}^{C}, \widetilde{\mathbb{W}^{C}}$ and $Q^{C}$. We relegate this technical computation to Lemma A.5 in the appendix. This finishes the proof that $\mathbb{W}^{C}$ is manageable. Now Proposition 2.5 shows that $\mathbb{P}$ is manageable as well.

3.2. Analysis of a quantum group with projection. In this section, we construct a braided multiplicative unitary from a quantum group with projection. Our starting point is a Hilbert space $\mathcal{H}$ with two unitaries $\mathbb{W}^{C}, \mathbb{P} \in \mathcal{U}(\mathcal{H} \otimes \mathcal{H})$ satisfying the conditions in Proposition 2.3. We must construct another Hilbert space $\mathcal{L}$ with operators $\mathbb{U} \in \mathcal{U}(\mathcal{L} \otimes \mathcal{H}), \hat{\mathbb{V}} \in \mathcal{U}(\mathcal{H} \otimes \mathcal{L})$ and $\mathbb{F} \in \mathcal{U}(\mathcal{L} \otimes \mathcal{L})$ as in Definition 3.2

In particular, the corepresentations $\mathbb{U}$ and $\hat{\mathbb{V}}$ form a Drinfeld pair for the multiplicative unitary $\mathbb{P}$. The simplest general construction of such a Drinfeld pair lives on the tensor product Hilbert space $\mathcal{L}:=\overline{\mathcal{H}} \otimes \mathcal{H}$, where $\overline{\mathcal{H}}$ denotes the conjugate Hilbert space of $\mathcal{H}$. Therefore, we will use this rather large Hilbert space.

Let $\mathbb{G}=\left(A, \Delta_{A}\right)$ be the $\mathrm{C}^{*}$-quantum group generated by $\mathbb{P}$, which is manageable by Proposition 2.5. Let $\mathbb{H}=\left(C, \Delta_{C}\right)$ be the $\mathrm{C}^{*}$-quantum group generated by the manageable multiplicative unitary $\mathbb{W}^{C}$. By construction, we have inclusion maps $\iota: C \rightarrow \mathbb{B}(\mathcal{H})$ and $\hat{\iota}: C \rightarrow \mathbb{B}(\mathcal{H})$, which are non-degenerate ${ }^{*}$-homomorphisms. The reduced bicharacter is the unique unitary $\mathrm{W}^{C} \in \mathcal{U}(\hat{C} \otimes C)$ with $\mathbb{W}^{C}=(\hat{\iota} \otimes \iota)\left(\mathrm{W}^{C}\right)$ or, briefly, $\mathbb{W}^{C}=\mathrm{W}_{\hat{\iota} \iota}^{C}$. By construction, $A \subseteq \mathcal{M}(C)$ and $\hat{A} \subseteq \mathcal{M}(\hat{C})$ as $\mathrm{C}^{*}$-subalgebras of $\mathbb{B}(\mathcal{H})$.

The representations $(\iota, \hat{\iota})$ form a Heisenberg pair for the quantum group $\left(C, \Delta_{C}\right)$ in the notation of [1]. This Heisenberg pair generates an anti-Heisenberg pair 
$\alpha: C \rightarrow \mathbb{B}(\overline{\mathcal{H}}), \hat{\alpha}: \hat{C} \rightarrow \mathbb{B}(\overline{\mathcal{H}})$ by [11, Lemma 3.6]. Thus

$$
\mathrm{W}_{1 \alpha}^{C} \mathrm{~W}_{\hat{\alpha} 3}^{C}=\mathrm{W}_{\hat{\alpha} 3}^{C} \mathrm{~W}_{13}^{C} \mathrm{~W}_{1 \alpha}^{C} \quad \text { in } \mathcal{U}(\hat{C} \otimes \mathbb{K}(\overline{\mathcal{H}}) \otimes \hat{C}) .
$$

The restriction of a Heisenberg or anti-Heisenberg pair for $\mathbb{H}$ to $\mathbb{G}$ remains a Heisenberg or anti-Heisenberg pair, respectively. Thus

$$
\mathrm{P}_{1 \alpha} \mathrm{P}_{\hat{\alpha} 3}=\mathrm{P}_{\hat{\alpha} 3} \mathrm{P}_{13} \mathrm{P}_{1 \alpha} \quad \text { in } \mathcal{U}(\hat{A} \otimes \mathbb{K}(\overline{\mathcal{H}}) \otimes \hat{A}) .
$$

To make computations shorter, we shall use leg numbering notation such as $\mathrm{P}_{i j}, \mathrm{~W}_{i j}^{C} \in$ $\mathcal{U}(\overline{\mathcal{H}} \otimes \mathcal{H} \otimes \overline{\mathcal{H}} \otimes \mathcal{H})$ for $1 \leq i<j \leq 4$. This means the unitary acting on the $i$ th and $j$ th tensor factor by applying the appropriate representations of $C$ or $\hat{C}$ to the two legs of $\mathrm{P}$ or $\mathrm{W}^{C}$, respectively. For instance, $\mathrm{P}_{12}=(\hat{\alpha} \otimes \iota)(\mathrm{P}) \otimes 1_{\overline{\mathcal{H}} \otimes \mathcal{H}}$. This notation is not ambiguous if we also specify the Hilbert space on which the operator acts because we have given one representation of $C$ and $\hat{C}$ on $\mathcal{H}$ and $\overline{\mathcal{H}}$ each. We let

$$
\begin{array}{ll}
\mathbb{U}:=\mathrm{P}_{23} \mathrm{P}_{13}:=(\hat{\iota} \otimes \iota) \mathrm{P}_{23} \cdot(\hat{\alpha} \otimes \iota) \mathrm{P}_{13} & \text { in } \mathcal{U}(\overline{\mathcal{H}} \otimes \mathcal{H} \otimes \mathcal{H}), \\
\hat{\mathbb{V}}:=\mathrm{P}_{12} \mathrm{P}_{13}:=(\hat{\iota} \otimes \alpha) \mathrm{P}_{12} \cdot(\hat{\iota} \otimes \iota) \mathrm{P}_{13} & \text { in } \mathcal{U}(\mathcal{H} \otimes \overline{\mathcal{H}} \otimes \mathcal{H}), \\
\mathbb{F}:=\mathrm{P}_{14}^{*} \mathrm{P}_{24}^{*} \mathrm{~W}_{24}^{C} \mathrm{~W}_{14}^{C} & \text { in } \mathcal{U}(\overline{\mathcal{H}} \otimes \mathcal{H} \otimes \overline{\mathcal{H}} \otimes \mathcal{H}) .
\end{array}
$$

Theorem 3.37. The unitaries $\mathbb{P} \in \mathcal{U}(\mathcal{H} \otimes \mathcal{H}), \mathbb{U} \in \mathcal{U}(\mathcal{L} \otimes \mathcal{H}), \hat{\mathbb{V}} \in \mathcal{U}(\mathcal{H} \otimes \mathcal{L})$, and $\mathbb{F} \in \mathcal{U}(\mathcal{L} \otimes \mathcal{L})$ form a braided multiplicative unitary.

The proof of Theorem 3.37 will take some work. The precise formulas for $\alpha$ and $\hat{\alpha}$ will only matter in the end when we check the manageability of our braided multiplicative unitary. Thus our construction really uses that $\mathbb{W}^{C}$ is manageable (or at least modular). The pentagon equation (3.3) for $\mathbb{P}$ holds by assumption. Equations (3.4) and (3.5), which say that $\mathbb{U}$ and $\hat{\mathbb{V}}$ are corepresentations, amount to

$$
\begin{array}{ll}
\mathrm{P}_{34} \mathrm{P}_{23} \mathrm{P}_{13}=\mathrm{P}_{23} \mathrm{P}_{13} \mathrm{P}_{24} \mathrm{P}_{14} \mathrm{P}_{34} & \text { in } \mathcal{U}(\overline{\mathcal{H}} \otimes \mathcal{H} \otimes \mathcal{H} \otimes \mathcal{H}), \\
\mathrm{P}_{23} \mathrm{P}_{24} \mathrm{P}_{12}=\mathrm{P}_{12} \mathrm{P}_{13} \mathrm{P}_{14} \mathrm{P}_{23} \mathrm{P}_{24} & \text { in } \mathcal{U}(\mathcal{H} \otimes \mathcal{H} \otimes \overline{\mathcal{H}} \otimes \mathcal{H}) .
\end{array}
$$

We get both equations using the pentagon equation for $\mathbb{P}$ twice, in legs where the representations of $\mathbb{H}$ and hence of $\mathbb{G}$ form a Heisenberg pair. Thus $\mathbb{U}$ and $\hat{V}$ are corepresentations of $\mathbb{P}$. The Drinfeld compatibility condition (3.6) becomes

$$
\mathrm{P}_{34} \mathrm{P}_{24} \mathrm{P}_{14} \mathrm{P}_{12} \mathrm{P}_{13}=\mathrm{P}_{12} \mathrm{P}_{13} \mathrm{P}_{14} \mathrm{P}_{34} \mathrm{P}_{24} \quad \text { in } \mathcal{U}(\mathcal{H} \otimes \overline{\mathcal{H}} \otimes \mathcal{H} \otimes \mathcal{H} \otimes \mathcal{H}) .
$$

The anti-Heisenberg and Heisenberg properties of our representations on the second and third leg give $\mathrm{P}_{24} \mathrm{P}_{14} \mathrm{P}_{12}=\mathrm{P}_{12} \mathrm{P}_{24}$ and $\mathrm{P}_{13} \mathrm{P}_{14} \mathrm{P}_{34}=\mathrm{P}_{34} \mathrm{P}_{13}$. Hence

$$
\mathrm{P}_{34} \mathrm{P}_{24} \mathrm{P}_{14} \mathrm{P}_{12} \mathrm{P}_{13}=\mathrm{P}_{34} \mathrm{P}_{12} \mathrm{P}_{24} \mathrm{P}_{13}=\mathrm{P}_{12} \mathrm{P}_{34} \mathrm{P}_{13} \mathrm{P}_{24}=\mathrm{P}_{12} \mathrm{P}_{13} \mathrm{P}_{14} \mathrm{P}_{34} \mathrm{P}_{24}
$$

as needed. Thus $\mathbb{U}$ and $\hat{\mathbb{V}}$ are Drinfeld compatible. The equivariance of $\mathbb{F}$ with respect to $\mathbb{U}$ in (3.7) amounts to

$$
\mathrm{P}_{25} \mathrm{P}_{15} \mathrm{P}_{45} \mathrm{P}_{35} \mathrm{P}_{14}^{*} \mathrm{P}_{24}^{*} \mathrm{~W}_{24}^{C} \mathrm{~W}_{14}^{C}=\mathrm{P}_{14}^{*} \mathrm{P}_{24}^{*} \mathrm{~W}_{24}^{C} \mathrm{~W}_{14}^{C} \mathrm{P}_{25} \mathrm{P}_{15} \mathrm{P}_{45} \mathrm{P}_{35}
$$

in $\mathcal{U}(\overline{\mathcal{H}} \otimes \mathcal{H} \otimes \overline{\mathcal{H}} \otimes \mathcal{H} \otimes \mathcal{H})$. Since we have a Heisenberg pair on the fourth leg, we may use the conditions in Proposition 2.3 to simplify

$$
\mathrm{W}_{24}^{C} \mathrm{~W}_{14}^{C} \mathrm{P}_{25} \mathrm{P}_{15} \mathrm{P}_{45}=\mathrm{W}_{24}^{C} \mathrm{P}_{25}\left(\mathrm{~W}_{14}^{C} \mathrm{P}_{15} \mathrm{P}_{45}\right)=\mathrm{W}_{24}^{C} \mathrm{P}_{25} \mathrm{P}_{45} \mathrm{~W}_{14}^{C}=\mathrm{P}_{45} \mathrm{~W}_{24}^{C} \mathrm{~W}_{14}^{C} .
$$

Hence the right side in (3.38) becomes

$$
\mathrm{P}_{14}^{*} \mathrm{P}_{24}^{*} \mathrm{~W}_{24}^{C} \mathrm{~W}_{14}^{C} \mathrm{P}_{25} \mathrm{P}_{15} \mathrm{P}_{45} \mathrm{P}_{35}=\mathrm{P}_{14}^{*} \mathrm{P}_{24}^{*} \mathrm{P}_{45} \mathrm{~W}_{24}^{C} \mathrm{~W}_{14}^{C} \mathrm{P}_{35} \text {. }
$$

Plugging this in and cancelling $\mathrm{W}_{24}^{C} \mathrm{~W}_{14}^{C} \mathrm{P}_{35}$, we see that (3.38) is equivalent to

$$
\mathrm{P}_{25} \mathrm{P}_{15} \mathrm{P}_{45} \mathrm{P}_{14}^{*} \mathrm{P}_{24}^{*}=\mathrm{P}_{14}^{*} \mathrm{P}_{24}^{*} \mathrm{P}_{45} \quad \text { or } \quad \mathrm{P}_{24} \mathrm{P}_{25} \mathrm{P}_{14} \mathrm{P}_{15} \mathrm{P}_{45}=\mathrm{P}_{45} \mathrm{P}_{24} \mathrm{P}_{14} \text {. }
$$


Since we have Heisenberg pairs on the second and fourth legs, this follows by two applications of the pentagon equation for $\mathrm{P}$.

The condition (3.8) about $\mathbb{F}$ being equivariant with respect to $\hat{\mathbb{V}}$ becomes

$$
\mathrm{P}_{14} \mathrm{P}_{15} \mathrm{P}_{12} \mathrm{P}_{13} \mathrm{P}_{25}^{*} \mathrm{P}_{35}^{*} \mathrm{~W}_{35}^{C} \mathrm{~W}_{25}^{C}=\mathrm{P}_{25}^{*} \mathrm{P}_{35}^{*} \mathrm{~W}_{35}^{C} \mathrm{~W}_{25}^{C} \mathrm{P}_{14} \mathrm{P}_{15} \mathrm{P}_{12} \mathrm{P}_{13}
$$

in $\mathcal{U}(\mathcal{H} \otimes \overline{\mathcal{H}} \otimes \mathcal{H} \otimes \overline{\mathcal{H}} \otimes \mathcal{H})$. Since we have an anti-Heisenberg pair on the second and a Heisenberg pair on the third leg, we get $\mathrm{W}_{25}^{C} \mathrm{P}_{15} \mathrm{P}_{12}=\mathrm{P}_{12} \mathrm{~W}_{25}^{C}$ and $\mathrm{P}_{13} \mathrm{P}_{15} \mathrm{~W}_{35}^{C}=$ $\mathrm{W}_{35}^{C} \mathrm{P}_{13}$. Thus the right side of (3.39) becomes

$$
\begin{aligned}
& \mathrm{P}_{25}^{*} \mathrm{P}_{35}^{*} \mathrm{~W}_{35}^{C} \mathrm{~W}_{25}^{C} \mathrm{P}_{14} \mathrm{P}_{15} \mathrm{P}_{12} \mathrm{P}_{13} \\
= & \mathrm{P}_{14} \mathrm{P}_{25}^{*} \mathrm{P}_{35}^{*} \mathrm{~W}_{35}^{C}\left(\mathrm{~W}_{25}^{C} \mathrm{P}_{15} \mathrm{P}_{12}\right) \mathrm{P}_{13}=\mathrm{P}_{14} \mathrm{P}_{25}^{*} \mathrm{P}_{35}^{*} \mathrm{~W}_{35}^{C} \mathrm{P}_{12} \mathrm{~W}_{25}^{C} \mathrm{P}_{13} \\
= & \mathrm{P}_{14} \mathrm{P}_{25}^{*} \mathrm{P}_{35}^{*} \mathrm{P}_{12}\left(\mathrm{~W}_{35}^{C} \mathrm{P}_{13}\right) \mathrm{W}_{25}^{C}=\mathrm{P}_{14} \mathrm{P}_{25}^{*} \mathrm{P}_{35}^{*} \mathrm{P}_{12} \mathrm{P}_{13} \mathrm{P}_{15} \mathrm{~W}_{35}^{C} \mathrm{~W}_{25}^{C} .
\end{aligned}
$$

Now we may cancel $\mathrm{P}_{14}$ on the left and $\mathrm{W}_{35}^{C} \mathrm{~W}_{25}^{C}$ on the right to transform our condition into $\mathrm{P}_{15} \mathrm{P}_{12} \mathrm{P}_{13} \mathrm{P}_{25}^{*} \mathrm{P}_{35}^{*}=\mathrm{P}_{25}^{*} \mathrm{P}_{35}^{*} \mathrm{P}_{12} \mathrm{P}_{13} \mathrm{P}_{15}$ or, equivalently,

$$
\mathrm{P}_{35} \mathrm{P}_{25} \mathrm{P}_{15} \mathrm{P}_{12} \mathrm{P}_{13}=\mathrm{P}_{12} \mathrm{P}_{13} \mathrm{P}_{15} \mathrm{P}_{35} \mathrm{P}_{25}
$$

in $\mathcal{U}(\mathcal{H} \otimes \overline{\mathcal{H}} \otimes \mathcal{H} \otimes \overline{\mathcal{H}} \otimes \mathcal{H})$. The anti-Heisenberg pair condition on the second leg gives $\mathrm{P}_{25} \mathrm{P}_{15} \mathrm{P}_{12}=\mathrm{P}_{12} \mathrm{P}_{25}$, the Heisenberg pair on the third leg gives $\mathrm{P}_{13} \mathrm{P}_{15} \mathrm{P}_{35}=$ $\mathrm{P}_{35} \mathrm{P}_{13}$. Plugging this in, our condition becomes

$$
\mathrm{P}_{35} \mathrm{P}_{12} \mathrm{P}_{25} \mathrm{P}_{13}=\mathrm{P}_{12} \mathrm{P}_{35} \mathrm{P}_{13} \mathrm{P}_{25},
$$

which is manifestly true. Thus our operators satisfy (3.8) as well.

Checking the braided pentagon equation (3.9) is a long computation. We may omit it because of the following trick. In the proof of Theorem [3.26, the braided pentagon equation is used exactly once. Therefore, if all the other conditions in Definition 3.2 hold, then the braided pentagon equation (3.9) is both sufficient and necessary for the usual pentagon equation for the unitary $\mathbb{W}^{D}$ constructed in Theorem 3.26. Thus the proof of Theorem 3.37 is finished up to the braided pentagon equation, and this follows from the proof of the following theorem.

Theorem 3.40. Let $\mathbb{W}^{C}, \mathbb{P} \in \mathcal{U}(\mathcal{H} \otimes \mathcal{H})$ define a $\mathrm{C}^{*}$-quantum group with projection as in Proposition 2.3. Construct a braided multiplicative unitary $(\mathbb{P}, \mathbb{U}, \hat{\mathbb{V}}, \mathbb{F})$ on the Hilbert space $\mathcal{L}=\overline{\mathcal{H}} \otimes \mathcal{H}$ with the unitaries defined in (3.34)-(3.36). From this, construct a multiplicative unitary $\mathbb{W}^{D}$ with a projection $\mathbb{P}^{D}$ on the Hilbert space

$$
\mathcal{H} \otimes \mathcal{L} \otimes \mathcal{H} \otimes \mathcal{L} \cong \mathcal{H} \otimes \overline{\mathcal{H}} \otimes \mathcal{H} \otimes \mathcal{H} \otimes \overline{\mathcal{H}} \otimes \mathcal{H}
$$

\section{by Theorem 3.26 .}

The braided multiplicative unitary $(\mathbb{P}, \mathbb{U}, \hat{\mathbb{V}}, \mathbb{F})$ and the multiplicative unitary $\mathbb{W}^{D}$ are manageable. And $\mathbb{W}^{D}$ generates the same $\mathrm{C}^{*}$-quantum group as $\mathbb{W}^{C}$. The isomorphism between these $\mathrm{C}^{*}$-quantum groups maps $\mathbb{P}$ to $\mathbb{P}^{D}$.

Roughly speaking, going from a $\mathrm{C}^{*}$-quantum group with projection to a braided $\mathrm{C}^{*}$-quantum group and back gives an isomorphic $\mathrm{C}^{*}$-quantum group with projection.

The definitions in Theorem 3.26 amount to

$$
\begin{aligned}
\mathbb{W}^{D} & :=\mathrm{P}_{14} \mathrm{P}_{34} \mathrm{P}_{24} \mathrm{P}_{46}^{*} \mathrm{P}_{45}^{*} \mathrm{P}_{26}^{*} \mathrm{P}_{36}^{*} \mathrm{~W}_{36}^{C} \mathrm{~W}_{26}^{C} \mathrm{P}_{45} \mathrm{P}_{46} \\
& =\mathrm{P}_{14} \mathrm{P}_{34} \mathrm{P}_{24} \mathrm{P}_{46}^{*} \mathrm{P}_{26}^{*} \mathrm{P}_{36}^{*} \mathrm{~W}_{36}^{C} \mathrm{~W}_{26}^{C} \mathrm{P}_{46}, \\
\mathbb{P}^{D} & :=\mathrm{P}_{14} \mathrm{P}_{34} \mathrm{P}_{24} .
\end{aligned}
$$

Our first task is to construct representations $\pi$ and $\hat{\pi}$ of $C$ and $\hat{C}$ that form a Heisenberg pair and that satisfy $(\hat{\pi} \otimes \pi) \mathbb{W}^{C}=\mathbb{W}^{D}$. This implies that $\mathbb{W}^{D}$ satisfies the pentagon equation. As we remarked above, this implies the braided pentagon equation (3.9) for $\mathbb{F}$, which still remained to be proven. 
Lemma 3.41. There is a representation $\pi^{\prime}: C \rightarrow \mathbb{B}(\mathcal{H} \otimes \overline{\mathcal{H}} \otimes \mathcal{H})$ such that

$$
\begin{aligned}
\left(\operatorname{id}_{\hat{C}} \otimes \pi^{\prime}\right) \mathrm{W}^{C} & =\mathrm{P}_{12} \mathrm{~W}_{14}^{C} & \text { in } \mathcal{U}(\hat{C} \otimes \mathbb{K}(\mathcal{H} \otimes \overline{\mathcal{H}} \otimes \mathcal{H})), \\
\left(\operatorname{id}_{\hat{C}} \otimes \pi^{\prime}\right) \mathrm{P} & =\mathrm{P}_{12} \mathrm{P}_{14} & \text { in } \mathcal{U}(\hat{C} \otimes \mathbb{K}(\mathcal{H} \otimes \overline{\mathcal{H}} \otimes \mathcal{H})) .
\end{aligned}
$$

Proof. Let $\hat{\mathrm{P}}=\sigma(\mathrm{P})^{*} \in \mathcal{U}(C \otimes \hat{C})$ for the flip $\sigma$. Define $\varphi: C \rightarrow \mathcal{M}(C \otimes \mathbb{K}(\overline{\mathcal{H}}))$ by $\varphi(c):=\hat{\mathrm{P}}_{1 \hat{\alpha}}(1 \otimes \alpha(c)) \hat{\mathrm{P}}_{1 \hat{\alpha}}^{*}$. Then

$$
\left(\operatorname{id}_{\hat{C}} \otimes \varphi\right) \mathrm{W}^{C}=\hat{\mathrm{P}}_{2 \hat{\alpha}} \mathrm{W}_{1 \alpha}^{C} \hat{\mathrm{P}}_{2 \hat{\alpha}}^{*}=\sigma_{23}\left(\mathrm{P}_{\hat{\alpha} 3}^{*} \mathrm{~W}_{1 \alpha}^{C} \mathrm{P}_{\hat{\alpha} 3}\right) \quad \text { in } \mathcal{M}(\hat{C} \otimes C \otimes \mathbb{K}(\overline{\mathcal{H}})) .
$$

The second condition in Proposition 2.3 is equivalent to $\mathrm{W}_{1 \alpha}^{C} \mathrm{P}_{\hat{\alpha} 3}=\mathrm{P}_{\hat{\alpha} 3} \mathrm{P}_{13} \mathrm{~W}_{1 \alpha}^{C}$ in $\mathcal{U}(\hat{C} \otimes \mathbb{K}(\overline{\mathcal{H}}) \otimes C)$ because we have an anti-Heisenberg pair on the second leg. Thus

$$
\left(\operatorname{id}_{\hat{C}} \otimes \varphi\right) \mathrm{W}^{C}=\sigma_{23}\left(\mathrm{P}_{13} \mathrm{~W}_{1 \alpha}^{C}\right)=\mathrm{P}_{12} \mathrm{~W}_{1 \alpha}^{C} \quad \text { in } \mathcal{M}(\hat{C} \otimes C \otimes \mathbb{K}(\overline{\mathcal{H}})) .
$$

We may define $\pi^{\prime}(c):=\left(\left(\hat{\iota} \otimes \iota \circ \alpha^{-1}\right) \varphi(c)\right)_{13}$ because $\alpha$ is automatically injective (see [18, Proposition 3.7]). This is the unique representation that satisfies (3.42). Replacing $\mathrm{W}^{C}$ by $\mathrm{P}$ in the above computations gives (3.43).

Lemma 3.44. Let $\pi^{\prime}$ be as in the previous lemma. The pair of representations $(\pi, \hat{\pi})$ of $C$ and $\hat{C}$ on $\mathcal{H} \otimes \overline{\mathcal{H}} \otimes \mathcal{H}$ defined by

$$
\pi(c):=\mathrm{P}_{13}^{*} \pi^{\prime}(c) \mathrm{P}_{13}, \quad \hat{\pi}(\hat{c}):=\mathrm{P}_{13}^{*}\left((\hat{\alpha} \otimes \hat{\imath}) \hat{\Delta}_{C}(\hat{c})\right)_{23} \mathrm{P}_{13}
$$

is an $\mathbb{H}$-Heisenberg pair.

Proof. Let $\hat{\pi}^{\prime}(\hat{c}):=\left((\hat{\alpha} \otimes \hat{\imath}) \hat{\Delta}_{C}(\hat{c})\right)_{23}$. The lemma is equivalent to $\left(\pi^{\prime}, \hat{\pi}^{\prime}\right)$ being $\mathbb{H}$-Heisenberg. Recall that $\left(\hat{\Delta}_{C} \otimes \mathrm{id}_{C}\right) \mathrm{W}^{C}=\mathrm{W}_{23}^{C} \mathrm{~W}_{13}^{C}$. Lemma 3.41 gives

$$
\mathrm{W}_{\tilde{\pi}^{\prime} 5}^{C} \mathrm{~W}_{1 \pi^{\prime}}^{C}=\mathrm{W}_{45}^{C} \mathrm{~W}_{35}^{C} \mathrm{P}_{12} \mathrm{~W}_{14}^{C}=\mathrm{P}_{12} \mathrm{~W}_{45}^{C} \mathrm{~W}_{14}^{C} \mathrm{~W}_{35}^{C}
$$

in $\mathcal{U}(\hat{C} \otimes \mathbb{K}(\mathcal{H} \otimes \overline{\mathcal{H}} \otimes \mathcal{H}) \otimes C)$. Since we have a Heisenberg pair on the fourth leg, the pentagon equation (3.3) gives

$$
\mathrm{P}_{12} \mathrm{~W}_{45}^{C} \mathrm{~W}_{14}^{C} \mathrm{~W}_{35}^{C}=\mathrm{P}_{12} \mathrm{~W}_{14}^{C} \mathrm{~W}_{15}^{C} \mathrm{~W}_{45}^{C} \mathrm{~W}_{35}^{C} \text {, }
$$

which is equivalent to $\mathrm{W}_{\hat{\pi}^{\prime} 5}^{C} \mathrm{~W}_{1 \pi^{\prime}}^{C}=\mathrm{W}_{1 \pi^{\prime}}^{C} \mathrm{~W}_{15}^{C} \mathrm{~W}_{\hat{\pi}^{\prime} 5}^{C}$ in $\mathcal{U}(\hat{C} \otimes \mathbb{K}(\mathcal{H} \otimes \overline{\mathcal{H}} \otimes \mathcal{H}) \otimes C)$.

Lemma 3.45. $\mathbb{W}^{D}=(\hat{\pi} \otimes \pi) \mathrm{W}^{C}$ and $\mathbb{P}^{D}=(\hat{\pi} \otimes \pi) \mathrm{P}$ in $\mathcal{U}(\mathcal{H} \otimes \mathcal{L} \otimes \mathcal{H} \otimes \mathcal{L})$.

Proof. Cancelling $\mathrm{P}_{45}$ gives $\mathbb{W}^{D}=\mathrm{P}_{14} \mathrm{P}_{34} \mathrm{P}_{24} \mathrm{P}_{46}^{*} \mathrm{P}_{26}^{*} \mathrm{P}_{36}^{*} \mathrm{~W}_{36}^{C} \mathrm{~W}_{26}^{C} \mathrm{P}_{46}$. Computing as in the proof of Lemma 3.44, we get

$$
(\hat{\pi} \otimes \pi) \mathrm{W}^{C}=\mathrm{P}_{13}^{*} \mathrm{P}_{46}^{*} \mathrm{P}_{34} \mathrm{~W}_{36}^{C} \mathrm{P}_{24} \mathrm{~W}_{26}^{C} \mathrm{P}_{13} \mathrm{P}_{46}=\mathrm{P}_{46}^{*} \mathrm{P}_{13}^{*} \mathrm{P}_{34} \mathrm{P}_{24} \mathrm{~W}_{36}^{C} \mathrm{P}_{13} \mathrm{~W}_{26}^{C} \mathrm{P}_{46} .
$$

Since we have a Heisenberg pair on the third leg, $\mathrm{W}_{36}^{C} \mathrm{P}_{13}=\mathrm{P}_{13} \mathrm{P}_{16} \mathrm{~W}_{36}^{C}$. Hence

$$
(\hat{\pi} \otimes \pi) \mathrm{W}^{C}=\mathrm{P}_{46}^{*} \mathrm{P}_{13}^{*} \mathrm{P}_{34} \mathrm{P}_{24} \mathrm{P}_{13} \mathrm{~W}_{36}^{C} \mathrm{~W}_{26}^{C} \mathrm{P}_{46} .
$$

Since we have Heisenberg pairs on the third and fourth legs, $\mathrm{P}_{34} \mathrm{P}_{13}=\mathrm{P}_{13} \mathrm{P}_{14} \mathrm{P}_{34}$ and $\mathrm{P}_{46} \mathrm{P}_{i 4}=\mathrm{P}_{i 6} \mathrm{P}_{i 6} \mathrm{P}_{46}$ for all $i=1,2,3$. Using these identities (for the expressions within brackets in the computation below) we get

$$
\begin{aligned}
& \mathrm{P}_{46}^{*} \mathrm{P}_{13}^{*} \mathrm{P}_{34} \mathrm{P}_{24} \mathrm{P}_{13} \mathrm{P}_{16}=\mathrm{P}_{46}^{*}\left(\mathrm{P}_{13}^{*} \mathrm{P}_{34} \mathrm{P}_{13}\right) \mathrm{P}_{24} \mathrm{P}_{16}=\mathrm{P}_{46}^{*} \mathrm{P}_{14} \mathrm{P}_{34} \mathrm{P}_{24} \mathrm{P}_{16} \\
&=\left(\mathrm{P}_{46}^{*} \mathrm{P}_{14} \mathrm{P}_{16}\right) \mathrm{P}_{34} \mathrm{P}_{24}=\mathrm{P}_{14}\left(\mathrm{P}_{46}^{*} \mathrm{P}_{34}\right) \mathrm{P}_{24}=\mathrm{P}_{14} \mathrm{P}_{34} \mathrm{P}_{46}^{*} \mathrm{P}_{36}^{*} \mathrm{P}_{24} \\
&=\mathrm{P}_{14} \mathrm{P}_{34}\left(\mathrm{P}_{46}^{*} \mathrm{P}_{24}\right) \mathrm{P}_{36}^{*}=\mathrm{P}_{14} \mathrm{P}_{34} \mathrm{P}_{24} \mathrm{P}_{46}^{*} \mathrm{P}_{26}^{*} \mathrm{P}_{36}^{*} .
\end{aligned}
$$

The last two computations together give

$$
(\hat{\pi} \otimes \pi) \mathrm{W}^{C}=\mathrm{P}_{14} \mathrm{P}_{34} \mathrm{P}_{24} \mathrm{P}_{46}^{*} \mathrm{P}_{26}^{*} \mathrm{P}_{36}^{*} \mathrm{~W}_{36}^{C} \mathrm{~W}_{26}^{C} \mathrm{P}_{46}=\mathbb{W}^{D} .
$$

Equation (3.43) allows a similar computation with $\mathrm{P}$ instead of $\mathrm{W}^{C}$. This gives

$$
(\hat{\pi} \otimes \pi) \mathrm{P}=\mathrm{P}_{14} \mathrm{P}_{34} \mathrm{P}_{24} \mathrm{P}_{46}^{*} \mathrm{P}_{26}^{*} \mathrm{P}_{36}^{*} \mathrm{P}_{36} \mathrm{P}_{26} \mathrm{P}_{46}=\mathbb{P}^{D} .
$$


The following remarks apply to any Heisenberg pair $(\pi, \hat{\pi})$ for a $\mathrm{C}^{*}$-quantum group $\mathbb{H}=\left(C, \Delta_{C}\right)$ on a Hilbert space $\mathcal{H}^{\prime}$. Being a Heisenberg pair means that $(\hat{\pi} \otimes \pi) \mathrm{W}^{C}$ is a multiplicative unitary. It is unclear, in general, whether this multiplicative unitary is manageable. If it is manageable, then we claim that the $\mathrm{C}^{*}$-quantum group that it generates is isomorphic to the one we started with. The representations in a Heisenberg pair are automatically faithful by [18, Proposition 3.7]. Hence we may view $C$ and $\hat{C}$ as subalgebras of $\mathbb{B}\left(\mathcal{H}^{\prime}\right)$, and $(\hat{\pi} \otimes \pi) \mathrm{W}^{C}$ is a unitary multiplier of $\hat{C} \otimes C \subseteq \mathbb{B}\left(\mathcal{H}^{\prime} \otimes \mathcal{H}^{\prime}\right)$. It makes no difference whether we take slices on the first leg with elements of $\mathbb{B}\left(\mathcal{H}^{\prime}\right)_{*}$ or $\hat{C}^{*}$ : both generate the same $\mathrm{C}^{*}$-subalgebra of $\mathbb{B}\left(\mathcal{H}^{\prime}\right)$, namely, $\pi(C)$. The comultiplication on the quantum group generated by $(\hat{\pi} \otimes \pi) \mathrm{W}^{C}$ is defined so that the isomorphism $\pi$ is a Hopf *-homomorphism. Thus the $\mathrm{C}^{*}$-quantum group generated by $(\hat{\pi} \otimes \pi) \mathrm{W}^{C}$ is isomorphic to $\mathbb{H}$ for any Heisenberg pair for which $(\hat{\pi} \otimes \pi) \mathrm{W}^{C}$ is manageable. Furthermore, Lemma 3.45 shows that this Hopf *-isomorphism maps $\mathrm{P}$ to $\mathbb{P}^{D}$, so we also get the same projection on our $\mathrm{C}^{*}$-quantum group.

Thus the proof of Theorem 3.40 will be finished once we show that $\mathbb{W}^{D}$ and the braided multiplicative unitary $(\mathbb{U}, \hat{\mathbb{V}}, \mathbb{F})$ are manageable. By Theorem $3.29, \mathbb{W}^{D}$ is manageable once $(\mathbb{U}, \hat{\mathbb{V}}, \mathbb{F})$ is manageable. So it remains to prove this.

The braiding on $\mathcal{L} \otimes \mathcal{L}$ comes from the unique unitary $Z$ that verifies (3.10). A simple computation shows that $Z=\mathrm{P}_{14}^{*} \mathrm{P}_{24}^{*} \mathrm{P}_{13}^{*} \mathrm{P}_{23}^{*}$ in $\mathcal{U}(\overline{\mathcal{H}} \otimes \mathcal{H} \otimes \overline{\mathcal{H}} \otimes \mathcal{H})$ does the job. This gives

$$
Z^{*} \mathbb{F}=\mathrm{P}_{23} \mathrm{P}_{13} \mathrm{P}_{24} \mathrm{P}_{14} \mathrm{P}_{14}^{*} \mathrm{P}_{24}^{*} \mathrm{~W}_{24}^{C} \mathrm{~W}_{14}^{C}=\mathrm{P}_{23} \mathrm{P}_{13} \mathrm{~W}_{24}^{C} \mathrm{~W}_{14}^{C} .
$$

Now we use that $(\iota, \hat{\imath})$ is the standard Heisenberg pair, generated by $\mathbb{W}^{C}$, and that the anti-Heisenberg pair $(\alpha, \hat{\alpha})$ is constructed as in [11, Lemma 3.6]; that is,

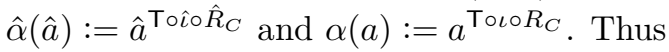

$$
Z^{*} \mathbb{F}=\mathrm{P}_{23}^{\hat{\imath} \otimes \mathrm{T} \circ \iota \circ R_{C}} \mathrm{P}_{13}^{\mathrm{T} \circ \hat{\imath} \circ \hat{R}_{C} \otimes \mathrm{T} \circ \iota \circ R_{C}}\left(\mathrm{~W}_{24}^{C}\right)^{\hat{\imath} \otimes \iota}\left(\mathrm{W}_{14}^{C}\right)^{\mathrm{T} \circ \hat{\imath} \circ \hat{R}_{C} \otimes \iota} .
$$

Let $Q_{C}$ and $\widetilde{\mathbb{W}^{C}} \in \mathcal{U}(\overline{\mathcal{H}} \otimes \mathcal{H})$ witness the manageability of $\mathbb{W}^{C}=(\hat{\imath} \otimes \iota) \mathrm{W}^{C} \in$ $\mathcal{U}(\mathcal{H} \otimes \mathcal{H})$, see Appendix A Since $\mathbb{P}$ is manageable by Proposition 2.5, so is the dual $\widehat{\mathbb{P}}=\Sigma \mathbb{P}^{*} \Sigma$. This is witnessed by a certain unitary $\widetilde{\mathbb{P}} \in \mathcal{U}(\overline{\mathcal{H}} \otimes \mathcal{H})$. We have $\left(\mathrm{W}^{C}\right)^{\mathrm{T} \hat{\imath} R_{\hat{C}} \otimes \iota}=\left(\mathrm{W}^{C}\right)^{\mathrm{T} \hat{\imath} \otimes \iota R_{C}}=\left(\widetilde{\mathbb{W}^{C}}\right)^{*}$ and $\mathrm{P}^{\mathrm{T} \hat{\imath} R_{\hat{C}} \otimes \mathrm{T} \iota R_{C}}=\mathrm{P}^{\mathrm{T} \otimes \mathrm{T}}$ by [28, Theorem $1.6(5)]$ and [10, (19)]. Similarly, $\left(\Sigma \mathrm{P}^{\hat{\imath} \otimes T \circ \iota \circ R_{C} \Sigma}\right)^{*}=\hat{\mathrm{P}}^{\mathrm{T} \circ\left\llcorner\circ R_{C} \otimes \hat{\imath}\right.}=\widetilde{\widehat{\mathbb{P}}}$. Thus

$$
Z^{*} \mathbb{F}=\Sigma_{23} \widetilde{\widehat{\mathbb{P}}}_{23} \Sigma_{23} \mathbb{P}_{13}^{\mathbf{T} \otimes \top} \mathbb{W}_{24}^{C}\left(\widetilde{\mathbb{W}}_{14}^{C}\right)^{*} \quad \text { in } \mathcal{U}(\overline{\mathcal{H}} \otimes \mathcal{H} \otimes \overline{\mathcal{H}} \otimes \mathcal{H})
$$

Let $Q:=Q_{C}^{\top} \otimes Q_{C}$. Then $Q \otimes Q$ commutes with $\mathbb{F}, Q \otimes Q_{C}$ commutes with $\mathbb{U}$, and $Q_{C} \otimes Q$ commutes with $\hat{\mathbb{V}}$. Define $\widetilde{\mathbb{F}} \in \mathcal{U}(\overline{\mathcal{L}} \otimes \mathcal{L})$ by

$$
\widetilde{\mathbb{F}}:=\widetilde{\mathbb{W}}_{24}^{C}\left(\mathbb{W}_{14}^{C}\right)^{*}\left(\mathbb{P}^{*}\right)_{23}^{\mathrm{T} \otimes \mathrm{T}} \widetilde{\mathbb{P}}_{13}^{\top} \otimes \mathrm{T} \quad \text { in } \mathcal{U}(\mathcal{H} \otimes \overline{\mathcal{H}} \otimes \overline{\mathcal{H}} \otimes \mathcal{H}) .
$$

This unitary and $Q$ witness the manageability of the braided multiplicative unitary $(\mathbb{U}, \hat{\mathbb{V}}, \mathbb{F})$. The rather technical proof of this fact is relegated to the appendix, see Lemma A.8.

\section{Examples of Quantum Groups with Projections}

The simplest examples of semidirect products of connected Lie groups are $\mathrm{E}(2)=$ $\mathbb{R}^{2} \rtimes \mathbb{T}$ and the real and complex $a x+b$-groups $\mathbb{R} \rtimes \mathbb{R}_{>0}^{\times}$and $\mathbb{C} \rtimes \mathbb{C}^{\times}$, where the second, multiplicative factor acts by multiplication on the first, additive factor. The group $\mathrm{E}(2)$ is the group of isometries of the plane. Another very important example is the Poincaré group, the semidirect product of the Lorentz group with $\mathbb{R}^{4}$.

When quantising such groups, one may try to preserve the semidirect product structure, that is, construct $\mathrm{C}^{*}$-quantum groups with projection. For instance, the 
quantum E(2) groups by Woronowicz 26] have obvious morphisms to the circle group $\mathbb{T}$ and back that compose to the identity on $\mathbb{T}$. The quantum $a z+b$ groups introduced by Woronowicz 29] and Soltan [20] - which deform $\mathbb{C} \rtimes \mathbb{C}^{\times}$- have obvious morphisms to the group $\mathbb{C}_{q}^{\times}=q^{\mathbb{Z}+\mathrm{i} \mathbb{R}} \subseteq \mathbb{C}^{\times}$(with multiplication as group structure) and back, which compose to the identity on $\mathbb{C}_{q}^{\times}$; see also [6, Example 3.7]. The quantum $a x+b$ group by Woronowicz and Zakrzewski [31] has an obvious projection onto the group $\mathbb{R}_{>0}^{\times} \cong \mathbb{R}$.

There are also quantum versions of semidirect product groups that appear to have no such projection. This includes the $a z+b$ groups by Baaj and Skandalis, see [25. Section 5.3], the $a x+b$ groups by Stachura [23] and the $\kappa$-Poincaré groups by Stachura 22. These examples are all constructed using the formalism of quantum group extensions of [25]. Quantum group extensions are compared with quantum groups with projection in 7 .

As an example of our theory, we are going to construct a braided multiplicative unitary that generates "simplified quantum E(2)," a variant of quantum E(2) also due to Woronowicz (unpublished); whereas the quantum E(2) groups in [26] deform a double cover of $\mathrm{E}(2)$, the simplified variants deform $\mathrm{E}(2)$ itself. A common feature of simplified quantum $\mathrm{E}(2)$ and the quantum groups with projection mentioned above is that the image of the projection is a classical, Abelian group. This is to be expected when deforming semidirect products by Abelian groups because these cannot be deformed to quantum groups in interesting ways. We begin by observing some common features of braided multiplicative unitaries in case $\mathbb{W}$ generates an Abelian group $G$.

Let $\hat{G}$ be the dual group. The corepresentations $\mathbb{U}$ and $\hat{\mathbb{V}}$ in a braided multiplicative unitary are equivalent to representations of $G$ and $\hat{G}$ on the Hilbert space $\mathcal{L}$, respectively. The compatibility condition (3.6) for $\mathbb{U}$ and $\hat{\mathbb{V}}$ says here that the representations of $G$ and $\hat{G}$ commute. Thus we may combine them to one representation of $\hat{G} \times G$ on $\mathcal{L}$.

We can further normalise this representation because the left regular representation of any quantum group absorbs every other representation. The operator $\mathbb{F} \otimes 1$ on $\mathcal{L} \otimes L^{2}(\hat{G} \times G)$ is a braided multiplicative unitary if and only if $\mathbb{F}$ is, and it generates an equivalent semidirect product quantum group. Thus we may assume without loss of generality that our representation is a multiple of the left regular representation:

$$
\mathcal{L}=L^{2}(\hat{G} \times G) \otimes \mathcal{L}_{0}
$$

for some separable Hilbert space $\mathcal{L}_{0}$, with $\mathrm{C}^{*}(\hat{G} \times G)$ acting only on the first tensor factor, by the regular representation. We may identify $\mathrm{C}_{0}(G \times \hat{G}) \cong \mathrm{C}^{*}(\hat{G} \times G)$ and $L^{2}(\hat{G} \times G) \cong L^{2}(G \times \hat{G})$ by the Fourier transform, and the regular representation of $\mathrm{C}^{*}(\hat{G} \times G)$ on $L^{2}(\hat{G} \times G) \cong L^{2}(G \times \hat{G})$ becomes the standard representation of $\mathrm{C}_{0}(G \times \hat{G})$ on $L^{2}(G \times \hat{G})$ by pointwise multiplication.

For some examples, a variant of the above is useful: if the representation of $\hat{G} \times G$ on $\mathcal{L}$ factors through an Abelian locally compact group $H$, then we may use $H$ instead of $\hat{G} \times G$ in the above simplification. That is, we seek a braided multiplicative unitary on the Hilbert space $L^{2}(H) \otimes \mathcal{L}_{0}$ with $\hat{G} \times G$ acting only on the first tensor factor, through the regular representation of $H$ and the given homomorphism $\hat{G} \times G \rightarrow H$.

For instance, the compact quantum group $\mathrm{U}_{q}(2)$ is a semidirect product of the braided quantum group $\mathrm{SU}_{q}(2)$ by the circle $\mathbb{T}$ (see [5]), and the relevant representation of $\mathbb{Z} \times \mathbb{T}$ factors through a homomorphism $\mathbb{Z} \times \mathbb{T} \rightarrow \mathbb{T},(n, z) \mapsto \lambda^{n} \cdot z$ for some $\lambda \in \mathbb{T}$. For the quantum $a z+b$ groups, $G=\mathbb{C}_{q}$ is self-dual, and the relevant 
representation of $\hat{G} \times G$ factors through the map $\hat{G} \times G \cong G \times G \rightarrow G$, where the second map is the multiplication map $(x, y) \mapsto x \cdot y$.

When we have simplified $\mathcal{L}$ to $L^{2}(G \times \hat{G}) \otimes \mathcal{L}_{0}$ with $\mathrm{C}_{0}(G \times \hat{G})$ acting by pointwise multiplication, the braiding operator $\mathcal{L}_{\chi^{\mathcal{L}}}$ is the operator of pointwise multiplication with the circle-valued function

$$
\left(g_{1}, \chi_{1}, g_{2}, \chi_{2}\right) \mapsto \chi_{1}\left(g_{2}\right) .
$$

The conditions (3.7) and (3.8) for $\mathbb{F}$ mean that $\mathbb{F} \in \mathcal{U}(\mathcal{L} \otimes \mathcal{L})$ is a $\hat{G} \times G$-equivariant operator with respect to the tensor product representation of $\hat{G} \times G$ on $\mathcal{L} \otimes \mathcal{L}$. In terms of the above spectral analysis, $f \in \mathrm{C}_{0}(G \times \hat{G})$ acts on $\mathcal{L} \otimes \mathcal{L}$ by pointwise multiplication on each fibre with the function

$$
\Delta^{*} f\left(g_{1}, \chi_{1}, g_{2}, \chi_{2}\right)=f\left(g_{1} g_{2}, \chi_{1} \chi_{2}\right)
$$

for all $\left(g_{1}, \chi_{1}, g_{2}, \chi_{2}\right) \in G \times \hat{G} \times G \times \hat{G}$. An operator on $\mathcal{L} \otimes \mathcal{L}$ is $\hat{G} \times G$-equivariant if and only if it commutes with the operators of pointwise multiplication by $\Delta^{*} f$ for $f \in \mathrm{C}_{0}(G \times \hat{G})$.

Summing up, it suffices to look for braided multiplicative unitaries over an Abelian group $G$ on the Hilbert space $\mathcal{L}=L^{2}\left(G \times \hat{G}, \ell^{2}(\mathbb{N})\right)$. Such a braided multiplicative unitary $\mathbb{F} \in \mathcal{U}(\mathcal{L} \otimes \mathcal{L})$ must commute with the operators of pointwise multiplication by functions in $\Delta^{*} \mathrm{C}_{\mathrm{b}}(G \times \hat{G}) \subseteq \mathrm{C}_{\mathrm{b}}(G \times \hat{G} \times G \times \hat{G})$, and it must satisfy the braided pentagon equation (3.9), where the braiding is given by pointwise multiplication with the function in (4.1).

4.1. Simplified quantum E(2) groups. Now we specialise to simplified quantum E(2) groups. They were already treated in [17] except for the manageability of the braided multiplicative unitary in question. A $\mathrm{C}^{*}$-algebraic version of this construction appears in [19].

Here the image of the projection is the circle group $G=\mathbb{T}$, so $\hat{G}=\mathbb{Z}$. The analysis above suggests to construct a braided multiplicative unitary for quantum $\mathrm{E}(2)$ on a Hilbert space of the form $L^{2}(\mathbb{T} \times \mathbb{Z}) \otimes \mathcal{L}_{0}$. Actually, we shall not need $\mathcal{L}_{0}$ and work on $L^{2}(\mathbb{T} \times \mathbb{Z})$ itself. First we describe the standard multiplicative unitary $\mathbb{W}$ generating $\mathbb{T}$.

Let $\mathcal{H}:=\ell^{2}(\mathbb{Z})$ and let $\left\{e_{p}\right\}_{p \in \mathbb{Z}}$ be an orthonormal basis of $\mathcal{H}$. Define

$$
u e_{p}:=e_{p+1} \quad \text { and } \quad \hat{N} e_{p}:=p e_{p} .
$$

The shift $u$ is unitary and generates the regular representation of $\mathbb{Z}$ on $\mathcal{H}$. The operator $\hat{N}$ is self-adjoint with spectrum $\mathbb{Z}$, and the resulting representation of $\mathrm{C}_{0}(\mathbb{Z}) \cong \mathrm{C}^{*}(\mathbb{T})$ is the Fourier transform of the regular representation of $\mathbb{T}$ on $L^{2}(\mathbb{T})$. These operators generate a representation of the crossed product $\mathrm{C}_{0}(\mathbb{Z}) \rtimes \mathbb{Z} \cong$ $\mathbb{K}\left(\ell^{2} \mathbb{Z}\right)$. That is, they satisfy the commutation relation

$$
u^{*} \hat{N} u=\hat{N}+1 \text {. }
$$

The multiplicative unitary generating $\mathbb{T}$ is

$$
\mathbb{W}:=(1 \otimes u)^{\hat{N} \otimes 1}=\int_{\mathbb{Z} \times \mathbb{T}} z^{s} \mathrm{~d} E_{\hat{N}}(s) \otimes \mathrm{d} E_{u}(z), \quad e_{k} \otimes e_{l} \mapsto e_{k} \otimes e_{l+k},
$$

where $\mathrm{d} E_{\hat{N}}$ and $\mathrm{d} E_{u}$ are the spectral measures of $\hat{N}$ and $u$, respectively. The commutation relation (4.2) implies the pentagon equation

$$
(1 \otimes 1 \otimes u)^{1 \otimes \hat{N} \otimes 1}(1 \otimes u \otimes 1)^{\hat{N} \otimes 1 \otimes 1}\left(1 \otimes 1 \otimes u^{*}\right)^{1 \otimes \hat{N} \otimes 1}=(1 \otimes u \otimes u)^{\hat{N} \otimes 1 \otimes 1} .
$$

for $\mathbb{W}$, that is, $\mathbb{W}$ is a multiplicative unitary. Simple computations show that $Q=1$ and $\widetilde{\mathbb{W}}:=\left(1 \otimes u^{*}\right)^{\hat{N} \otimes 1}$ witness the manageability of $\mathbb{W}$. Slices of $\mathbb{W}$ in the first and second leg clearly generate the $\mathrm{C}^{*}$-algebras $\mathrm{C}^{*}(\hat{N}) \cong \mathrm{C}_{0}(\mathbb{Z})$ and 
$\mathrm{C}^{*}(u) \cong \mathrm{C}_{0}(\mathbb{T})$. The comultiplications defined by $\mathbb{W}$ satisfy $\Delta_{\mathrm{C}(\mathbb{T})}(u):=u \otimes u$ and $\Delta_{\mathrm{C}_{0}(\mathbb{Z})}(\hat{N}):=\hat{N} \otimes 1 \dot{+} 1 \otimes \hat{N}$, where $\hat{N} \otimes 1 \dot{+} 1 \otimes \hat{N}$ means the unbounded affiliated element of $\mathrm{C}_{0}(\mathbb{Z} \times \mathbb{Z})$ given by the closure of the essentially self-adjoint operator $\hat{N} \otimes 1+1 \otimes \hat{N}$.

Let $0<q<1$. We identify $\mathbb{Z} \times \mathbb{T} \cong \mathbb{C}_{q}^{\times}=q^{\mathbb{Z}+\mathrm{i} \mathbb{R}} \subseteq \mathbb{C}^{\times}$by mapping $(n, z) \mapsto q^{n} \cdot z$. As suggested above, we are going to construct a multiplicative unitary on the Hilbert space $L^{2}(\mathbb{Z} \times \mathbb{T})=L^{2}\left(\mathbb{C}_{q}^{\times}\right)$with the regular representation of $\mathbb{Z} \times \mathbb{T}$. We choose the orthonormal basis $e_{k, l}:=\delta_{k} \otimes z^{l}$ for $k, l \in \mathbb{Z}$ in $L^{2}\left(\mathbb{C}_{q}^{\times}\right)$and thus identify $\mathcal{L} \cong \mathcal{H} \otimes \mathcal{H}$. In our chosen basis, $\mathbb{Z}$ acts by $\hat{\alpha}_{n}\left(e_{k, l}\right)=e_{k+n, l}$ for $n, k, l \in \mathbb{Z}$ and $\mathbb{T}$ acts by $\alpha_{\zeta}\left(e_{k, l}\right)=\zeta^{l} \cdot e_{k, l}$ for $k, l \in \mathbb{Z}, \zeta \in \mathbb{T}$. Thus the right and left corepresentations $\mathbb{U} \in \mathcal{U}(\mathcal{L} \otimes \mathcal{H})$ and $\hat{\mathbb{V}} \in \mathcal{U}(\mathcal{H} \otimes \mathcal{L})$ and the resulting braiding operator $\mathcal{L}_{\chi^{\mathcal{L}}} \in \mathcal{U}(\mathcal{L} \otimes \mathcal{L})$ are

$$
\begin{aligned}
\mathbb{U} & =\mathbb{W}_{23}, & e_{k, l, m} & \mapsto e_{k, l, m+l} \\
\hat{\mathbb{V}} & =\mathbb{W}_{12}, & e_{m, k, l} & \mapsto e_{m, k+m, l} \\
\mathcal{L}_{\chi \mathcal{L}}=Z \Sigma & =\mathbb{W}_{23}^{*} \Sigma, & e_{k, l} \otimes e_{n, p} & \mapsto e_{n, p} \otimes e_{k-p, l} .
\end{aligned}
$$

We also describe the representations of $\mathrm{C}(\mathbb{T}) \cong \mathrm{C}^{*}(\mathbb{Z})$ and $\mathrm{C}_{0}(\mathbb{Z}) \cong \mathrm{C}^{*}(\mathbb{T})$ on $\mathcal{L}$ through a unitary operator $\mathcal{U}$ and a self-adjoint operator $\hat{\mathcal{N}}$ with spectrum $\mathbb{Z}$ and commuting with $\mathcal{U}$ :

$$
\mathcal{U}\left(e_{k, l}\right):=e_{k+1, l} \quad \hat{\mathcal{N}}\left(e_{k, l}\right):=l e_{k, l} .
$$

We define a closed operator $\Upsilon=\Phi_{\Upsilon}|\Upsilon|$ on $\mathcal{L}$ by

$$
\Phi_{\Upsilon} e_{k, l}:=e_{k, l+1}, \quad|\Upsilon| e_{k, l}:=q^{2 k+l} e_{k, l}, \quad \Upsilon e_{k, l}:=q^{2 k+l} e_{k, l+1} .
$$

The operator $\Phi_{\Upsilon}$ is unitary and $|\Upsilon|$ is strictly positive with spectrum $q^{\mathbb{Z}} \cup\{0\}$, and $\Phi_{\Upsilon}$ and $|\Upsilon|$ satisfy the following commutation relations:

$$
\left\{\begin{aligned}
\Phi_{\Upsilon}|\Upsilon| \Phi_{\Upsilon}^{*} & =q^{-1}|\Upsilon|, & & \\
\mathcal{U} \Phi_{\Upsilon} & =\Phi_{\Upsilon} \mathcal{U}, & & \mathcal{U}|\Upsilon| \mathcal{U}^{*}=q^{-2}|\Upsilon|, \\
\Phi_{\Upsilon} \hat{\mathcal{N}} \Phi_{\Upsilon}^{*} & =\hat{\mathcal{N}}-1, & & |\Upsilon| \text { and } \hat{\mathcal{N}} \text { strongly commute. }
\end{aligned}\right.
$$

Thus $\Upsilon^{-1}\left(e_{k, l}\right)=q^{-2 k-l+1} e_{k, l-1}$. The closed operator

$$
X:=\Upsilon q^{-2 \hat{\mathcal{N}}} \otimes \Upsilon^{-1}, \quad e_{k, l} \otimes e_{n, p} \mapsto q^{2(k-n)-(l+p)+1} e_{k, l+1} \otimes e_{n, p-1},
$$

on $\mathcal{L} \otimes \mathcal{L}$ is normal because $|X|: e_{k, l} \otimes e_{n, p} \mapsto q^{2(k-n)-(l+p)+1} e_{k, l} \otimes e_{n, p}$ commutes with its phase $\Phi_{X}: e_{k, l} \otimes e_{n, p} \mapsto e_{k, l+1} \otimes e_{n, p-1}$ in the polar decomposition. The spectrum of $X$ is $\overline{\mathbb{C}}_{q}:=\mathbb{C}_{q}^{\times} \cup\{0\}$. Both $|X|$ and $\Phi_{X}$ strongly commute with $\mathcal{U} \otimes \mathcal{U}$ and $\hat{\mathcal{N}} \otimes 1+1 \otimes \hat{\mathcal{N}}$. Thus $X$ is equivariant for the tensor product representations $\mathbb{U} \oplus \mathbb{U}$ and $\hat{\mathbb{V}} \oplus \hat{\mathbb{V}}$. Hence any circle-valued function $F: \overline{\mathbb{C}}_{q} \rightarrow \mathbb{T}$ gives a unitary $F(X)$ on $\mathcal{L} \otimes \mathcal{L}$ that is equivariant with respect to $\mathbb{U} \oplus \mathbb{U}$ and $\hat{\mathbb{V}} \oplus \hat{\mathbb{V}}$.

We want to choose $F$ so that $F(X)$ satisfies the braided pentagon equation. Since the functional calculus is compatible with conjugation by unitaries, the top-braided pentagon equation for $F(X)$ says

$$
\begin{aligned}
& F\left(F\left(X_{23}\right) X_{12} F\left(X_{23}\right)^{*}\right)=F\left(X_{12}\right)^{\mathcal{L}}{ }^{\mathcal{L}}{ }_{23} F\left(X_{12}\right)^{\mathcal{L}}{ }^{\mathcal{L}}{ }^{\mathcal{L}} \\
& \\
&=F\left(X_{12}\right) F\left(Z_{23} X_{13} Z_{23}^{*}\right)
\end{aligned}
$$

We compute

$$
Z_{23} X_{13} Z_{23}^{*}\left(e_{k, l} \otimes e_{n, p} \otimes e_{r, s}\right)=q^{2(k-p-r)-(l+s)+1}\left(e_{k, l+1} \otimes e_{n, p} \otimes e_{p, s-1}\right) .
$$

Thus

$$
Z_{23} X_{13} Z_{23}^{*}=\Upsilon q^{-2 \hat{\mathcal{N}}} \otimes q^{-2 \hat{\mathcal{N}}} \otimes \Upsilon^{-1}=X_{12} \cdot X_{23}
$$


Hence (4.4) becomes

$$
F\left(F\left(X_{23}\right) X_{12} F\left(X_{23}\right)\right)=F\left(X_{12}\right) F\left(X_{12} X_{23}\right) .
$$

The quantum exponential function is defined in [30] by

$$
\mathrm{F}_{q}(z):= \begin{cases}\prod_{k=1}^{\infty} \frac{1+q^{2 k} \bar{z}}{1+q^{2 k} z} & z \in \overline{\mathbb{C}}_{q} \backslash\left\{-q^{2 \mathbb{Z}}\right\} \\ -1 & \text { otherwise. }\end{cases}
$$

This product converges absolutely outside $-q^{2 \mathbb{Z}}$, and $\left|\frac{1+q^{2 k} \bar{z}}{1+q^{2 k} z}\right|=1$ for all $z \neq-q^{2 k}$. Thus $\mathrm{F}_{q}$ is a unitary multiplier of $\mathrm{C}_{0}\left(\overline{\mathbb{C}_{q}}\right)$, and

$$
\mathbb{F}:=\mathrm{F}_{q}\left(\Upsilon^{-1} q^{-2 \hat{\mathcal{N}}} \otimes \Upsilon\right)
$$

is a unitary operator on $\mathcal{L} \otimes \mathcal{L}$.

Theorem 4.7. The triple $(\mathbb{U}, \hat{\mathbb{V}}, \mathbb{F})$ is a manageable braided multiplicative unitary on $\mathcal{L}$ relative to $\mathbb{W}$.

The proof will occupy the rest of this section. For $(\mathbb{U}, \hat{\mathbb{V}}, \mathbb{F})$ to be a braided multiplicative unitary, it only remains to verify the braided pentagon equation, which is equivalent to (4.4). We shall use the properties of the quantum exponential function established in [30].

The operators

$$
R:=X_{12}=\Upsilon q^{-2 \hat{\mathcal{N}}} \otimes \Upsilon^{-1} \otimes 1, \quad S:=X_{12} X_{23}=\Upsilon q^{-2 \hat{\mathcal{N}}} \otimes q^{-2 \hat{\mathcal{N}}} \otimes \Upsilon^{-1}
$$

are normal and satisfy the commutation relations in [30, (0.1)], that is, their phases and their absolute values strongly commute and

$$
\Phi_{R}^{*}|S| \Phi_{R}=q|S|, \quad \Phi_{S}|R| \Phi_{S}^{*}=q|R| .
$$

Since $R^{-1} S=X_{23}$ is also normal with spectrum $\overline{\mathbb{C}}_{q}$, [30, Theorems 2.1-2] apply and show that $R+S$ is normal with spectrum $\overline{\mathbb{C}}_{q}$ and

$$
\mathrm{F}_{q}\left(X_{23}\right) \cdot X_{12} \cdot \mathrm{F}_{q}\left(X_{23}\right)^{*}=\mathrm{F}_{q}\left(R^{-1} S\right) \cdot R \cdot \mathrm{F}_{q}\left(R^{-1} S\right)^{*}=R \dot{+} S .
$$

Moreover, 30, Theorem 3.1] gives

$$
\mathrm{F}_{q}(R) \mathrm{F}_{q}(S)=\mathrm{F}_{q}(R+S) .
$$

Both results of [30] together give (4.5) for $F=\mathrm{F}_{q}$; this is equivalent to the braided pentagon equation for $\mathbb{F}$.

Now we turn to braided manageability. First we compute the unitary $\widetilde{Z}$. It is the unique unitary on $\overline{\mathcal{L}} \otimes \mathcal{L}$ that satisfies (3.20). The contragradient $\widetilde{U}^{*}$ of $\mathrm{U}$ is given in the standard basis $\overline{e_{k, l}} \otimes e_{m}$ of $\overline{\mathcal{L}} \otimes \mathcal{H}$ by $\widetilde{\mathrm{U}}^{*}\left(\overline{e_{k, l}} \otimes e_{m}\right)=\overline{e_{k, l}} \otimes e_{m-l}$. Hence $\widetilde{Z} \in \mathcal{U}(\overline{\mathcal{L}} \otimes \mathcal{L})$ acts on the standard basis by

$$
\widetilde{Z}\left(\overline{e_{k, l}} \otimes e_{n, p}\right)=\overline{e_{k, l}} \otimes e_{n-l, p} .
$$

Equivalently, $\widetilde{Z}=(1 \otimes \mathcal{U})^{\hat{\mathcal{N}}^{\top} \otimes 1}$.

Next we define the operator $Q_{\mathcal{L}}$ required by Definition 3.15 .

$$
Q_{\mathcal{L}} e_{k, l}:=q^{-l} e_{k, l} \text {. }
$$

This is a strictly positive operator on $\mathcal{L}$ with spectrum $q^{\mathbb{Z}} \cup\{0\}$. It commutes with $\mathcal{U}$ and $\hat{\mathcal{N}}$ and therefore satisfies (3.16) and (3.17). The operator $Q_{\mathcal{L}} \otimes Q_{\mathcal{L}}$, mapping $e_{k, l} \otimes e_{n, p} \mapsto q^{-(l+p)} e_{k, l} \otimes e_{n, p}$, commutes with $X=\Upsilon q^{-2 \hat{\mathcal{N}}} \otimes \Upsilon^{-1}$ and therefore with $\mathbb{F}=\mathrm{F}_{q}(X)$. Thus (3.18) holds as well.

Finally, we need a unitary $\widetilde{\mathbb{F}} \in \mathcal{U}(\overline{\mathcal{L}} \otimes \mathcal{L})$ that satisfies 3.19). It suffices to check this if the vectors $x, y, u, v$ involved are standard basis vectors $x=e_{k, l}$, 
$y=e_{n, p}, u=e_{a, b}, v=e_{c, d}$ for $\mathcal{L}$. Using our explicit formulas for $Z$ and $\widetilde{Z}$, we may rewrite (3.19) as

$$
\left(e_{k, l} \otimes e_{a-l, b}|\mathbb{F}| e_{n, p} \otimes e_{c, d}\right)=\left(\overline{e_{n, p}} \otimes q^{-b} e_{a, b}|\widetilde{\mathbb{F}}| \overline{e_{k, l}} \otimes q^{d} e_{c+l, d}\right) .
$$

Substituting $\gamma=c+l$ and $\mathbb{F}=\mathrm{F}_{q}(X)$, this becomes

$$
\left(\overline{e_{n, p}} \otimes e_{a, b}|\widetilde{\mathbb{F}}| \overline{e_{k, l}} \otimes e_{\gamma, d}\right)=q^{b-d}\left(e_{k, l} \otimes e_{a-l, b}\left|\mathrm{~F}_{q}(X)\right| e_{n, p} \otimes e_{\gamma-l, d}\right)
$$

for $a, b, \gamma, d, k, l, n, p \in \mathbb{Z}$. So the issue is whether the bilinear form $\widetilde{\mathbb{F}}$ defined by this equation is unitary.

To compute the right hand side in (4.8), we Fourier transform the restrictions of $\mathrm{F}_{q}$ to the circles $|z|=q^{n}, n \in \mathbb{Z}$, and write

$$
\mathrm{F}_{q}(z)=\sum_{m \in \mathbb{Z}} \mathrm{F}_{m}(|z|) \Phi_{z}^{m}
$$

see [1] or [29, Appendix A]. The scalars $\mathrm{F}_{m}\left(q^{n}\right)$ for $m, n \in \mathbb{Z}$ are real and satisfy

$$
\mathrm{F}_{m}\left(q^{n}\right)=(-q)^{m} \mathrm{~F}_{-m}\left(q^{n-m}\right) .
$$

The vectors $e_{n, p} \otimes e_{\gamma-l, d}$ and $e_{k, l} \otimes e_{a-l, b}$ are eigenvectors of $|X|$ with eigenvalues $q^{2(n-\gamma+l)-(p+d)+1}$ and $q^{2(k-a+l)-(b+l)+1}$, respectively. And $\Phi_{X}^{m}$ acts on these vectors by $e_{k, l} \otimes e_{a, b} \mapsto e_{k, l+m} \otimes e_{a, b-m}$. Thus

$$
\begin{aligned}
& q^{b-d}\left(e_{k, l} \otimes e_{a-l, b}\left|\mathrm{~F}_{q}(X)\right| e_{n, p} \otimes e_{\gamma-l, d}\right) \\
= & \sum_{m \in \mathbb{Z}} q^{b-d}\left(e_{k, l} \otimes e_{a-l, b}\left|\Phi_{X}^{m} \mathrm{~F}_{m}(|X|)\right| e_{n, p} \otimes e_{\gamma-l, d}\right) \\
= & \sum_{m \in \mathbb{Z}} q^{b-d} \cdot \mathrm{F}_{m}\left(q^{2(n-\gamma+l)-(p+d)+1}\right) \delta_{k, n} \delta_{l, p+m} \delta_{a-l, \gamma-l} \delta_{b, d-m} \\
= & \delta_{k, n} \delta_{a, \gamma} \delta_{p, l+b-d} \cdot q^{b-d} \cdot \mathrm{F}_{d-b}\left(q^{2 k-2 a+l-b+1}\right) \\
= & \delta_{k, n} \delta_{a, \gamma} \delta_{p, l+b-d} \cdot(-1)^{b-d} \cdot \mathrm{F}_{b-d}\left(q^{2 k-2 a+l-d+1}\right) .
\end{aligned}
$$

Now we define an unbounded normal operator $\widetilde{X}$ on $\overline{\mathcal{L}} \otimes \mathcal{H}$ with spectrum $\overline{\mathbb{C}}_{q}$ by

$$
|\widetilde{X}|\left(\overline{e_{k, l}} \otimes e_{n, p}\right)=q^{2(k-n)+l-p+1} \overline{e_{k, l}} \otimes e_{n, p}, \quad \Phi_{\widetilde{X}}\left(\overline{e_{k, l}} \otimes e_{n, p}\right)=-\overline{e_{k, l+1}} \otimes e_{n, p+1},
$$

so $\widetilde{X}\left(\overline{e_{k, l}} \otimes e_{n, p}\right)=-1 \cdot q^{2(k-n)+l-p+1} \overline{e_{k, l+1}} \otimes e_{n, p+1}$. We claim that the unitary $\widetilde{\mathbb{F}}:=\mathrm{F}_{q}(\widetilde{X})^{*}$ will do:

$$
\begin{aligned}
\left(\overline{e_{n, p}} \otimes e_{a, b}|\widetilde{\mathbb{F}}| \overline{e_{k, l}} \otimes e_{\gamma, d}\right) & =\sum_{m \in \mathbb{Z}}\left(\overline{e_{k, l}} \otimes e_{\gamma, d}\left|\Phi_{\widetilde{X}}^{m} \mathrm{~F}_{m}(|\widetilde{X}|)\right| \overline{e_{n, p}} \otimes e_{a, b}\right) \\
& =\sum_{m \in \mathbb{Z}}(-1)^{m} \mathrm{~F}_{m}\left(q^{2(n-a)+p-b+1}\right) \delta_{k, n} \delta_{l, p+m} \delta_{\gamma, a} \delta_{d, b+m} \\
& =\delta_{k, n} \delta_{a, \gamma} \delta_{p, l+b-d} \cdot(-1)^{d-b} \cdot \mathrm{F}_{d-b}\left(q^{2 k-2 a+l-d+1}\right) .
\end{aligned}
$$

This is equal to the result of the computation above, so (4.8) holds. Thus our braided multiplicative unitary is manageable, and Theorem 4.7 is proved.

\section{Appendix A. Some Manageability Techniques}

Let $\mathbb{W}^{A} \in \mathcal{U}\left(\mathcal{H}_{A} \otimes \mathcal{H}_{A}\right)$ and $\mathbb{W}^{B} \in \mathcal{U}\left(\mathcal{H}_{B} \otimes \mathcal{H}_{B}\right)$ be manageable multiplicative unitaries as in [28, Definition 1.2], which generate $\mathrm{C}^{*}$-quantum groups $\mathbb{G}=\left(A, \Delta_{A}\right)$ and $\mathbb{H}=\left(B, \Delta_{B}\right)$.

Let $\mathrm{V} \in \mathcal{U}(\hat{A} \otimes B)$ be a bicharacter from $\mathbb{G}$ to $\mathbb{H}$. Let $\mathbb{V} \in \mathcal{U}\left(\mathcal{H}_{A} \otimes \mathcal{H}_{B}\right)$ be the concrete realisation of $\mathrm{V}$. Then $\mathbb{V} \in \mathcal{U}\left(\mathcal{H}_{A} \otimes \mathcal{H}_{B}\right)$ is adapted to $\mathbb{W}^{B}$ in the sense of 
28. Definition 1.3] by [10, Lemma 3.2]. Thus $\mathbb{V} \in \mathcal{U}\left(\mathcal{H}_{A} \otimes \mathcal{H}_{B}\right)$ is manageable by [28, Theorem 1.6]; that is, there is a unitary $\widetilde{\mathbb{V}} \in \mathcal{U}\left(\overline{\mathcal{H}}_{A} \otimes \mathcal{H}_{B}\right)$ with

$$
(x \otimes u|\mathbb{V}| z \otimes y)=\left(\bar{z} \otimes Q_{B} u|\widetilde{\mathbb{V}}| \bar{x} \otimes Q_{B}^{-1} y\right)
$$

for all $x, z \in \mathcal{H}_{A}, u \in \mathcal{D}\left(Q_{B}\right)$ and $y \in \mathcal{D}\left(Q_{B}^{-1}\right)$; here $Q_{B}$ is one of the operators in the manageability condition for $\mathbb{W}^{B}$.

Lemma A.2. $\mathbb{V}\left(Q_{A} \otimes Q_{B}\right) \mathbb{V}^{*}=Q_{A} \otimes Q_{B}$ and $\widetilde{\mathbb{V}}\left(Q_{A}^{\top} \otimes Q_{B}^{-1}\right) \widetilde{\mathbb{V}}^{*}=Q_{A}^{\top} \otimes Q_{B}^{-1}$.

Proof. The scaling group $\tau_{t}^{A}: \mathbb{R} \rightarrow \operatorname{Aut}(A)$ of $A$ acts through conjugation by $Q_{A}^{\text {it }}$ by [28, Theorem 1.5.5], and similarly for $B$. [10, (20)] says that $V$ is fixed by $\tau_{t}^{A} \otimes \tau_{t}^{B}$. This means that $\mathbb{V}$ commutes with $Q_{A} \otimes Q_{B}$, as asserted.

Hence the left-hand side of (A.1) does not change if we replace $x, u, z$ and $y$ by $Q_{A}^{\mathrm{it}}(x), Q_{B}^{\mathrm{it}}(u), Q_{A}^{\mathrm{it}}(z)$ and $Q_{B}^{\mathrm{it}}(y)$, respectively, for any $t \in \mathbb{R}$. Thus the right-hand sides also remain the same, that is,

$$
\left(\bar{z} \otimes Q_{B} u|\widetilde{\mathbb{V}}| \bar{x} \otimes Q_{B}^{-1} y\right)=\left(\left[Q_{A}^{\top}\right]^{-\mathrm{i} t} \bar{z} \otimes Q_{B}^{\mathrm{i} t} Q_{B} u|\widetilde{\mathbb{V}}|\left[Q_{A}^{\top}\right]^{-\mathrm{i} t} \bar{x} \otimes Q_{B}^{\mathrm{i} t} Q_{B}^{-1} u\right) .
$$

Hence $\widetilde{\mathbb{V}}=\left(\left[Q_{A}^{\top}\right]^{\mathrm{i} t} \otimes Q_{B}^{-\mathrm{i} t}\right) \widetilde{\mathbb{V}}\left(\left[Q_{A}^{\top}\right]^{\mathrm{i} t} \otimes Q_{B}^{-\mathrm{i} t}\right)$ for all $t \in \mathbb{R}$. This says that $\widetilde{\mathbb{V}}$ commutes with $Q_{A}^{\top} \otimes Q_{B}^{-1}$.

Lemma A.3. Let $Q$ be a self-adjoint, strictly positive operator on a Hilbert space $\mathcal{H}$. There is an orthonormal basis $\left(e_{i}\right)_{i \in \mathbb{N}}$ in $\mathcal{H}$ with $e_{i} \in \mathcal{D}(Q) \cap \mathcal{D}\left(Q^{-1}\right)$ and strong convergence

$$
\sum_{i \in \mathbb{N}}\left|Q^{-1} e_{i}\right\rangle\left\langle Q e_{i}\right|=\operatorname{id}_{\mathcal{H}} .
$$

Proof. For $n \in \mathbb{Z}$ and $\lambda \in\left[2^{2 n-1}, 2^{2 n+1}\right)$, let $f(\lambda)=2^{-2 n} \lambda$ and $g(\lambda)=\lambda / f(\lambda)$. The function

$$
\mathbb{R}_{>0} \ni \lambda \mapsto f(\lambda) \in\left[2^{-1}, 2\right)
$$

is piecewise linear and bounded with bounded inverse. Hence the Borel functional calculus for self-adjoint operators gives $Q^{\prime}:=f(Q)$, which is self-adjoint and bounded with a bounded inverse. We also get the self-adjoint operator $Q^{\prime \prime}=g(Q)$, which has countable spectrum $\left\{2^{2 n} \mid n \in \mathbb{Z}\right\}$. Thus $\mathcal{H}$ is the orthogonal direct sum of the $2^{2 n}$-eigenspaces of $\mathcal{H}$. We choose orthonormal bases for all these eigenspaces and put them together to an orthonormal basis $\left(e_{i}\right)_{i \in \mathbb{N}}$ of $\mathcal{H}$. We have $Q=Q^{\prime} Q^{\prime \prime}$ and $Q^{-1}=\left(Q^{\prime}\right)^{-1}\left(Q^{\prime \prime}\right)^{-1}$ by functional calculus. Since the operators $\left(Q^{\prime}\right)^{ \pm 1}$ are bounded and self-adjoint, $Q$ and $Q^{\prime \prime}$ have the same domain. Thus $e_{i} \in \mathcal{D}(Q) \cap \mathcal{D}\left(Q^{-1}\right)$ because it is an eigenvector of $Q^{\prime \prime}$ with some positive eigenvalue $2^{2 n}$ for some $n \in \mathbb{Z}$ depending on $i$. Since $\left(Q^{\prime}\right)^{ \pm 1}$ are bounded and $Q^{\prime \prime} e_{i}=2^{2 n} e_{i}$, we may rewrite

$$
\begin{aligned}
\left|Q^{-1} e_{i}\right\rangle\left\langle Q e_{i}|=|\left(Q^{\prime}\right)^{-1}\left(Q^{\prime \prime}\right)^{-1} e_{i}\right\rangle\left\langle Q^{\prime} Q^{\prime \prime} e_{i}|=|\left(Q^{\prime}\right)^{-1} 2^{-2 n} e_{i}\right\rangle\left\langle Q^{\prime} 2^{2 n} e_{i}\right| \\
=\left(Q^{\prime}\right)^{-1}\left|e_{i}\right\rangle\left\langle e_{i}\right| Q^{\prime} .
\end{aligned}
$$

The sum $\sum_{i \in \mathbb{N}}\left|e_{i}\right\rangle\left\langle e_{i}\right|$ converges strongly to the identity on $\mathcal{H}$ because $\left(e_{i}\right)_{i \in \mathbb{N}}$ is an orthonormal basis for $\mathcal{H}$. Since $\left(Q^{\prime}\right)^{ \pm 1}$ are bounded, the sum over $\left(Q^{\prime}\right)^{-1}\left|e_{i}\right\rangle\left\langle e_{i}\right| Q^{\prime}$ converges strongly to $\left(Q^{\prime}\right)^{-1} \cdot 1 \cdot Q^{\prime}=1$.

The following lemma completes the proof of Theorem 3.29

Lemma A.5. In the situation of the proof of Theorem $3.29, \mathbb{W}^{C}, Q$ and $\widetilde{\mathbb{W}} C$ verify (3.14). 
Proof. We continue in the notation of the proof of Theorem 3.29 It suffices to check (3.14) when $x, z, u, y$ are tensor monomials: $x=x_{1} \otimes x_{2}, z=z_{1} \otimes z_{2}$, $u=u_{1} \otimes u_{2}, y=y_{1} \otimes y_{2}$ with $x_{1}, z_{1} \in \mathcal{H}, x_{2}, z_{2} \in \mathcal{L}, u_{1} \in \mathcal{D}(Q), u_{2} \in \mathcal{D}\left(Q_{\mathcal{L}}\right)$, $y_{1} \in \mathcal{D}\left(Q^{-1}\right), y_{2} \in \mathcal{D}\left(Q_{\mathcal{L}}^{-1}\right)$. This implies the assertion for all $x, y, z, u$.

Equation (3.10) is equivalent to $\mathbb{U}_{23} \hat{\mathbb{V}}_{34}^{*}=\hat{\mathbb{V}}_{34}^{*} \mathbb{U}_{23} Z_{13}^{*}$, which gives

$$
\mathbb{W}_{1234}^{C}=\mathbb{W}_{13} \hat{\mathbb{V}}_{34}^{*} \mathbb{U}_{23}\left(Z_{24}^{*} \mathbb{F}_{24}\right) \hat{\mathbb{V}}_{34} .
$$

We first concentrate on the part $\mathbb{U}_{23}\left(Z_{24}^{*} \mathbb{F}_{24}\right)$ in (A.6). Let $\left(e_{i}\right)_{i \in \mathbb{N}}$ be an orthonormal basis of $\mathcal{L}$. Then (3.30) and (3.19) give

$$
\begin{aligned}
& \left\langle x_{2} \otimes u_{1} \otimes u_{2}\left|\mathbb{U}_{12} Z_{13}^{*} \mathbb{F}_{13}\right| z_{2} \otimes y_{1} \otimes y_{2}\right\rangle \\
= & \sum\left\langle x_{2} \otimes u_{1} \otimes u_{2}\left|\mathbb{U}_{12} \cdot\left(\left|e_{i}\right\rangle\left\langle e_{i}\right| \otimes 1 \otimes 1\right) \cdot Z_{13}^{*} \mathbb{F}_{13}\right| z_{2} \otimes y_{1} \otimes y_{2}\right\rangle \\
= & \sum\left\langle x_{2} \otimes u_{1}|\mathbb{U}| e_{i} \otimes y_{1}\right\rangle \cdot\left\langle e_{i} \otimes u_{2}\left|Z^{*} \mathbb{F}\right| z_{2} \otimes y_{2}\right\rangle \\
= & \sum\left\langle\overline{z_{2}} \otimes Q_{\mathcal{L}}\left(u_{2}\right)|\widetilde{\mathbb{F}}| \overline{e_{i}} \otimes Q_{\mathcal{L}}^{-1}\left(y_{2}\right)\right\rangle\left\langle\overline{e_{i}} \otimes Q\left(u_{1}\right)|\widetilde{\mathbb{U}}| \overline{x_{2}} \otimes Q^{-1}\left(y_{1}\right)\right\rangle \\
= & \left\langle\overline{z_{2}} \otimes Q\left(u_{1}\right) \otimes Q_{\mathcal{L}}\left(u_{2}\right)\left|\widetilde{\mathbb{F}}_{13} \widetilde{\mathbb{U}}_{12}\right| \overline{x_{2}} \otimes Q^{-1}\left(y_{1}\right) \otimes Q_{\mathcal{L}}^{-1}\left(y_{2}\right)\right\rangle
\end{aligned}
$$

Since $\hat{\mathbb{V}}$ commutes with $Q \otimes Q_{\mathcal{L}}$, it also preserves the domains of $\left(Q \otimes Q_{\mathcal{L}}\right)^{-1}$, and we get an equivalent statement if we replace $u_{1} \otimes u_{2}$ and $y_{1} \otimes y_{2}$ above by $\hat{\mathbb{V}}\left(u_{1} \otimes u_{2}\right)$ and $\hat{\mathbb{V}}\left(y_{1} \otimes y_{2}\right)$, respectively. This gives

$$
\begin{aligned}
\left\langle x_{2}\right. & \left.\otimes u_{1} \otimes u_{2}\left|\hat{\mathbb{V}}_{23}^{*} \mathbb{U}_{12} Z_{13}^{*} \mathbb{F}_{13} \hat{\mathbb{V}}_{23}\right| z_{2} \otimes y_{1} \otimes y_{2}\right\rangle \\
& =\left\langle\overline{z_{2}} \otimes Q\left(u_{1}\right) \otimes Q_{\mathcal{L}}\left(u_{2}\right)\left|\hat{\mathbb{V}}_{23}^{*} \widetilde{\mathbb{F}}_{13} \widetilde{\mathbb{U}}_{12} \hat{\mathbb{V}}_{23}\right| \overline{x_{2}} \otimes Q^{-1}\left(y_{1}\right) \otimes Q_{\mathcal{L}}^{-1}\left(y_{2}\right)\right\rangle .
\end{aligned}
$$

Now let $\left(\epsilon_{j}\right)_{j \in \mathbb{N}}$ be a basis of $\mathcal{H}$ as in Lemma A.3, that is,

$$
\sum_{j}\left|Q^{-1}\left(\epsilon_{j}\right)\right\rangle\left\langle Q\left(\epsilon_{j}\right)\right|=\operatorname{id}_{\mathcal{H}} .
$$

We compute

$$
\begin{aligned}
&\left\langle x_{1} \otimes x_{2} \otimes u_{1} \otimes u_{2}\left|\mathbb{W}_{13} \hat{\mathbb{V}}_{34}^{*} \mathbb{U}_{23} Z_{24}^{*} \mathbb{F}_{24} \hat{\mathbb{V}}_{34}\right| z_{1} \otimes z_{2} \otimes y_{1} \otimes y_{2}\right\rangle \\
&= \sum_{j}\left\langle x_{1} \otimes x_{2} \otimes u_{1} \otimes u_{2}\left|\mathbb{W}_{13} \cdot\left(1 \otimes 1 \otimes|| \epsilon_{j}\right\rangle\left\langle\epsilon_{j}\right| \otimes 1\right) \cdot \hat{\mathbb{V}}_{34}^{*} \mathbb{U}_{23} Z_{24}^{*} \mathbb{F}_{24} \hat{\mathbb{V}}_{34}\right| \\
&= \sum_{j}\left\langle x_{1} \otimes u_{1}|\mathbb{W}| z_{1} \otimes z_{2} \otimes \epsilon_{1} \otimes \cdot\left\langle x_{2} \otimes \epsilon_{i} \otimes u_{2}\left|\hat{\mathbb{V}}_{23}^{*} \mathbb{U}_{12}\left(Z^{*} \mathbb{F}\right)_{13} \hat{\mathbb{V}}_{23}\right| z_{2} \otimes y_{1} \otimes y_{2}\right\rangle\right. \\
&= \sum_{j}\left\langle\overline{z_{1}} \otimes Q\left(u_{1}\right)|\widetilde{\mathbb{W}}| \overline{x_{1}} \otimes Q^{-1}\left(\epsilon_{i}\right)\right\rangle \\
& \cdot \quad\left\langle\overline{z_{2}} \otimes Q\left(\epsilon_{i}\right) \otimes Q_{\mathcal{L}}\left(u_{2}\right)\left|\hat{\mathbb{V}}_{23}^{*} \widetilde{\mathbb{F}}_{13} \widetilde{\mathbb{U}}_{12} \hat{\mathbb{V}}_{23}\right| \overline{x_{2}} \otimes Q^{-1}\left(y_{1}\right) \otimes Q_{\mathcal{L}}^{-1}\left(y_{2}\right)\right\rangle \\
&=\left\langle\overline{z_{1}} \otimes \overline{z_{2}} \otimes Q\left(u_{1}\right) \otimes Q_{\mathcal{L}}\left(u_{2}\right)\left|\widetilde{\mathbb{W}}_{13} \hat{\mathbb{V}}_{34}^{*} \widetilde{\mathbb{F}}_{24} \widetilde{\mathbb{U}}_{23} \hat{\mathbb{V}}_{34}\right|\right. \\
&
\end{aligned}
$$

Thus (3.14) holds for $\mathbb{W}^{C}, Q$ and $\widetilde{\mathbb{W}}^{C}$.

Lemma A.8. The unitary $\widetilde{\mathbb{F}} \in \mathcal{U}(\overline{\mathcal{L}} \otimes \mathcal{L})$ defined by (3.46) satisfies the manageability condition (3.19) for $\mathbb{F} \in \mathcal{U}(\mathcal{L} \otimes \mathcal{L})$ in Theorem 3.37 . 
Proof. It suffices to check (3.19) when $x, u, y, v$ are tensor monomials: $x=\bar{x}_{1} \otimes x_{2}$, $u=\bar{u}_{1} \otimes u_{2}, y=\bar{y}_{1} \otimes y_{2}, v=\bar{v}_{1} \otimes v_{2}$, with $x_{1}, x_{2}, y_{1}, y_{2} \in \mathcal{H}, v_{1}, u_{2} \in \mathcal{D}\left(Q_{C}\right)$ and $u_{1}, v_{2} \in \mathcal{D}\left(Q_{C}^{-1}\right)$.

First we focus on the part $\Sigma_{23} \widetilde{\widehat{\mathbb{P}}}_{23} \Sigma_{23} \mathbb{P}_{13}^{\top \otimes \top}$. Let $\left(e_{i}\right)_{i \in \mathbb{N}}$ be an orthonormal basis of $\mathcal{H}$ in Lemma A.3. Then $\left(\bar{e}_{i}\right)_{i \in \mathbb{N}}$ is an orthonormal basis of $\overline{\mathcal{H}}$ with

$$
\sum_{i \in \mathbb{N}}\left|\overline{Q e_{i}}\right\rangle\left\langle\overline{Q^{-1} e_{i}}\right|=\mathrm{id}_{\overline{\mathcal{H}}}
$$

Equation (3.14) for $\mathbb{P}$ and $\widehat{\mathbb{P}}$ gives

$$
\begin{aligned}
& \left\langle\bar{x}_{1} \otimes x_{2} \otimes \bar{u}_{1}\left|\Sigma_{23} \widetilde{\widehat{\mathbb{P}}}_{23} \Sigma_{23} \mathbb{P}_{13}^{\top \otimes \top}\right| \bar{y}_{1} \otimes y_{2} \otimes \bar{v}_{1}\right\rangle \\
& =\sum\left\langle\bar{x}_{1} \otimes x_{2} \otimes \bar{u}_{1}\left|\Sigma_{23} \widetilde{\widehat{\mathbb{P}}}_{23} \Sigma_{23} \cdot\left(1 \otimes 1 \otimes\left|e_{i}\right\rangle\left\langle e_{i}\right|\right) \cdot \mathbb{P}_{13}^{\mathbf{T} \otimes \top}\right| \bar{y}_{1} \otimes y_{2} \otimes \bar{v}_{1}\right\rangle \\
& =\sum\left\langle x_{2} \otimes \bar{u}_{1}|\Sigma \widetilde{\widehat{\mathbb{P}}} \Sigma| y_{2} \otimes \bar{e}_{i}\right\rangle\left\langle\bar{x}_{1} \otimes \bar{e}_{i}\left|\mathbb{P}^{\mathbf{T} \otimes \mathbf{T}}\right| \bar{y}_{1} \otimes \bar{v}_{1}\right\rangle \\
& =\sum\left\langle\bar{u}_{1} \otimes x_{2}|\widetilde{\widehat{\mathbb{P}}}| \bar{e}_{i} \otimes y_{2}\right\rangle\left\langle y_{1} \otimes v_{1}|\mathbb{P}| x_{1} \otimes e_{i}\right\rangle \\
& =\sum\left\langle e_{i} \otimes Q_{C}^{-1}\left(x_{2}\right)|\widehat{\mathbb{P}}| u_{1} \otimes Q_{C}\left(y_{2}\right)\right\rangle\left\langle\bar{x}_{1} \otimes Q_{C}\left(v_{1}\right)|\widetilde{\mathbb{P}}| \bar{y}_{1} \otimes Q_{C}^{-1}\left(e_{i}\right)\right\rangle \text {. }
\end{aligned}
$$

Lemma A.2 shows that $Q_{C} \otimes C_{C}$ commutes with $\widehat{\mathbb{P}}$. Hence

$$
\begin{aligned}
& \left\langle\bar{x}_{1} \otimes x_{2} \otimes \bar{u}_{1}\left|\Sigma_{23} \widetilde{\widehat{\mathbb{P}}}_{23} \Sigma_{23} \mathbb{P}_{13}^{\mathrm{\top} \otimes \mathrm{T}}\right| \bar{y}_{1} \otimes y_{2} \otimes \bar{v}_{1}\right\rangle \\
& =\sum\left\langle Q_{C}\left(e_{i}\right) \otimes x_{2}|\widehat{\mathbb{P}}| Q_{C}^{-1}\left(u_{1}\right) \otimes y_{2}\right\rangle\left\langle\bar{x}_{1} \otimes Q_{C}\left(v_{1}\right)|\widetilde{\mathbb{P}}| \bar{y}_{1} \otimes Q_{C}^{-1}\left(e_{i}\right)\right\rangle \\
& =\sum\left\langle x_{2} \otimes Q_{C}\left(e_{i}\right)\left|\mathbb{P}^{*}\right| y_{2} \otimes Q_{C}^{-1}\left(u_{1}\right)\right\rangle\left\langle y_{1} \otimes \overline{Q_{C}^{-1}\left(e_{i}\right)}\left|\widetilde{\mathbb{P}}^{\mathbf{\top} \otimes \mathrm{T}}\right| x_{1} \otimes \overline{Q_{C}\left(v_{1}\right)}\right\rangle \\
& =\sum\left\langle\bar{y}_{2} \otimes \overline{Q_{C}^{-1}\left(u_{1}\right)}\left|\left(\mathbb{P}^{*}\right)^{\mathrm{T} \otimes \mathrm{T}}\right| \bar{x}_{2} \otimes \overline{Q_{C}\left(e_{i}\right)}\right\rangle\left\langle y_{1} \otimes \overline{Q_{C}^{-1}\left(e_{i}\right)}\left|\widetilde{\mathbb{P}}^{\mathrm{T} \otimes \mathrm{T}}\right| x_{1} \otimes \overline{Q_{C}\left(v_{1}\right)}\right\rangle .
\end{aligned}
$$

Now A.9 gives

$$
\begin{aligned}
& \left\langle\bar{x}_{1} \otimes x_{2} \otimes \bar{u}_{1}\left|\Sigma_{23} \widetilde{\widehat{\mathbb{P}}}_{23} \Sigma_{23} \mathbb{P}_{13}^{\top \otimes \top}\right| \bar{y}_{1} \otimes y_{2} \otimes \bar{v}_{1}\right\rangle \\
& =\left\langle y_{1} \otimes \bar{y}_{2} \otimes \overline{Q_{C}^{-1}\left(u_{1}\right)}\left|\left(\mathbb{P}^{*}\right)_{23}^{\mathrm{T} \otimes \mathrm{T}} \widetilde{\mathbb{P}}_{13}^{\mathrm{\top} \otimes \mathrm{T}}\right| x_{1} \otimes \bar{x}_{2} \otimes \overline{Q_{C}\left(v_{1}\right)}\right\rangle .
\end{aligned}
$$

A similar computation gives

$$
\begin{aligned}
\left\langle\bar{x}_{1} \otimes x_{2} \otimes u_{2}\left|\mathbb{W}_{23}^{C}\left(\widetilde{\mathbb{W}}_{13}^{C}\right)^{*}\right| \bar{y}_{1} \otimes y_{2} \otimes v_{2}\right\rangle & \\
& =\left\langle y_{1} \otimes \bar{y}_{2} \otimes Q_{C}\left(u_{2}\right)\left|\widetilde{\mathbb{W}}_{23}^{C}\left(\mathbb{W}_{13}^{C}\right)^{*}\right| x_{1} \otimes \bar{x}_{2} \otimes Q_{C}^{-1}\left(v_{2}\right)\right\rangle .
\end{aligned}
$$


Let $\left(e_{j}\right)_{j \in \mathbb{N}}$ be an orthonormal basis of $\mathcal{H}$. Equations A.10 and A.11 imply

$$
\begin{aligned}
& \left\langle\bar{x}_{1} \otimes x_{2} \otimes \bar{u}_{1} \otimes u_{2}\left|\Sigma_{23} \widetilde{\widehat{P}}_{23} \Sigma_{23} \mathbb{P}_{13}^{\top \otimes \top} \mathbb{W}_{24}^{C}\left(\widetilde{\mathbb{W}}_{14}^{C}\right)^{*}\right| \bar{y}_{1} \otimes y_{2} \otimes \bar{v}_{1} \otimes v_{2}\right\rangle \\
& =\sum_{j, k}\left\langle\bar{x}_{1} \otimes x_{2} \otimes \bar{u}_{1} \otimes u_{2}\right| \Sigma_{23} \widetilde{\widehat{\mathbb{P}}}_{23} \Sigma_{23} \mathbb{P}_{13}^{\mathrm{T} \otimes \mathrm{T}} \cdot\left(\left|\bar{e}_{j}\right\rangle\left\langle\bar{e}_{j}|\otimes| e_{k}\right\rangle\left\langle e_{k}\right| \otimes 1 \otimes 1\right) \\
& \cdot \mathbb{W}_{24}^{C}\left(\widetilde{\mathbb{W}}_{14}^{C}\right)^{*}\left|\bar{y}_{1} \otimes y_{2} \otimes \bar{v}_{1} \otimes v_{2}\right\rangle \\
& =\sum_{j, k}\left\langle\bar{x}_{1} \otimes x_{2} \otimes \bar{u}_{1}\left|\Sigma_{23} \widetilde{\widetilde{P}}_{23} \Sigma_{23} \mathbb{P}_{13}^{\mathbf{T} \otimes \mathbf{T}}\right| \bar{e}_{j} \otimes e_{k} \otimes \bar{v}_{1}\right\rangle \\
& \left\langle\bar{e}_{j} \otimes e_{k} \otimes u_{2}\left|\mathbb{W}_{23}^{C}\left(\widetilde{\mathbb{W}}_{13}^{C}\right)^{*}\right| \bar{y}_{1} \otimes y_{2} \otimes v_{2}\right\rangle \\
& =\sum_{j, k}\left\langle y_{1} \otimes \bar{y}_{2} \otimes Q_{C}\left(u_{2}\right)\left|\widetilde{\mathbb{W}}_{23}^{C}\left(\mathbb{W}_{13}^{C}\right)^{*}\right| e_{j} \otimes \bar{e}_{k} \otimes Q_{C}^{-1}\left(v_{2}\right)\right\rangle \\
& \left\langle e_{j} \otimes \bar{e}_{k} \otimes \overline{Q_{C}^{-1}\left(u_{1}\right)}\left|\left(\mathbb{P}^{*}\right)_{23}^{\mathrm{T} \otimes \mathrm{T}} \widetilde{\mathbb{P}}_{13}^{\mathrm{T} \otimes \mathrm{T}}\right| x_{1} \otimes \bar{x}_{2} \otimes \overline{Q_{C}\left(v_{1}\right)}\right\rangle \\
& =\left\langle y_{1} \otimes \bar{y}_{2} \otimes \overline{Q_{C}^{-1}\left(u_{1}\right)} \otimes Q_{C}\left(u_{2}\right)\left|\widetilde{\mathbb{W}}_{24}^{C}\left(\mathbb{W}_{14}^{C}\right)^{*}\left(\mathbb{P}^{*}\right)_{23}^{\mathbf{T} \otimes \mathbf{T}} \widetilde{\mathbb{P}}_{13}^{\boldsymbol{\top} \otimes \mathrm{T}}\right|\right. \\
& \left.x_{1} \otimes \bar{x}_{2} \otimes \overline{Q_{C}\left(v_{1}\right)} \otimes Q_{C}^{-1}\left(v_{2}\right)\right\rangle
\end{aligned}
$$

This is the equation we have to check.

\section{REFERENCES}

[1] Saad Baaj, Représentation régulière du groupe quantique $E_{\mu}(2)$ de Woronowicz, C. R. Acad. Sci. Paris Sér. I Math. 314 (1992), no. 13, 1021-1026, available at http://gallica.bnf.fr/ark:/12148/bpt6k58688425/f1025.item MR 1168528

[2] Alcides Buss, Generalized fixed point algebras for coactions of locally compact quantum groups, Doctoral Thesis, Univ. Münster, 2007.

[3] — Generalized fixed point algebras for coactions of locally compact quantum groups, Münster J. Math. 6 (2013), 295-341, available at http://nbn-resolving.de/urn:nbn:de:hbz:6-55309458343 MR 3148214

[4] Matthew Daws, Paweł Kasprzak, Adam G. Skalski, and Piotr Mikołaj Sołtan, Closed quantum subgroups of locally compact quantum groups, Adv. Math. 231 (2012), no. 6, 3473-3501, doi: 10.1016/j.aim.2012.09.002 MR 2980506

[5] Paweł Kasprzak, Ralf Meyer, Sutanu Roy, and Stanisław Lech Woronowicz, Braided quantum SU(2) groups, J. Noncommut. Geom. (2016), accepted. arXiv: 1411.3218.

[6] Paweł Kasprzak and Piotr Mikołaj Sołtan, Quantum groups with projection on von Neumann algebra level, J. Math. Anal. Appl. 427 (2015), no. 1, 289-306, doi: 10.1016/j.jmaa.2015.02.047. MR 3318200

[7] Q Quantum groups with projection and extensions of locally compact quantum groups (2014), eprint. arXiv: 1412.0821

[8] Shahn Majid, Algebras and Hopf algebras in braided categories, Advances in Hopf algebras (Chicago, IL, 1992), 1994, pp. 55-105. MR 1289422

[9] Ralf Meyer, Generalized fixed point algebras and square-integrable groups actions, J. Funct. Anal. 186 (2001), no. 1, 167-195, doi: 10.1006/jfan.2001.3795 |MR 1863296

[10] Ralf Meyer, Sutanu Roy, and Stanisław Lech Woronowicz, Homomorphisms of quantum groups, Münster J. Math. 5 (2012), 1-24, available at http://nbn-resolving.de/urn:nbn:de:hbz:6-88399662599. MR 3047623

[11] , Quantum group-twisted tensor products of $\mathrm{C}^{*}$-algebras, Internat. J. Math. 25 (2014), no. 2, 1450019, 37, doi: 10.1142/S0129167X14500190 MR 3189775

[12] _ Quantum group-twisted tensor products of $\mathrm{C}^{*}$-algebras II, J. Noncommut. Geom. (2016), accepted. arXiv: 1501.04432

[13] Ryszard Nest and Christian Voigt, Equivariant Poincaré duality for quantum group actions, J. Funct. Anal. 258 (2010), no. 5, 1466-1503, doi: 10.1016/j.jfa.2009.10.015 MR 2566309

[14] Chi-Keung Ng, Morphisms of multiplicative unitaries, J. Operator Theory $\mathbf{3 8}$ (1997), no. 2, 203-224, available at http://www.theta.ro/jot/archive/1997-038-002/1997-038-002-001.html. MR 1606928 
[15] David E. Radford, The structure of Hopf algebras with a projection, J. Algebra 92 (1985), no. 2, 322-347, doi: 10.1016/0021-8693(85)90124-3 MR $\mathbf{7 7 8 4 5 2}$

[16] Marc A. Rieffel, Integrable and proper actions on $C^{*}$-algebras, and square-integrable representations of groups, Expo. Math. 22 (2004), no. 1, 1-53, doi: 10.1016/S0723-0869(04)80002-1 MR 2166968

[17] Sutanu Roy, C*-Quantum groups with projection, Ph.D. Thesis, Georg-August Universität Göttingen, 2013, http://hdl.handle.net/11858/00-1735-0000-0022-5EF9-0

[18] _ The Drinfeld double for $C^{*}$-algebraic quantum groups, J. Operator Theory $\mathbf{7 4}$ (2015), no. 2, 485-515, doi: 10.7900/jot.2014sep04.2053 MR 3431941

[19] _ Braided $C^{*}$-quantum groups (2016), eprint. arXiv: 1601.00169

[20] Piotr Mikołaj Sołtan, New quantum "az + b” groups, Rev. Math. Phys. 17 (2005), no. 3, 313-364, doi: 10.1142/S0129055X05002339 MR 2144675

[21] Piotr Mikołaj Sołtan and Stanisław Lech Woronowicz, From multiplicative unitaries to quantum groups. II, J. Funct. Anal. 252 (2007), no. 1, 42-67, doi: 10.1016/j.jfa.2007.07.006 MR 2357350

[22] Piotr Stachura, Towards a topological (dual of) quantum $\kappa$-Poincaré group, Rep. Math. Phys. 57 (2006), no. 2, 233-256, doi: 10.1016/S0034-4877(06)80019-4 MR 2227008

[23] _ On the quantum 'ax $+b$ ' group, J. Geom. Phys. 73 (2013), 125-149, doi: 10.1016/j.geomphys.2013.05.006 MR 3090106

[24] Stefaan Vaes, A new approach to induction and imprimitivity results, J. Funct. Anal. 229 (2005), no. 2, 317-374, doi: 10.1016/j.jfa.2004.11.016 MR 2182592

[25] Stefaan Vaes and Leonid Vainerman, Extensions of locally compact quantum groups and the bicrossed product construction, Adv. Math. 175 (2003), no. 1, 1-101, doi: 10.1016/S0001-8708(02)00040-3 MR 1970242

[26] Stanisław Lech Woronowicz, Quantum E(2) group and its Pontryagin dual, Lett. Math. Phys. 23 (1991), no. 4, 251-263, doi: 10.1007/BF00398822 MR 1152695

[27] _ $C^{*}$-algebras generated by unbounded elements, Rev. Math. Phys. 7 (1995), no. 3, 481-521, doi: 10.1142/S0129055X95000207 MR 1326143

[28] _ From multiplicative unitaries to quantum groups, Internat. J. Math. 7 (1996), no. 1, 127-149, doi: 10.1142/S0129167X96000086 MR 1369908

[29] Luantum ' $a z+b^{\prime}$ ' group on complex plane, Internat. J. Math. 12 (2001), no. 4, 461-503, doi: 10.1142/S0129167X01000836 MR 1841400

[30] - Operator equalities related to the quantum E(2) group, Comm. Math. Phys. 144 (1992), no. 2, 417-428, available at http://projecteuclid.org/euclid.cmp/1104249324 MR 1152380

[31] Stanisław Lech Woronowicz and Stanisław Zakrzewski, Quantum 'ax $+b$ ' group, Rev. Math. Phys. 14 (2002), no. 7-8, 797-828, doi: 10.1142/S0129055X02001405 MR 1932667

E-mail address: rmeyer2@uni-goettingen.de

Mathematisches Institut, Georg-August Universität Göttingen, Bunsenstrasse 3-5, 37073 Göttingen, Germany

E-mail address: sutanu.roy@carleton.ca

School of Mathematics and Statistics, Carleton University, 1125 Colonel By Drive, K1S 5B6 Ottawa, Canada.

E-mail address: Stanislaw.Woronowicz@fuw.edu.pl

Instytut Matematyczny Polskiej Akademi Nauk, Ul. Śniadeckich 8, 00-656 Warszawa, Poland, and, Katedra Metod Matematycznych Fizyki, Wydzial Fizyki, Uniwersytet Warszawski, ul. Pasteura 5, 02-093 Warszawa, Poland 